\title{
Radiative process of two entangled uniformly accelerated atoms in a thermal bath: a possible case of anti-Unruh event
}

\author{
Subhajit Barman and Bibhas Ranjan Majhi \\ Department of Physics, Indian Institute of Technology Guwahati, \\ Guwahati 781039, Assam, India \\ E-mail: subhajit.b@iitg.ac.in, bibhas.majhi@iitg.ac.in
}

ABSTRACT: We study the radiative process of two entangled two-level atoms uniformly accelerated in a thermal bath, coupled to a massless scalar field. First, by using the positive frequency Wightman function from the Minkowski modes with a Rindler transformation we provide the transition probabilities for the transitions from maximally entangled symmetric and anti-symmetric Bell states to the collective excited or ground state in $(1+1)$ and $(1+3)$ dimensions. We observe a possible case of anti-Unruh-like event in these transition probabilities, though the $(1+1)$ and $(1+3)$ dimensional results are not completely equivalent. We infer that thermal bath plays a major role in the occurrence of the anti-Unruh-like effect, as it is also present in the transition probabilities corresponding to a single detector in this case. Second, we have considered the Green's functions in terms of the Rindler modes with the vacuum of Unruh modes for estimating the same. Here the anti-Unruh effect appears only for the transition from the anti-symmetric state to the collective excited or ground state. It is noticed that here the $(1+1)$ and $(1+3)$ dimensional results are equivalent, and for a single detector, we do not observe any anti-Unruh effect. This suggests that the entanglement between the states of the atoms is the main cause for the observed anti-Unruh effect in this case. In going through the investigation, we find that the transition probability for a single detector case is symmetric under the interchange between the thermal bath's temperature and the Unruh temperature for Rindler mode analysis; whereas this is not the case for Minkowski mode. We further comment on whether this observation may shed light on the analogy between an accelerated observer and a real thermal bath. An elaborate investigation for the classifications of our observed anti-Unruh effects, i.e., either weak or strong anti-Unruh effect, is also thoroughly demonstrated.

Keywords: Classical Theories of Gravity, Thermal Field Theory

ArXiv EPrint: 2101.08186 


\section{Contents}

1 Introduction 1

2 Radiative process of two entangled atoms: a model set-up 4

3 Thermal Wightman function corresponding to Minkowski mode $\quad 7$

$\begin{array}{lll}3.1 & \text { An observer in a thermal bath } & 7\end{array}$

$\begin{array}{lll}3.2 & \text { An accelerated observer in a thermal bath } & 8\end{array}$

4 Thermal Wightman function corresponding to Rindler modes $\quad 10$

$\begin{array}{lll}4.1 & (1+1) \text { dimensions } & 10\end{array}$

$\begin{array}{lll}4.2(1+3) \text { dimensions } & 12\end{array}$

5 Transition probability for accelerated atoms in thermal bath with respect to Minkowski modes 14

$\begin{array}{lll}5.1 & (1+1) \text { dimensions } & 15\end{array}$

$\begin{array}{lll}5.2(1+3) \text { dimensions } & 17\end{array}$

6 Transition probability for accelerated atoms with the Rindler modes in

$\begin{array}{ll}\text { a thermal background } & 19\end{array}$

$6.1(1+1)$ dimensions 21

$6.2(1+3)$ dimensions $\quad 22$

7 Detailed investigation of observed anti-Unruh(-like) phenomenon $\quad 24$

$\begin{array}{ll}7.1 \text { Anti-Unruh effect: the conditions } & 26\end{array}$

$\begin{array}{ll}\text { 7.1.1 Weak anti-Unruh effect } & 26\end{array}$

$\begin{array}{ll}7.1 .2 & \text { Strong anti-Unruh effect } \\ \text { Case } & 26\end{array}$

$\begin{array}{ll}7.2 & \text { Case I: Minkowski mode } \\ \end{array}$

$\begin{array}{lll}7.2 .1 & (1+1) \text {-dimensions } & 27\end{array}$

$\begin{array}{lll}7.2 .2 & (1+3) \text {-dimensions } & 30\end{array}$

7.3 Case II: Rindler mode $\quad 34$

7.3.1 (1+1)-dimensions 34

$\begin{array}{lll}\text { 7.3.2 (1+3)-dimensions } & 37\end{array}$

7.4 Summarizing the outcomes regarding the anti-Unruh(-like) effect 39

$\begin{array}{lll}8 & \text { Discussion } & 39\end{array}$

A Different Green's functions and the consequence of their consideration $\mathbf{4 2}$

A.1 Analogy between Green's functions of uniformly accelerated and static in thermal bath observers 
A.2 Analogy between Green's functions of accelerated atoms in thermal bath considering the Minkowski or Rindler modes, and atoms with double acceleration

B Green's function of accelerated observer considering Rindler modes
C Green's function of accelerated observer in thermal bath considering Rindler modes

D Relation between the detector proper times

\section{Introduction}

Quantum entanglement is one of the most distinguishing features that differentiates quantum physics from the classical. The fact that an entangled state of a collective system cannot be separated into the product states of the subsystems, acts as the essence of entanglement. It ensures that measurements of a physical observable on entangled particles are not independent of each other. The existence of quantum entanglement is experimentally verified in systems with photons, electrons, etc., see [1, 2]. Moreover, the application of quantum entanglement in quantum communications, cryptography, and computing $[3,4]$ has made it a more active and desirable field to venture further.

Furthermore, the realization and application of entanglement in flat and curved spacetimes through the usage of quantum field theory is considered to be the most enthralling recent outcomes, and there has been a growing interest in studying these relativistic quantum entanglement effects in recent times, see [5-16]. Another interesting phenomena is harvesting vacuum entanglement [6, 11, 17-19], i.e., quantum fields can be source for entanglement for atoms interacting with it. The degradation of entanglement due to uncontrolled coupling to the external field, resulting from the influence of the field-atom interaction, is also an actual physical problem in a realistic experimental situation. Then it becomes imperative to understand the reasons of these degradation, so that sincere predictions can be provided. All these reasonings motivated the developments on studying the transition rates between different states of entangled atoms in different trajectories, which are thriving with many new ideas and possibilities, see [20-35]. In this purpose the concept of two-level atomic Unruh-deWitt detectors are essential. These point-like atomic detectors, whose internal states are coupled to the external field, were conceptualized to understand the Unruh effect $[36,37]{ }^{1}$ To be specific, the Unruh effect proclaims that the Minkowski vacuum as perceived by a uniformly accelerated observer will present a Planckian distribution of particles, with the temperature proportional to the acceleration

\footnotetext{
${ }^{1}$ The Unruh-deWitt detector setup has been used to investigate whether a freely falling observer can detect particles in Boulware vacuum, both for black hole [38, 39] as well as Friedmann-Lamaître-RobertsonWalker [39] spacetimes. Moreover, Unruh effect has also been used to verify the quantum memory of the spacetime $[40,41]$.
} 
of the observer. There are plenty of works predicting the possibility of generating entangled states in systems of these two-level atoms interacting with bosonic and other fields [42-44], further enriching the plausibility of these experiments. It is to be noted that there are articles discussing the transition probabilities of entangled atoms in different scenarios for the static or accelerated observers. Like, in [24] the radiative process of the static atoms are discussed in the presence of mirrors. While in [20] the finite time effects of acceleration are analyzed. These studies motivates one to study the similar radiative process for entangled atoms accelerated in thermal bath, which is not there in literature up to our knowledge.

In this work we are going to study the radiative process of two entangled accelerated atoms, coupled to a background scalar field, in a thermal bath. The transition probability corresponding to single accelerated observers in thermal bath are estimated in $[45,46]$, considering two-level Unruh-deWitt detectors (where in [47] the scenario for a rotating detector in thermal bath is considered). In the derivation of [46] the Green's function, which is essential for the calculation, is constructed from the Minkowski modes with a Rindler coordinate transformation. These Green's functions are not time translation invariant and one cannot provide the notion of transition probability per unit time out of them. However, one can get the idea about the transition probability for certain field mode frequency from these estimations. We have considered this particular procedure to study the transition probability of entangled atoms accelerated in thermal bath in $(1+1)$ and $(1+3)$ dimensions.

On the other hand, we have also constructed the Green's functions out of the Rindler modes with the Unruh operators, i.e., with vacuum for Unruh modes(which is the Minkowski vacuum here), corresponding to accelerated observers in thermal bath, in both $(1+1)$ and $(1+3)$ dimensions. These Green's functions are time translational invariant and one can get the notion of transition probability per unit time out of them. In both of the cases with the Minkowski and Rindler modes we considered studying the transition probabilities for the transitions form the symmetric and anti-symmetric Bell states to the collective excited or ground state. We observed that these transition probabilities decreases with increasing detector acceleration in certain cases, the so called anti-Unruh(like) effect $^{2}[48,49]$ (this will be discussed later elaborately). We have further provided a thorough study about this effect in this work and tried to understand the source of this effect in our case. For the case with the Minkowski modes we inferred that the presence of the thermal bath, not the entanglement between the detectors, has a significant contribution in the occurrence of this anti-Unruh-like effect. On the other hand, for the case with the Rindler modes, where the anti-Unruh effect arises only for the transition from the anti-symmetric Bell state to the collective excited or ground state, the investigations suggest that the entanglement has the major role in the occurrence of the anti-Unruh phenomenon. Interestingly, while for the Minkowski mode case features of the results in $(1+1)$

\footnotetext{
${ }^{2}$ We mention that one cannot provide the notion of transition probability per unit time here for the case with the Minkowski modes as the Wightman function is not time translational invariant. On the other hand, the Unruh or anti-Unruh effect notion is always associated with a transition rate. Therefore, for Minkowski modes, we term them as Unruh-like and anti-Unruh-like effects to make a distinction from the usual notion. Whereas, for Rindler modes, as we will see later, one can provide transition rate expressions. Therefore, for this later discussion we reserve the phrases Unruh and anti-Unruh.
} 
and $(1+3)$ dimensions are not completely identical with each other, in the Rindler mode case the features are common in both dimensions. The consideration of these particular Rindler modes then provides further insights into the picture.

On the other hand, it is well known that the Minkowski vacuum fluctuations as seen by an uniformly accelerated observer has striking resemblance with that of the thermal fluctuations of a static observer in thermal bath. In this regard, there are many works in the direction of understanding the distinguishability and indistinguishability between them, see [46, 50-55]. In [52] the analogy between the two was pointed out by showing the satisfaction of the fluctuation dissipation theorem by the force due to radiation as measured by the accelerated frame (see [53] for this analogy in de-Sitter, FriedmannLamaître-Robertson-Walker background and [56] for the consideration of anomalous stress tensor). Furthermore, in $[46,50,51]$ the indistinguishability is proclaimed in certain scenarios. In particular, two different observers one accelerated in thermal bath and another with double acceleration, i.e., in the Rindler-Rindler frame, are shown to be analogous by studying the spectrum of observed particles. Here Bogoliubov transformation is utilized to obtain the spectrum of particles seen by a Rindler-Rindler observer in the Minkowski vacuum state and the detector response is studied to get spectrum of particles for an observer accelerated in a thermal bath. In [54] some significant dintinguishabilities between the observer static in thermal bath and the one with uniform acceleration are provided by studying different components of the renormalized stress energy tensor, and comparing them for Rindler-Rindler to Thermal-Rindler cases. All these analysis suggest the absence of a straight resolution out of this issue. We expected our analysis to shed some light on this matter too. In particular, we observed that in the calculation of obtaining the transition probabilities corresponding to the two-atom system there are also quantities that resemble the situation signifying the case of a single two-level detector accelerated in a thermal bath interacting with a scalar field. Namely we shall be denoting these quantities by the transition coefficients $\mathcal{F}_{11}$ for the Minkowski mode case and $R_{11}$ for the Rindler mode case. We observed that the quantity $\mathcal{F}_{11}$ is not symmetric under the interchange between the temperature of the thermal bath and the Unruh temperature. However, for the case with the Rindler modes with the vacuum of Unruh modes $R_{11}$ is symmetric under the same interchange. Thus suggesting this particular case may be the ideal representation for an accelerated observer, where the analogy with a thermal bath is prominent.

In section 2 we begin with a brief discussion of our model set-up, the two entangled two-level atoms coupled with the vacuum scalar field. In this section by perturbatively expanding the time evolution operator up to first order in the coupling constant the expression of the transition amplitude is provided. From the expression of these transition amplitudes and subsequently from the transition probabilities the role of the Green's functions corresponding to the detectors will be evident. Next, in section 3 the expressions of the Green's functions corresponding to uniformly accelerated observers in thermal bath are given considering the Minkowski modes. In section 4 the expressions of Green's functions for the same systems, considering the Rindler modes (with Unruh creation and annihilation operators), are given. In section 5 we have estimated the transition probabilities for the transitions from the entangled states to the collective excited state in the two-atom 
system considering the Green's functions of section 3. Subsequently, in section 6 we have considered the Green's functions from section 4 and estimated the transition probabilities for the same transitions. In section 7 we have studied the anti-Unruh(-like) effect resulting in the transition probabilities from the both cases considering the Minkowski and Rindler modes. We have concluded this article with a discussion of our results in section 8 .

\section{Radiative process of two entangled atoms: a model set-up}

We begin our analysis elucidating on the radiative process of two entangled Unruh-DeWitt detectors. This model has been taken up earlier in several situations [20, 24, 25]. Since we need this, a brief review of it will be presented here in order to make the discussion self-sufficient. Also this will help us to have a clear picture of the notations which we will introduce in order to define different quantities in the analysis.

The detectors are composed of point like two-level atoms, which are interacting with a massless, minimally coupled scalar field $\Phi(X)$ through monopole interaction. The Hamiltonian of this system of two two-level detectors interacting with the scalar field is expressed as

$$
H=H_{A}+H_{F}+H_{\text {int }}
$$

where, $H_{A}$ denotes the atomic Hamiltonian free of any interaction, $H_{F}$ is the free scalar field Hamiltonian, and $H_{\text {int }}$ is the interaction between the atoms and the scalar field. As provided by Dicke $[20,57]$ one may express the two-atom Hamiltonian corresponding to the proper time as

$$
H_{A}=\omega_{0}\left[S_{1}^{z} \otimes \mathbb{1}_{2} \frac{d \tau_{1}}{d \tau}+\mathbb{1}_{1} \otimes S_{2}^{z} \frac{d \tau_{2}}{d \tau}\right]
$$

where, $S_{j}^{z}=(1 / 2)\left(\left|e_{j}\right\rangle\left\langle e_{j}|-| g_{j}\right\rangle\left\langle g_{j}\right|\right)$ denotes the energy operator, with $\left|g_{j}\right\rangle$ and $\left|e_{j}\right\rangle$ respectively representing the ground and excited states of the $j^{\text {th }}$ atom with $j=1,2$ here. In eq. (2.2), $\mathbb{1}_{j}$ denote identity matrices, and $\omega_{0}$ the transition frequency corresponding to the collective two atom system. In particular for two identical atomic detectors the two-atom system has energy eigenvalues and the corresponding eigenstates, see [24], as

$$
\begin{aligned}
E_{e} & =\omega_{0}, & |e\rangle & =\left|e_{1}\right\rangle\left|e_{2}\right\rangle ; \\
E_{s} & =0, & |s\rangle & =\frac{1}{\sqrt{2}}\left(\left|e_{1}\right\rangle\left|g_{2}\right\rangle+\left|g_{1}\right\rangle\left|e_{2}\right\rangle\right) ; \\
E_{a} & =0, & |a\rangle & =\frac{1}{\sqrt{2}}\left(\left|e_{1}\right\rangle\left|g_{2}\right\rangle-\left|g_{1}\right\rangle\left|e_{2}\right\rangle\right) ; \\
E_{g} & =-\omega_{0}, & |g\rangle & =\left|g_{1}\right\rangle\left|g_{2}\right\rangle ;
\end{aligned}
$$

where, $|g\rangle$ and $|e\rangle$ respectively correspond to the ground and excited states of the collective system and $|s\rangle,|a\rangle$ denote the symmetric and anti-symmetric maximally entangled Bell states. A pictorial representation of them is shown in figure 1.

In (2.2), $\tau_{1}$ and $\tau_{2}$ are proper times corresponding to the frames attached with the first and second atomic detectors, respectively while $\tau$ denotes that for our observer who will measure the transition amplitudes. 


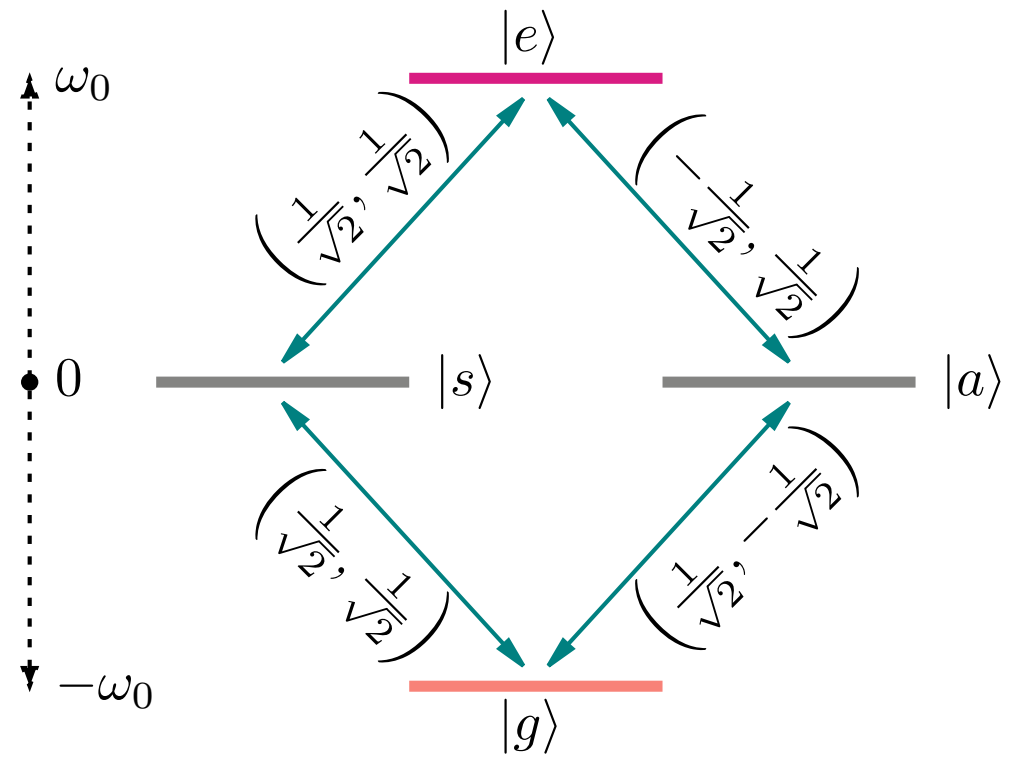

Figure 1. The energy levels corresponding to the eigenstates of the collective two-level two-atom system is depicted in this diagram. This figure has been taken from [25]. The contributions from the monopole moment for each transition are also noted.

In Minkowski spacetime the free Hamiltonian of the massless scalar field is

$$
H_{F}=\frac{1}{2} \int d^{3} X\left[(\dot{\Phi}(X))^{2}+|\nabla \Phi(X)|^{2}\right],
$$

where, the overhead dot denotes differentiation with respect to the time coordinate, and $\nabla$ denotes the vector differential operator. The monopole interaction Hamiltonian is given by

$$
H_{\mathrm{int}}(\tau)=\sum_{j=1}^{2} \mu_{j} \kappa_{j}\left(\tau_{j}(\tau)\right) m^{j}\left(\tau_{j}(\tau)\right) \Phi\left(X_{j}\left(\tau_{j}(\tau)\right)\right) \frac{d \tau_{j}(\tau)}{d \tau},
$$

where, $\mu_{j}$ denote the individual coupling between the detectors and the scalar field. On the other hand, $\kappa_{j}\left(\tau_{j}(\tau)\right)$ and $m^{j}\left(\tau_{j}(\tau)\right)$ respectively denote the switching function, and the detector monopole operators. For identical atomic detectors the coupling constants between different detectors and the scalar field can be assumed to be the same, i.e., $\mu_{1}=\mu_{2}=\mu$. With this consideration the time evolution operator can be expressed as

$$
\begin{aligned}
U=\mathcal{T} \exp \left\{-i \int_{-\infty}^{\infty} d \tau \mu\right. & {\left[\kappa_{1}\left(\tau_{1}(\tau)\right) m^{1}\left(\tau_{1}(\tau)\right) \Phi\left(X_{1}\left(\tau_{1}(\tau)\right)\right) \frac{d \tau_{1}(\tau)}{d \tau}\right.} \\
+ & \left.\left.\kappa_{2}\left(\tau_{2}(\tau)\right) m^{2}\left(\tau_{2}(\tau)\right) \Phi\left(X_{2}\left(\tau_{2}(\tau)\right)\right) \frac{d \tau_{2}(\tau)}{d \tau}\right]\right\}
\end{aligned}
$$

where $\mathcal{T}$ signifies that time ordering is done.

We consider $|\omega\rangle$ to be some collective initial state of the two detector system, and $|\Omega\rangle$ some collective final state. It is also considered that the initial state $|\omega\rangle$ is prepared in the Minkowski vacuum state $\left|0_{M}\right\rangle$ of the scalar field, and the final state $|\Omega\rangle$ evolves to some 
field state $|\Theta\rangle$. Then the transition amplitude from the state $\left|\omega ; 0_{M}\right\rangle$ to $|\Omega ; \Theta\rangle$, at the first order perturbation level of the coupling constant $\mu$, will be

$$
\begin{aligned}
\mathcal{A}_{\left|\omega ; 0_{M}\right\rangle \rightarrow|\Omega ; \Theta\rangle} & =\left\langle\Omega ; \Theta|U| \omega ; 0_{M}\right\rangle \\
& \approx-i \mu\left\langle\Omega ; \Theta\left|\int_{-\infty}^{\infty} d \tau\left[\kappa_{1} m^{1} \Phi\left(X_{1}\right) \frac{d \tau_{1}}{d \tau}+\kappa_{2} m^{2} \Phi\left(X_{2}\right) \frac{d \tau_{2}}{d \tau}\right]\right| \omega ; 0_{M}\right\rangle .
\end{aligned}
$$

From this transition amplitude one can obtain the transition probability for all possible field states $\{|\Theta\rangle\}$ as

$$
\Gamma_{|\omega\rangle \rightarrow|\Omega\rangle}=\sum_{\{|\Theta\rangle\}} \mathcal{A}_{\left|\omega ; 0_{M}\right\rangle \rightarrow|\Omega ; \Theta\rangle}^{*} \mathcal{A}_{\left|\omega ; 0_{M}\right\rangle \rightarrow|\Omega ; \Theta\rangle} \approx \mu^{2} \sum_{j, l=1}^{2} m_{\Omega \omega}^{j *} m_{\Omega \omega}^{l} F_{j l}(\Delta E),
$$

where $\Delta E=E_{\Omega}-E_{\omega}$, and $m_{\Omega \omega}^{j}=\left\langle\Omega\left|m^{j}(0)\right| \omega\right\rangle$. The monopole moment operator is defined as

$$
m^{j}(0)=\left|e_{j}\right\rangle\left\langle g_{j}|+| g_{j}\right\rangle\left\langle e_{j}\right|,
$$

and one can utilize this expression to acquire the contributions due to individual transitions through the monopole moments in the expression of the transition probability. In particular one can find out $m_{s e}^{1}=m_{s e}^{2}=1 / \sqrt{2}, m_{a e}^{1}=-m_{a e}^{2}=-1 / \sqrt{2}$, which respectively denote the contributions from the monopole moments due to the transitions from the symmetric and anti-symmetric states to the collective excited state. On the other hand, the contributions due to the transitions from the symmetric and anti-symmetric states to the collective ground state are $m_{s g}^{1}=m_{s g}^{2}=1 / \sqrt{2}$, and $m_{a g}^{2}=-m_{a g}^{1}=-1 / \sqrt{2}$. It can be observed that the transition from the collective excited to the ground state or reverse is not possible as in that case $m_{e g}^{j}=0=m_{g e}^{j}$. One can look into figure 1 , taken from [25], for a diagramatic representation of the energy levels and for the monopole operator expectation values corresponding to different transitions.

Let us now shift our attention to the transition coefficients in eq. (2.8), the explicit form of which is given by

$$
F_{j l}(\Delta E)=\int_{-\infty}^{\infty} d \tau d \tau^{\prime} e^{-i\left(\tau_{j}(\tau)-\tau_{l}\left(\tau^{\prime}\right)\right) \Delta E} G_{j l}^{+}\left(\tau_{j}(\tau), \tau_{l}\left(\tau^{\prime}\right)\right) \frac{d \tau_{j}}{d \tau} \frac{d \tau_{l}}{d \tau^{\prime}} \kappa_{j} \kappa_{l},
$$

where, the positive frequency Wightman function is defined as

$$
G_{j l}^{+}\left(\tau_{j}(\tau), \tau_{l}\left(\tau^{\prime}\right)\right)=\left\langle 0_{M}\left|\Phi\left[X_{j}\left(\tau_{j}(\tau)\right)\right] \Phi\left[X_{l}\left(\tau_{l}\left(\tau^{\prime}\right)\right)\right]\right| 0_{M}\right\rangle .
$$

Later we shall evaluate these transition coefficients specifically considering the first detector to be the place where our observer is located, i.e., then $\tau$ shall become $\tau_{1}$. It will result in a factor of $d \tau_{2}\left(\tau_{1}\right) / d \tau_{1}$ when one considers the contribution of the second detector, see $[20,25]$. Now we shall proceed to evaluate the transition probabilities for the detectors, uniformly accelerating with different accelerations, in a thermal bath. For this the explicit expression for the Wightman function is required which we will discuss in the next couple of sections. Since the field mode functions with respect to uniformly accelerated frame can be represented by using Minkowski as well as Rindler decomposition, below both will be discussed. We also mention that in our subsequent analysis we are going to consider the switching function $\kappa_{j}=1$. 


\section{Thermal Wightman function corresponding to Minkowski mode}

In this section we first consider a quantum statistical system of finite temperature and evaluate the Wightman function corresponding to positive frequency mode of free massless real scalar field with respect to Minkowski coordinates. We call these modes as Minkowski modes and with respect to them the vacuum is the usual Minkowski vacuum. Then we discuss about the Rindler spacetime, which corresponds to a uniformly accelerated object. We complete the section with consideration of the accelerated detectors in a thermal bath and by constructing the required form of Wightman functions, necessary for the evaluation of the transition probability in these trajectories.

\subsection{An observer in a thermal bath}

We take an observer to be in equilibrium with a thermal bath characterized by the parameter $\beta=1 /\left(k_{B} T\right)$, with $k_{B}$ being the Boltzmann constant and $T$ the temperature of the thermal bath. In this background we further consider a massless minimally coupled scalar field $\Phi(X)=\Phi(T, \mathbf{X})$. Then one can obtain the thermal Green's (Wightman) function by taking Gibbs ensemble average of the operator $\Phi\left(X_{2}\right) \Phi\left(X_{1}\right)$ as

$$
G_{\beta}^{+}\left(X_{2} ; X_{1}\right)=\left\langle\Phi\left(X_{2}\right) \Phi\left(X_{1}\right)\right\rangle_{\beta}=\frac{1}{Z} \operatorname{Tr}\left[e^{-\beta H} \Phi\left(X_{2}\right) \Phi\left(X_{1}\right)\right],
$$

where, $X_{1}$ and $X_{2}$ are two events in the spacetime and $Z=\operatorname{Tr}[\exp (-\beta H)]$ denotes the partition function. Here $H$ is the Hamiltonian of free massless scalar field (earlier we denoted this as $H_{F}$ in (2.4)). In Minkowski spacetime, using the standard Fock quantization, the scalar field can be expressed in terms of the annihilation and creation operators $a_{n}, a_{n}^{\dagger}$ and the positive and negative frequency mode functions as

$$
\Phi(X)=\sum_{k} \frac{f_{k}(\mathbf{X})}{\sqrt{2 \omega_{k}}}\left(a_{k} e^{-i \omega_{k} T}+a_{k}^{\dagger} e^{i \omega_{k} T}\right) .
$$

In Fourier domain the scalar field Hamiltonian acts as a sum of infinitely many simple Harmonic oscillators. Then one can consider $H_{k}=a_{k}^{\dagger} a_{k} \omega_{k}$ as the Hamiltonian operator corresponding to the $k^{\text {th }}$ excitation, and express the partition function to be $Z=\operatorname{Tr}\left[e^{-\beta H}\right]=\left(1-e^{-\beta \omega_{k}}\right)^{-1}$. Furthermore, the thermal Wightman function $[54,58,59]$ from eq. (3.1) can be expressed as

$$
G_{\beta}^{+}\left(X_{2} ; X_{1}\right)=\sum_{k} \frac{f_{k}\left(\mathbf{X}_{2}\right) f_{k}^{*}\left(\mathbf{X}_{1}\right)}{2 \omega_{k}}\left[\frac{e^{i \omega_{k} \Delta T}}{e^{\beta \omega_{k}}-1}+\frac{e^{-i \omega_{k} \Delta T}}{1-e^{-\beta \omega_{k}}}\right],
$$

where, $\Delta T=T_{2}-T_{1}$, and $T_{2} \geq T_{1}$ is considered. From this expression the notion of positive frequency Green's function is implicit.

In a $(1+1)$ dimensional spacetime the mode functions are $f_{k}(\mathbf{X})=(1 / \sqrt{2 \pi}) e^{i k X}$, and taking the discrete to continuous momentum limit one can express the thermal Green's function as

$$
G_{\beta}^{+}\left(X_{2} ; X_{1}\right)=\int \frac{d k}{4 \pi \omega_{k}}\left[\frac{e^{i k \Delta X+i \omega_{k} \Delta T}}{e^{\beta \omega_{k}}-1}+\frac{e^{i k \Delta X-i \omega_{k} \Delta T}}{1-e^{-\beta \omega_{k}}}\right] .
$$


One can explicitly perform the momentum integration in the expression of this Green's function, see [54] (we give this in appendix A; see eq. (A.1)).

Similarly, in $(1+3)$ dimensions the mode functions are $f_{n}(\mathbf{X})=\left(1 / \sqrt{(2 \pi)^{3}}\right) e^{i \mathbf{k} \cdot \mathbf{X}}$, and taking the discrete to continuous momentum limit the thermal Green's function becomes

$$
G_{\beta}^{+}\left(X_{2} ; X_{1}\right)=\int \frac{d^{3} k}{(2 \pi)^{3} 2 \omega_{k}}\left[\frac{e^{i \mathbf{k} . \Delta \mathbf{X}+i \omega_{k} \Delta T}}{e^{\beta \omega_{k}}-1}+\frac{e^{i \mathbf{k} \cdot \Delta \mathbf{X}-i \omega_{k} \Delta T}}{1-e^{-\beta \omega_{k}}}\right] .
$$

Here also the momentum integration can be explicitly carried out to provide a position space representation of the $(1+3)$ dimensional thermal Green's function, see [54] (see eq. (A.2) of appendix A). Below we shall express this in the frame of an uniformly accelerated observer. Since the Wightman functions are scalar quantities, one needs to just use coordinate transformations in order to obtain the same in accelerated frame.

\subsection{An accelerated observer in a thermal bath}

The coordinates of a uniformly accelerated object are confined to specific regions in Minkowski spacetime. These specific regions constitute the Rindler wedges [60] in a Minkowski spacetime. Like Minkowski spacetime these Rindler wedges also make up for static globally hyperbolic spacetimes. The motion of a uniformly accelerated observer is studied considering these Rindler frames.

Let us first consider a $(1+1)$-dimensional Minkowski spacetime denoted by the coordinates $(T, X)$, with the line element

$$
d s^{2}=-d T^{2}+d X^{2}
$$

The transformation to the coordinates $(\eta, \xi)$ in the right Rindler wedge (RRW), i.e., the region $|T|<X$ in the Minkowski spacetime, is

$$
\begin{aligned}
T & =\frac{e^{a \xi}}{a} \sinh a \eta \\
X & =\frac{e^{a \xi}}{a} \cosh a \eta .
\end{aligned}
$$

In a similar manner one can also define a coordinate transformation suitable to the left Rindler wedge (LRW), confined in a region $|T|<-X$ of the Minkowski spacetime. The line-elements corresponding to both of the right and left Rindler wedges are expressed as

$$
d s^{2}=e^{2 a \xi}\left[-d \eta^{2}+d \xi^{2}\right]
$$

It should be noted that the generalization of this $(1+1)$ dimensional Rindler transformation (3.7) and $(3.8)$ to $(1+3)$ dimensions, when the observer is accelerating along $X$-direction, is quite simple. The Minkowski $Y$ and $Z$ coordinates remain the same for an observer accelerated in the $X$ direction.

In Rindler spacetime the proper time can be estimated to be $\tau=e^{a \xi} \eta$ and proper acceleration $b=a e^{-a \xi}$. One can express the coordinate transformation from eq. (3.8) in 
terms of the proper time and proper acceleration as

$$
\begin{aligned}
T & =\frac{1}{b} \sinh b \tau \\
X & =\frac{1}{b} \cosh b \tau .
\end{aligned}
$$

As one considers $\xi=0$, then $\eta$ and $a$ respectively denote the proper time and acceleration of an accelerated observer.

Now we are going to consider accelerated observers in thermal background. The accelerated observers will be described by the Rindler coordinates. We begin by looking into the Green's function in this case. It can be seen that one can just use the Rindler transformation from eq. (3.9) and put the expressions of $\Delta T, \Delta X$ in eq. (A.1) and eq. (A.2) to get the Green's function in $(1+1)$ and $(1+3)$ dimensions for accelerated observers in thermal background. However, up to our knowledge, these forms of Green's functions, as not time translation invariant in accelerated frame, are not suitable to compute the transition coefficients of eq. (2.10). To circumvent this issue the Green's functions (3.4) and (3.5), where the momentum integration are not yet carried out, are considered (see e.g. [46], where this trick has been used).

In a $(1+1)$-dimensional thermal background one can express the thermal two-point function (3.4) in a different form with the consideration $\omega_{k}=|k|$ for a massless scalar field, which will be convenient for our calculations, as

$$
\begin{aligned}
G_{\beta}^{+}\left(X_{j} ; X_{l}\right)=\int_{0}^{\infty} \frac{d \omega_{k}}{4 \pi \omega_{k}}[ & \frac{e^{i \omega_{k}\left(\Delta T_{j l}-\Delta X_{j l}\right)}+e^{i \omega_{k}\left(\Delta T_{j l}+\Delta X_{j l}\right)}}{e^{\beta \omega_{k}}-1} \\
& \left.+\frac{e^{-i \omega_{k}\left(\Delta T_{j l}-\Delta X_{j l}\right)}+e^{-i \omega_{k}\left(\Delta T_{j l}+\Delta X_{j l}\right)}}{1-e^{-\beta \omega_{k}}}\right] .
\end{aligned}
$$

From eq. (3.9) one can obtain

$$
\begin{aligned}
& \Delta T_{j l}-\Delta X_{j l}=-\frac{1}{b_{j}} e^{-b_{j} \tau_{j}}+\frac{1}{b_{l}} e^{-b_{l} \tau_{l}} \\
& \Delta T_{j l}+\Delta X_{j l}=\frac{1}{b_{j}} e^{b_{j} \tau_{j}}-\frac{1}{b_{l}} e^{b_{l} \tau_{l}},
\end{aligned}
$$

where, $\Delta T_{j l}=T_{j, 2}-T_{l, 1}$ and $\Delta X_{j l}=X_{j, 2}-X_{l, 1}$. Here $j, l$ are the notations corresponding to different detectors and the subscript 1,2 denote different spacetime points. On the other hand, one can also consider the $(1+3)$ dimensional thermal Green's function as given in eq. (3.5) and get

$$
G_{\beta}^{+}\left(X_{j} ; X_{l}\right)=\int_{0}^{\pi} \sin \theta d \theta \int_{0}^{\infty} \frac{\omega_{k} d \omega_{k}}{2(2 \pi)^{2}}\left[\frac{e^{i \omega_{k}\left(\Delta X_{j l} \cos \theta+\Delta T_{j l}\right)}}{e^{\beta \omega_{k}}-1}+\frac{e^{i \omega_{k}\left(\Delta X_{j l} \cos \theta-\Delta T_{j l}\right)}}{1-e^{-\beta \omega_{k}}}\right],
$$

where, $\Delta T_{j l}$ and $\Delta X_{j l}$ are given by the previously mentioned expressions, and we have taken $\Delta Y_{j l}=0=\Delta Z_{j l}$ as the detectors are considered to be moving on the same $X-T$ plane. In particular, using the coordinate transformation from eq. (3.9) one can obtain

$$
\begin{aligned}
& X_{j} \cos \theta+T_{j}=\frac{1}{2 b_{j}}\left(\delta_{1} e^{b_{j} \tau_{j}}-\delta_{2} e^{-b_{j} \tau_{j}}\right) \\
& X_{j} \cos \theta-T_{j}=\frac{1}{2 b_{j}}\left(-\delta_{2} e^{b_{j} \tau_{j}}+\delta_{1} e^{-b_{j} \tau_{j}}\right),
\end{aligned}
$$


where, $\delta_{1}=1+\cos \theta$ and $\delta_{2}=1-\cos \theta$. One can put these expressions in eq. (3.12) to get the Green's function corresponding to accelerated observers in $(1+3)$ dimensional thermal bath. These expressions (eq. (3.10) and (3.12)) will be used for our later purpose. It should be mentioned that the $(1+1)$ dimensional Green's function of eq. (3.10) after the transformations (3.11) and the $(1+3)$ dimensional Green's function of eq. (3.12) with the transformations (3.13) do not remain time translation invariant. In the next section we are going to discuss about the Green's function for accelerating observers in thermal bath constructed considering the Rindler modes.

\section{Thermal Wightman function corresponding to Rindler modes}

The field can also be decomposed with respect to modes, defined in Rindler coordinates. The Rindler vacuum is the vacuum for these Rindler modes. Below we will find the thermal positive frequency Wightman function corresponding these modes in vacuum of the Unruh modes which is here Minkowski vacuum.

\section{$4.1(1+1)$ dimensions}

Let us start our discussion in $(1+1)$ dimensions. In terms of the Rindler coordinates the equation of motion for a minimally coupled, massless free scalar field $\Phi$ is $\square \Phi=$ $e^{-2 a \xi}\left(-\partial_{\eta}^{2} \Phi+\partial_{\xi}^{2} \Phi\right)=0$. The solution of this equation suggests a set of modes each in the right and left Rindler wedge as [61, 62]

$$
\begin{aligned}
{ }^{R} u_{k} & =\frac{1}{\sqrt{4 \pi \omega}} e^{i k \xi-i \omega \eta} & & \text { in RRW } \\
& =0 & & \text { in LRW } \\
{ }^{L} u_{k} & =\frac{1}{\sqrt{4 \pi \omega}} e^{i k \xi+i \omega \eta} & & \text { in LRW } \\
& =0 & & \text { in RRW. }
\end{aligned}
$$

In terms of these Rindler modes the scalar field can be expressed, see [61], as

$$
\Phi(X)=\sum_{k=-\infty}^{\infty}\left[b_{k}^{R} R u_{k}+b_{k}^{R^{\dagger}}{ }^{R} u_{k}^{*}+b_{k}^{L}{ }^{L} u_{k}+b_{k}^{L^{\dagger}}{ }^{L} u_{k}^{*}\right]
$$

where, superscript $L$ and $R$ denote modes and the operators corresponding to the left and the right Rindler wedges respectively. The operators correspond to Rindler vacuum $\left|0_{R}\right\rangle$, i.e. $b_{k}^{R}\left|0_{R}\right\rangle=0=b_{k}^{L}\left|0_{R}\right\rangle$. In particular, in the right Rindler wedge where the field modes ${ }^{L} u_{k}=0$ the scalar field takes the form

$$
\Phi^{R}(X)=\sum_{k=-\infty}^{\infty}\left[b_{k}^{R} R u_{k}+b_{k}^{R^{\dagger}} R u_{k}^{*}\right]
$$

We shall use this scalar field decomposition to obtain the Green's function with respect to the Minkowski vacuum for an accelerated observer described in the RRW. However, it should be noted that the operators $b_{k}^{R}$ and $b_{k}^{R^{\dagger}}$ in eq. (4.3) correspond to the Rindler vacuum $\left|0_{R}\right\rangle$. To circumvent this issue and to obtain the desired Green's function we seek 
help of a prescription provided by Unruh [36]. In the following discussions we are going to delineate this prescription namely the Unruh modes and the operators.

As defined in eq. (4.1) the field modes are separately non-vanishing in the two different Rindler wedges of the Minkowski spacetime. Unruh in 1976 provided a prescription out of these different modes which are valid in the whole region of the Minkowski spacetime. They are obtained from the combination of the Rindler modes ${ }^{R} u_{k}+e^{-\pi \omega / a} L_{u_{-k}^{*}}$ and $R_{u_{-k}^{*}}+e^{\pi \omega / a} L_{u_{k}}$. In terms of these modes the scalar field can be expressed as [61]

$$
\begin{aligned}
\Phi(X)=\sum_{k=-\infty}^{\infty} \frac{1}{\sqrt{2 \sinh \frac{\pi \omega}{a}}}[ & d_{k}^{1}\left(e^{\frac{\pi \omega}{2 a} R} u_{k}+e^{-\frac{\pi \omega}{2 a} L} u_{-k}^{*}\right) \\
& \left.+d_{k}^{2}\left(e^{-\frac{\pi \omega}{2 a} R} u_{-k}^{*}+e^{\frac{\pi \omega}{2 a} L} u_{k}\right)\right]+ \text { h.c. . }
\end{aligned}
$$

Here h.c. stands for Hermitian conjugate. It is observed that the Unruh modes have the positive frequency analyticity property corresponding to the Minkowski time, same as the Minkowski modes. Then the annihilation operators from the two sets of Unruh operators $\left(d_{k}^{1}, d_{k}^{1^{\dagger}}\right)$ and $\left(d_{k}^{2}, d_{k}^{2^{\dagger}}\right)$ annihilate the Minkowski vacuum

$$
d_{k}^{1}\left|0_{M}\right\rangle=d_{k}^{2}\left|0_{M}\right\rangle=0 .
$$

Because of this particular feature of eq. (4.5) it is now quite less cumbersome to obtain any Minkowski state expectation value. This can be achieved by transforming Rindler operators in terms of these Unruh operators, see [61], using the relations

$$
\begin{aligned}
& b_{k}^{L}=\frac{1}{\sqrt{2 \sinh \frac{\pi \omega}{a}}}\left[e^{\frac{\pi \omega}{2 a}} d_{k}^{2}+e^{-\frac{\pi \omega}{2 a}} d_{-k}^{1^{\dagger}}\right] \\
& b_{k}^{R}=\frac{1}{\sqrt{2 \sinh \frac{\pi \omega}{a}}}\left[e^{\frac{\pi \omega}{2 a}} d_{k}^{1}+e^{-\frac{\pi \omega}{2 a}} d_{-k}^{2^{\dagger}}\right],
\end{aligned}
$$

which is similar to the Bogoliubov transformation. Then putting this transformation in eq. (4.3) one can get the expression of the field in the RRW in terms of the Unruh operators as

$$
\Phi^{R}(X)=\sum_{k=-\infty}^{\infty} \frac{1}{\sqrt{2 \sinh \frac{\pi \omega}{a}}}\left[d_{k}^{1} e^{\frac{\pi \omega}{2 a} R} u_{k}+d_{k}^{2} e^{-\frac{\pi \omega}{2 a} R} u_{-k}^{*}\right]+h . c . .
$$

This expression of the scalar field in RRW is the same as the representation of eq. (4.4) with ${ }^{L} u_{k}$ taken to be zero.

First let us evaluate the Green's function for a single accelerated detector, without any thermal bath, considering the scalar field in RRW, i.e., the field decomposition from eq. (4.3) with Unruh operators. The field modes are expressed by eq. (4.7). Then one can obtain the Green's function, with $\omega=\omega_{k}=|k|$ considering the massless scalar field, for an accelerated observer with respect to the Rindler modes as

$$
G_{R}^{+}(\Delta \xi, \Delta \eta)=\int_{-\infty}^{\infty} \frac{d k}{4 \pi \omega_{k}}\left[\frac{e^{i k \Delta \xi-i \omega_{k} \Delta \eta}}{1-e^{\frac{-2 \pi \omega_{k}}{a}}}+\frac{e^{i k \Delta \xi+i \omega_{k} \Delta \eta}}{e^{\frac{2 \pi \omega_{k}}{a}}-1}\right] .
$$


In the above vacuum has been chosen to be that of Unruh mode, which is Minkowski vacuum here. For a derivation of this expression one can consider looking into appendix B. Comparing this expression (4.8) with that of the thermal Green's function (3.4), we notice that they are exactly the same with $\beta$ is now identified as $2 \pi / a$, and $\Delta \xi$ and $\Delta \eta$ resemble $\Delta T$ and $\Delta X$. Therefore, one can clearly proclaim that an accelerated observer with the Rindler modes in RRW mimics the thermal background.

On the other hand, one can obtain the Green's function corresponding to accelerated observers, with respect to Rindler modes, in a thermal bath in a similar manner. We consider the Hamiltonian corresponding to the $k^{\text {th }}$ excitation to be $H_{k}=\left(d_{k}^{1^{\dagger}} d_{k}^{1}+d_{k}^{2 \dagger} d_{k}^{2}\right) \omega_{k}$ (as the vacuum under study is that of Unruh mode) to evaluate the Green's function, defined by eq. (3.1) in a thermal background. This turns out to be

$$
\begin{aligned}
G_{\beta_{R}}^{+}\left(\Delta \xi_{j l}, \Delta \eta_{j l}\right)= & \int_{-\infty}^{\infty} \frac{d k}{8 \pi \omega_{k} \sqrt{\sinh \frac{\pi \omega_{k}}{a_{j}} \sinh \frac{\pi \omega_{k}}{a_{l}}}} \\
\times\left[\frac { 1 } { 1 - e ^ { - \beta \omega _ { k } } } \left\{e^{i k \Delta \xi_{j l}-i \omega_{k} \Delta \eta_{j l}} e^{\frac{\pi \omega_{k}}{2}\left(\frac{1}{a_{j}}+\frac{1}{a_{l}}\right)}\right.\right. & \left.+e^{i k \Delta \xi_{j l}+i \omega_{k} \Delta \eta_{j l}} e^{-\frac{\pi \omega_{k}}{2}\left(\frac{1}{a_{j}}+\frac{1}{a_{l}}\right)}\right\} \\
& \left.+\frac{1}{e^{\beta \omega_{k}}-1}\left\{e^{-i k \Delta \xi_{j l}+i \omega_{k} \Delta \eta_{j l}} e^{\frac{\pi \omega_{k}}{2}\left(\frac{1}{a_{j}}+\frac{1}{a_{l}}\right)}{ }^{-i k \Delta \xi_{j l}-i \omega_{k} \Delta \eta_{j l}} e^{-\frac{\pi \omega_{k}}{2}\left(\frac{1}{a_{j}}+\frac{1}{a_{l}}\right)}\right\}\right],
\end{aligned}
$$

where, $\Delta \xi_{j l}=\xi_{j, 2}-\xi_{l, 1}$ and $\Delta \eta_{j l}=\eta_{j, 2}-\eta_{l, 1}$. For a derivation of this expression one can look into appendix C. It should be noted that eq. (4.8) corresponds to the Green's function of a single accelerated observer expressed in terms of the Rindler modes. Whereas, eq. (4.9) corresponds to accelerated observers, generally considered to be of different accelerations, in thermal bath expressed in terms of the Rindler modes. In particular, when $a_{j}=a_{l}=a$ and $\beta \rightarrow \infty$ we get back the expression of eq. (4.8). Furthermore, one may consider $\xi_{j}=0$ then the proper accelerations of the observers are $b_{j}=a_{j}$ and the proper times $\tau_{j}=\eta_{j}$. Then one may also notice the clear difference of eq. (4.9) from its counter part eq. (3.10) after the transformation (3.11) corresponding to the Minkowski mode. The Green's function, coming from Rindler mode, is time translation invariant, whereas for the Minkowski modes it was not. We are going to use eq. (4.9) also to obtain the transition probabilities later.

\section{$4.2(1+3)$ dimensions}

In this subsection we talk about Rindler mode decomposition of the massless real scalar field in $(1+3)$ dimensions. The positive frequency mode solutions of the scalar field equation of motion $\square \Phi=0$ in $(1+3)$ dimensions in terms of the Rindler coordinates in the right 
and the left Rindler wedges are

$$
\begin{aligned}
R u_{\omega, k_{p}} & =\frac{1}{2 \pi^{2}} \sqrt{\frac{\sinh \left(\frac{\pi \omega}{a}\right)}{a}} \mathcal{K}\left[\frac{i \omega}{a}, \frac{\left|k_{p}\right| e^{a \xi}}{a}\right] e^{-i \omega \eta+i \overrightarrow{k_{p} \cdot \vec{x}}} & & \text { in RRW } \\
& =0 & & \text { in LRW } \\
{ }^{L} u_{\omega, k_{p}} & =\frac{1}{2 \pi^{2}} \sqrt{\frac{\sinh \left(\frac{\pi \omega}{a}\right)}{a}} \mathcal{K}\left[\frac{i \omega}{a}, \frac{\left|k_{p}\right| e^{a \xi}}{a}\right] e^{i \omega \eta+i \overrightarrow{k_{p} . \vec{x}}} & & \text { in LRW } \\
& =0 & & \text { in RRW },
\end{aligned}
$$

where, $\mathcal{K}[n, z]$ denotes the modified Bessel function of the second kind of order $n$, and $\vec{x}$ is perpendicular to the direction of acceleration, i.e., in the $Y-Z$ plane, see $[41,60,63]$. Here $\overrightarrow{k_{p}}$ denotes the transverse wave vector in the $Y-Z$ plane. Like the $(1+1)$ dimensional case here also one can decompose the scalar field confined to the right Rindler wedge as

$$
\Phi^{R}(X)=\sum_{\omega=0}^{\infty} \sum_{k_{p}=-\infty}^{\infty}\left[b_{\omega, k_{p}}^{R} R_{u_{\omega, k_{p}}}+b_{\omega, k_{p}}^{R^{\dagger}} R_{u_{\omega, k_{p}}}^{*}\right]
$$

where, the operators correspond to Rindler vacuum $\left|0_{R}\right\rangle$, i.e. $b_{\omega, k_{p}}^{R}\left|0_{R}\right\rangle=0$. Here also our main aim is to obtain the Green's function using the field decomposition of eq. (4.11) with respect to the Minkowski vacuum, which corresponds to an accelerating observer. In this regard, similar to the $(1+1)$ dimensional case one can utilize the concepts of the Unruh modes and operators. In particular, one can obtain the modes ${ }^{R} u_{\omega, k_{p}}+e^{-\pi \omega / a} L_{u_{\omega,-k_{p}}^{*}}$ and ${ }^{R} u_{\omega,-k_{p}}^{*}+e^{\pi \omega / a} L_{u_{\omega, k_{p}}}$ in terms of the Rindler modes which are valid in the whole Minkowski spacetime and have the positive frequency analyticity property with respect to the Minkowski time. In terms of these modes the scalar field is

$$
\begin{aligned}
\Phi(X)=\sum_{\omega=0}^{\infty} \sum_{k_{p}=-\infty}^{\infty} \frac{1}{\sqrt{2 \sinh \frac{\pi \omega}{a}}}[ & d_{\omega, k_{p}}^{1}\left(e^{\frac{\pi \omega}{2 a} R} u_{\omega, k_{p}}+e^{-\frac{\pi \omega}{2 a} L} u_{\omega,-k_{p}}^{*}\right) \\
& \left.+d_{\omega, k_{p}}^{2}\left(e^{-\frac{\pi \omega}{2 a} R} u_{\omega,-k_{p}}^{*}+e^{\frac{\pi \omega}{2 a} L} u_{\omega, k_{p}}\right)\right]+ \text { h.c. . }
\end{aligned}
$$

Here also the lowering operators from the two sets of Unruh annihilation and creation operators $\left(d_{\omega, k_{p}}^{1}, d_{\omega, k_{p}}^{1^{\dagger}}\right)$ and $\left(d_{\omega, k_{p}}^{2}, d_{\omega, k_{p}}^{2^{\dagger}}\right)$, annihilate the Minkowski vacuum

$$
d_{\omega, k_{p}}^{1}\left|0_{M}\right\rangle=d_{\omega, k_{p}}^{2}\left|0_{M}\right\rangle=0 .
$$

Then it will be helpful to express the scalar field in the RRW from eq. (4.11), using a relation analogous to eq. (4.6), in form

$$
\Phi^{R}(X)=\sum_{\omega=0}^{\infty} \sum_{k_{p}=-\infty}^{\infty} \frac{1}{\sqrt{2 \sinh \frac{\pi \omega}{a}}}\left[d_{\omega, k_{p}}^{1} e^{\frac{\pi \omega}{2 a} R} u_{\omega, k_{p}}+d_{\omega, k_{p}}^{2} e^{-\frac{\pi \omega}{2 a} R} u_{\omega,-k_{p}}^{*}\right]+\text { h.c. },
$$

in terms of the Unruh operators. It should be mentioned that like the $(1+1)$ dimensional case here also this field decomposition is exactly same as the Unruh field decomposition from eq. (4.12) with the Rindler mode corresponding to the left Rindler wedge ${ }^{L} u_{\omega, k_{p}}$ considered to be zero. Like before we evaluate the Green's function for accelerated detectors 
considering the field decomposition of eq. (4.14), where the accelerating observer is considered to be in the right Rindler wedge. Then the Green's function for an accelerated observer without thermal bath with respect to the Rindler modes is obtained as

$$
G_{R}^{+}(\Delta \xi, \Delta \eta)=\int_{0}^{\infty} \frac{\omega d \omega}{(2 \pi)^{2}}\left[\frac{e^{-i \omega \Delta \eta}}{1-e^{\frac{-2 \pi \omega}{a}}}+\frac{e^{i \omega \Delta \eta}}{e^{\frac{2 \pi \omega}{a}}-1}\right] e^{-2 a \xi_{0}}
$$

In deriving this expression in $(1+3)$ dimensions we have considered that the accelerated detector to be positioned at a fixed Rindler $\xi$ coordinate, $\xi=\xi_{0}$.

In a similar manner, one can obtain the Green's function corresponding to accelerated observers, with respect to Rindler modes in a thermal bath in $(1+3)$ dimensions. We use the RRW field decomposition from eq. (4.14) and take the Hamiltonian to be $H_{\omega, k_{p}}=$ $\left(d_{\omega, k_{p}}^{1^{\dagger}} d_{\omega, k_{p}}^{1}+d_{\omega, k_{p}}^{2^{\dagger}} d_{\omega, k_{p}}^{2}\right) \omega$ to evaluate the Green's function, defined by eq. (3.1) in a thermal background. This Green's function is

$$
\begin{aligned}
& G_{\beta_{R}}^{+}\left(\Delta \eta_{j l}\right)=\int_{0}^{\infty} d \omega \int \frac{d^{2} k_{p}}{(2 \pi)^{4}} \frac{2}{\sqrt{a_{j} a_{l}}} \\
& \times\left[\frac{e^{-i \omega \Delta \eta_{j l}} e^{\frac{\pi \omega}{2}\left(\frac{1}{a_{j}}+\frac{1}{a_{l}}\right)}+e^{i \omega \Delta \eta_{j l}} e^{-\frac{\pi \omega}{2}\left(\frac{1}{a_{j}}+\frac{1}{a_{l}}\right)}}{1-e^{-\beta \omega}}\right. \\
& \left.+\frac{e^{i \omega \Delta \eta_{j l}} e^{\frac{\pi \omega}{2}\left(\frac{1}{a_{j}}+\frac{1}{a_{l}}\right)}+e^{-i \omega \Delta \eta_{j l}} e^{-\frac{\pi \omega}{2}\left(\frac{1}{a_{j}}+\frac{1}{a_{l}}\right)}}{e^{\beta \omega}-1}\right] \\
& \times \mathcal{K}\left[\frac{i \omega}{a_{j}}, \frac{\left|k_{p}\right| e^{a_{j} \xi_{j}}}{a_{j}}\right] \mathcal{K}\left[\frac{i \omega}{a_{l}}, \frac{\left|k_{p}\right| e^{a_{l} \xi_{l}}}{a_{l}}\right],
\end{aligned}
$$

where, $\Delta \eta_{j l}=\eta_{j, 2}-\eta_{l, 1}$ and $\xi_{j}$ is the fixed Rindler spatial coordinate corresponding to the $j^{\text {th }}$ detector. Note again that the above one is time translational invariant. We are going to utilize these above mentioned considerations to obtain the transition probabilities of eq. (2.8) for two accelerated atoms immersed in a thermal bath considering the Rindler modes in $(1+3)$ dimensions.

\section{Transition probability for accelerated atoms in thermal bath with re- spect to Minkowski modes}

In this section we are going to estimate the transition probability from eq. (2.8) for two entangled atoms accelerated in a thermal bath. We shall consider here the $(1+1)$ and $(1+3)$ dimensional Green's functions (3.10) and (3.12) with the coordinate transformations (3.11) and (3.13), which correspond to accelerated detectors in thermal background with respect to the Minkowski modes. Our observer is considered to be co-moving with the first accelerated detector, and we perform all of our evaluations with respect to this first frame. The proper time of the first atom $\tau=\tau_{1}$ will be used to carry out the integration in eq. (2.10) to evaluate the transition coefficients. We consider for both of the atoms the 
Rindler parameter $a$ to be the same. Then for different atoms with different constant $\xi$ the proper accelerations $b_{1}$ and $b_{2}$ will be different with different proper times. This provides a spatial separation between them in the $X$-direction. Now if one considers both of the observers with respect to the same Rindler time $\eta$ one can obtain a relation between the proper times as

$$
\tau_{2}=\frac{b_{1}}{b_{2}} \tau_{1}
$$

See [20] and appendix D for details. We shall use this relation to evaluate the transition coefficients.

\section{$5.1(1+1)$ dimensions}

To evaluate the transition coefficients in $(1+1)$ dimensions for two entangled atoms accelerated in a thermal bath considering the Minkowski modes we consider the Green's function (3.10) and put it in eq. (2.10) with the coordinate transformation (3.11). Let us denote $\alpha_{j}=\left(b_{1} / b_{2}\right)^{\delta_{2, j}}$, where $\delta_{i, j}$ denotes the Kronecker delta with detector's indices $i$ and $j$. Then the proper time of the $j^{\text {th }}$ atom, by eq. (5.1), is given by $\tau_{j}=\alpha_{j} \tau_{1}$. One can then express the coefficient functions $F_{j l}(\Delta E)$ as

$$
\begin{aligned}
F_{j l}(\Delta E)=\int_{0}^{\infty} \frac{d \omega_{k}}{4 \pi \omega_{k}} \alpha_{j} \alpha_{l} & {\left[\frac{\mathcal{I}_{1}\left(b_{j}\right) \mathcal{I}_{1}^{*}\left(b_{l}\right)+\mathcal{I}_{2}\left(b_{j}\right) \mathcal{I}_{2}^{*}\left(b_{l}\right)}{e^{\beta \omega_{k}}-1}\right.} \\
+ & \left.\frac{\mathcal{I}_{3}\left(b_{j}\right) \mathcal{I}_{3}^{*}\left(b_{l}\right)+\mathcal{I}_{4}\left(b_{j}\right) \mathcal{I}_{4}^{*}\left(b_{l}\right)}{1-e^{-\beta \omega_{k}}}\right],
\end{aligned}
$$

where

$$
\begin{aligned}
& \mathcal{I}_{1}\left(b_{j}\right)=\int_{-\infty}^{\infty} d \tau_{1} e^{-i \Delta E \tau_{j}} \exp \left(-\frac{i \omega_{k}}{b_{j}} e^{-b_{j} \tau_{j}}\right) \\
& \mathcal{I}_{2}\left(b_{j}\right)=\int_{-\infty}^{\infty} d \tau_{1} e^{-i \Delta E \tau_{j}} \exp \left(\frac{i \omega_{k}}{b_{j}} e^{b_{j} \tau_{j}}\right) \\
& \mathcal{I}_{3}\left(b_{j}\right)=\int_{-\infty}^{\infty} d \tau_{1} e^{-i \Delta E \tau_{j}} \exp \left(\frac{i \omega_{k}}{b_{j}} e^{-b_{j} \tau_{j}}\right) \\
& \mathcal{I}_{4}\left(b_{j}\right)=\int_{-\infty}^{\infty} d \tau_{1} e^{-i \Delta E \tau_{j}} \exp \left(-\frac{i \omega_{k}}{b_{j}} e^{b_{j} \tau_{j}}\right) .
\end{aligned}
$$

To simplify these integral expressions one can make change of variables $e^{-b_{j} \tau_{j}}=y$ and $e^{b_{j} \tau_{j}}=z$, and we also use of the relation $\tau_{j}=\alpha_{j} \tau_{1}$ between the proper times corresponding to two differently accelerated observers. Then the integrals become

$$
\begin{aligned}
& \mathcal{I}_{1}\left(b_{j}\right)=\frac{1}{b_{j} \alpha_{j}} \int_{0}^{\infty} d z z^{-1+\frac{i \Delta E}{b_{j}}} e^{-\frac{i \omega_{k}}{b_{j}} z}=\frac{1}{b_{j} \alpha_{j}}\left(\frac{\omega_{k}}{b_{j}}\right)^{-\frac{i \Delta E}{b_{j}}} e^{\frac{\pi \Delta E}{2 b_{j}}} \Gamma\left(\frac{i \Delta E}{b_{j}}\right)=\mathcal{I}_{2}^{*}\left(b_{j}\right) \\
& \mathcal{I}_{3}\left(b_{j}\right)=\frac{1}{b_{j} \alpha_{j}} \int_{0}^{\infty} d z z^{-1+\frac{i \Delta E}{b_{j}}} e^{\frac{i \omega_{k}}{b_{j}} z}=\frac{1}{b_{j} \alpha_{j}}\left(\frac{\omega_{k}}{b_{j}}\right)^{-\frac{i \Delta E}{b_{j}}} e^{-\frac{\pi \Delta E}{2 b_{j}}} \Gamma\left(\frac{i \Delta E}{b_{j}}\right)=\mathcal{I}_{4}^{*}\left(b_{j}\right) .
\end{aligned}
$$

To perform the above integration we have used the formula

$$
\int_{0}^{\infty} d x x^{s-1} e^{-b x}=e^{-s \ln b} \Gamma(s),
$$


with the conditions $\operatorname{Re}(b)>0$ and $\operatorname{Re}(s)>0$. To ensure the convergence of our integrals the standard prescription has been adopted here (see e.g. [64] for details). Using these results one can express the coefficient functions in eq. (5.2) as $F_{j l}(\Delta E)=\int_{0}^{\infty} d \omega_{k} \mathcal{F}_{j l}\left(\Delta E, \omega_{k}\right)$. Here $\mathcal{F}_{j l}\left(\Delta E, \omega_{k}\right)$ denote the transition coefficients corresponding to each mode with wave number $k$. These transition coefficients are given by

$$
\mathcal{F}_{j l}\left(\Delta E, \omega_{k}\right)=\frac{\operatorname{Re}\left[\mathcal{C}_{1}\left(b_{j}, b_{l}\right)\right]}{2 \pi \omega_{k} b_{j} b_{l}}\left[\frac{e^{\frac{\pi \Delta E}{2}\left(\frac{1}{b_{j}}+\frac{1}{b_{l}}\right)}}{e^{\beta \omega_{k}}-1}+\frac{e^{-\frac{\pi \Delta E}{2}\left(\frac{1}{b_{j}}+\frac{1}{b_{l}}\right)}}{1-e^{-\beta \omega_{k}}}\right],
$$

where

$$
\mathcal{C}_{1}\left(b_{j}, b_{l}\right)=\left(\frac{\omega_{k}}{b_{j}}\right)^{-\frac{i \Delta E}{b_{j}}}\left(\frac{\omega_{k}}{b_{l}}\right)^{\frac{i \Delta E}{b_{l}}} \Gamma\left(\frac{i \Delta E}{b_{j}}\right) \Gamma\left(-\frac{i \Delta E}{b_{l}}\right) .
$$

From (5.6) and (5.7) one can obtain the expression of the transition coefficient $\mathcal{F}_{11}\left(\Delta E, \omega_{k}\right)$ as

$$
\mathcal{F}_{11}\left(\Delta E, \omega_{k}\right)=\frac{1}{\omega_{k} \Delta E b_{1}}\left[\frac{1}{e^{\beta \omega_{k}}-1} \frac{1}{1-e^{\frac{-2 \pi \Delta E}{b_{1}}}}+\frac{1}{1-e^{-\beta \omega_{k}}} \frac{1}{e^{\frac{2 \pi \Delta E}{b_{1}}}-1}\right],
$$

where, we have used the Gamma function identity $\Gamma(i z) \Gamma(-i z)=\pi /(z \sinh \pi z)$. Also eq. (5.6) and (5.7) shows that for equal acceleration $b_{2}=b_{1}$ all the transition coefficients $\mathcal{F}_{j l}\left(\Delta E, \omega_{k}\right)$ become equal to $\mathcal{F}_{11}\left(\Delta E, \omega_{k}\right)$. It should be mentioned that for a single detector, accelerated in thermal background, $\mathcal{F}_{11}\left(\Delta E, \omega_{k}\right)$ corresponds to the required transition probability. The same has been obtained earlier in [46]. Note that it is not symmetric under the exchange $\beta \leftrightarrow(2 \pi) / b_{1}$ due to the over all multiplicative factor $\left(1 / b_{1}\right)$. This originates in our calculation as it is based on the Minkowski mode. Later we will notice that Rindler modes do not give rise to this asymmetric property.

Now we shall evaluate the transition probability between different states of the twoatom system using eq. (2.8). For instance the transition probability from the symmetric entangled state $|s\rangle$ to the collective excited state $|e\rangle$ comes out to be $\Gamma_{s e}=\int_{0}^{\infty} d \omega_{k} \gamma_{s e}$, where the expression of $\gamma_{s e}$ is given by,

$$
\gamma_{s e}=\frac{\mu^{2}}{2}\left[\left\{\mathcal{F}_{11}\left(\omega_{0}, \omega_{k}\right)+\mathcal{F}_{22}\left(\omega_{0}, \omega_{k}\right)\right\}+\left\{\mathcal{F}_{12}\left(\omega_{0}, \omega_{k}\right)+\mathcal{F}_{21}\left(\omega_{0}\right), \omega_{k}\right\}\right]
$$

Whereas the same between the anti-symmetric state $|a\rangle$ to the excited state $|e\rangle$ is provided by

$$
\gamma_{a e}=\frac{\mu^{2}}{2}\left[\left\{\mathcal{F}_{11}\left(\omega_{0}, \omega_{k}\right)+\mathcal{F}_{22}\left(\omega_{0}, \omega_{k}\right)\right\}-\left\{\mathcal{F}_{12}\left(\omega_{0}, \omega_{k}\right)+\mathcal{F}_{21}\left(\omega_{0}, \omega_{k}\right)\right\}\right]
$$

It should be noted that in both of the above cases the change in energy level of the collective system is $\Delta E=\omega_{0}-0=\omega_{0}$. Furthermore, for the transitions from the symmetric and anti-symmetric states to the ground state one has $\Delta E=0-\left(-\omega_{0}\right)=\omega_{0}$. Then one can find out the transition probabilities from the symmetric and anti-symmetric states to the ground state, also provided by the $\gamma_{s e}$ and $\gamma_{a e}$, respectively. Also note that for equal proper acceleration of the two atoms the transition probability from the anti-symmetric state to 
the collective excited state or to the collective ground state becomes zero. The transition probability corresponding to the transition from the symmetric and anti-symmetric entangled states to the collective excited state as a function of $b_{1}$ are depicted in figure 2 .

It should be noted that in this figure we have plotted the quantities from eq. (5.9) and (5.10) per unit $\mu^{2} / 2$. Furthermore, in the subsequent studies also we shall take the same consideration. Here since the observer is attached with the first atom, we show the variation with respect to first atom's proper acceleration $b_{1}$. For the symmetric case, the entanglement between the states of atoms, acts as enhancement in the transition probability (the cross terms, e.g. $\mathcal{F}_{12}$ and $\mathcal{F}_{21}$ are added), whereas in the anti-symmetric situation entanglement provides decrease of transition probability. Interesting point to be noted from the figure that in both of the cases the transition coefficients at first tend to decrease with increasing acceleration $b_{1}$ of the first observer, giving rise to a possible case of the anti-Unruhlike effect, and then increases with it. Later, before providing our concluding remarks, we shall elaborately discuss about this phenomena of anti-Unruh-like effect in this scenario.

\section{$5.2(1+3)$ dimensions}

To estimate the transition coefficients between different states of a collective system of two entangled atoms accelerated in a thermal bath in $(1+3)$ dimensions one can put the expression of the Green's function (3.12) in eq. (2.10). With this and the substitution of coordinate transformations (3.13) one obtains

$$
F_{j l}(\Delta E)=\int_{0}^{\infty} d \omega_{k} \int_{0}^{\pi} \frac{\omega_{k} \sin \theta}{2(2 \pi)^{2}} d \theta \alpha_{j} \alpha_{l}\left[\frac{\mathcal{I}_{1_{3 D}}\left(b_{j}\right) \mathcal{I}_{1_{3 D}}^{*}\left(b_{l}\right)}{e^{\beta \omega_{k}}-1}+\frac{\mathcal{I}_{2_{3 D}}\left(b_{j}\right) \mathcal{I}_{2_{3 D}}^{*}\left(b_{l}\right)}{1-e^{-\beta \omega_{k}}}\right] .
$$

The integrals $\mathcal{I}_{1_{3 D}}\left(b_{j}\right)$ can be evaluated to be

$$
\mathcal{I}_{1_{3 D}}\left(b_{j}\right)=\int_{-\infty}^{\infty} d \tau_{1} e^{-i \tau_{j} \Delta E} e^{i \omega_{k}\left(X_{j} \cos \theta+T_{j}\right)}=\frac{2 e^{\frac{\pi \Delta E}{2 b_{j}}}}{b_{j} \alpha_{j}}\left(\frac{\delta_{1}}{\delta_{1}}\right)^{\frac{i \Delta E}{2 b_{j}}} \mathcal{K}\left[\frac{i \Delta E}{b_{j}}, \frac{\omega_{k} \sqrt{\delta_{1} \delta_{2}}}{b_{j}}\right],
$$

and integrals $\mathcal{I}_{2_{3 D}}\left(b_{j}\right)$ as

$$
\mathcal{I}_{1_{3 D}}\left(b_{j}\right)=\int_{-\infty}^{\infty} d \tau_{1} e^{-i \tau_{j} \Delta E} e^{i \omega_{k}\left(X_{j} \cos \theta-T_{j}\right)}=\frac{2 e^{-\frac{\pi \Delta E}{2 b_{j}}}}{b_{j} \alpha_{j}}\left(\frac{\delta_{1}}{\delta_{2}}\right)^{-\frac{i \Delta E}{2 b_{j}}} \mathcal{K}\left[\frac{i \Delta E}{b_{j}}, \frac{\omega_{k} \sqrt{\delta_{1} \delta_{2}}}{b_{j}}\right],
$$

where, $\delta_{1}=1+\cos \theta, \delta_{2}=1-\cos \theta$, and $\mathcal{K}[n, z]$ denotes the modified Bessel function of the second kind of order $n$. Then one express the coefficient functions like before as $F_{j l}(\Delta E)=\int_{0}^{\infty} d \omega_{k} \mathcal{F}_{j l}\left(\Delta E, \omega_{k}\right)$, where

$$
\begin{aligned}
\mathcal{F}_{j l}\left(\Delta E, \omega_{k}\right)=\int_{0}^{\pi} \sin \theta d \theta \frac{\omega_{k}}{2 \pi^{2} b_{j} b_{l}} \mathcal{C}_{2}\left(\theta, b_{j}, b_{l}\right) & {\left[\frac{e^{\frac{\pi}{2}\left(\frac{\Delta E}{b_{j}}+\frac{\Delta E}{b_{l}}\right)}}{e^{\beta \omega_{k}}-1}\left(\frac{\delta_{1}}{\delta_{2}}\right)^{\frac{i \Delta E}{2}\left(\frac{1}{b_{j}}-\frac{1}{b_{l}}\right)}\right.} \\
& \left.+\frac{e^{-\frac{\pi}{2}\left(\frac{\Delta E}{b_{j}}+\frac{\Delta E}{b_{l}}\right)}}{1-e^{-\beta \omega_{k}}}\left(\frac{\delta_{1}}{\delta_{2}}\right)^{-\frac{i \Delta E}{2}\left(\frac{1}{b_{j}}-\frac{1}{b_{l}}\right)}\right] .
\end{aligned}
$$




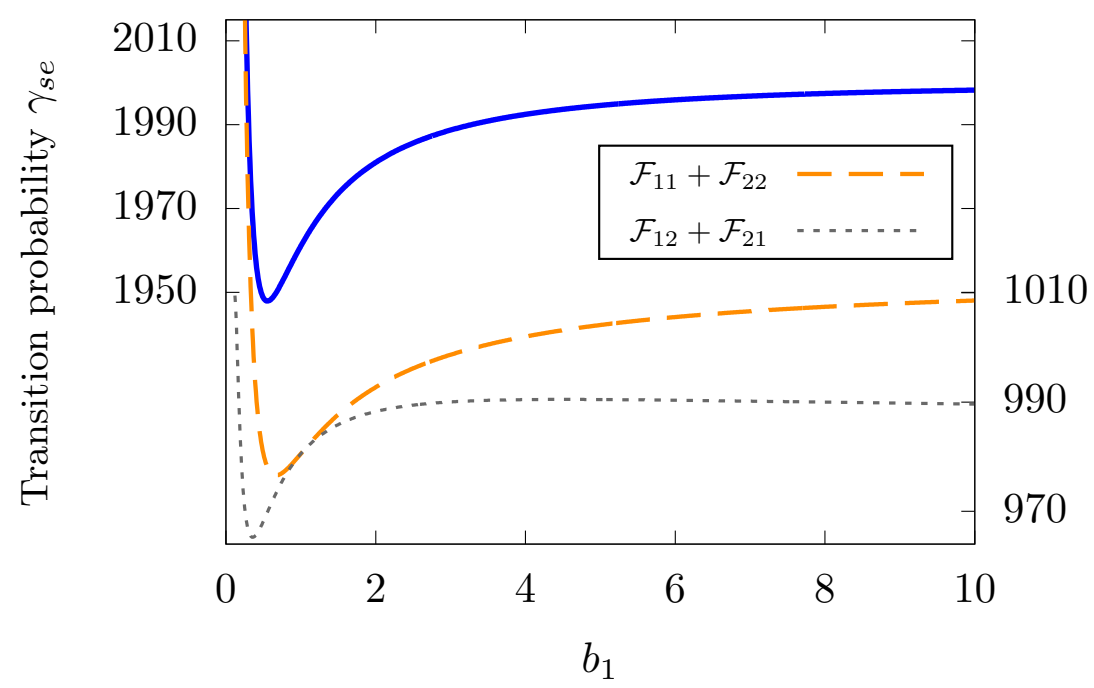

(a)

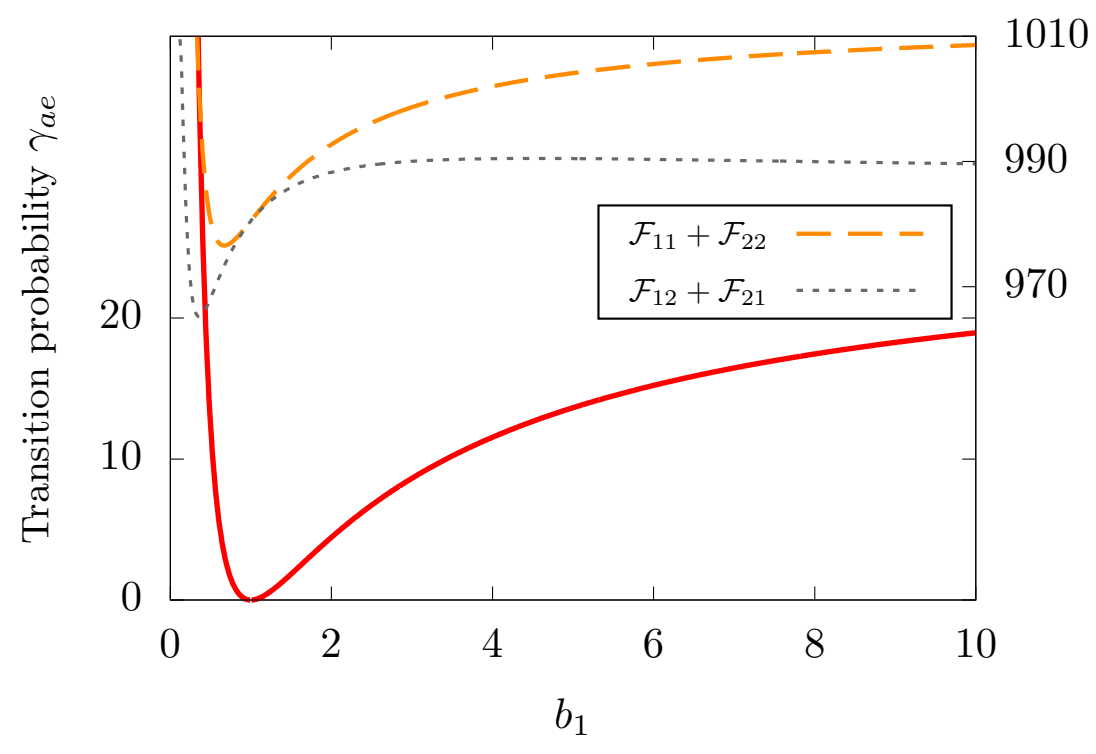

(b)

Figure 2. $(1+1)$ dimensions: (a) The transition probability from the symmetric state to the collective excited state denoted by the solid blue line. (b) The transition probability from the anti-symmetric state to the collective excited state denoted by the solid red line. In both of the plots the sum of the transition coefficients $\left(\mathcal{F}_{11}+\mathcal{F}_{22}\right)$ for the transitions from the symmetric or anti-symmetric states to the excited state are denoted by orange dashed lines. The sum of the transition coefficients $\left(\mathcal{F}_{12}+\mathcal{F}_{21}\right)$ for the transitions from the symmetric or anti-symmetric states to the excited state are denoted by gray dotted lines. In all of the above cases $b_{2}=1$ is kept fixed and $b_{1}$ is varied. The value of the other parameters are $\Delta E=0.1, \omega_{k}=0.1$ and $\beta=2 \pi$. 
and the quantity $\mathcal{C}_{2}\left(\theta, b_{j}, b_{l}\right)$ is given by

$$
\mathcal{C}_{2}\left(\theta, b_{j}, b_{l}\right)=\mathcal{K}\left[\frac{i \Delta E}{b_{j}}, \frac{\omega_{k} \sqrt{\delta_{1} \delta_{2}}}{b_{j}}\right]\left(\mathcal{K}\left[\frac{i \Delta E}{b_{l}}, \frac{\omega_{k} \sqrt{\delta_{1} \delta_{2}}}{b_{l}}\right]\right)^{*} .
$$

We found it to be suitable to perform this $\theta$ integration numerically and then to plot the resulting transition probabilities. However, for equal proper accelerations one can perform this $\theta$ integral analytically to express the transition coefficient $\mathcal{F}_{11}\left(\Delta E, \omega_{k}\right)$ as

$$
\mathcal{F}_{11}\left(\Delta E, \omega_{k}\right)=\frac{\omega_{k}}{4 \pi b_{1}^{2}}\left[\frac{1}{e^{\beta \omega_{k}}-1} \frac{1}{1-e^{\frac{-2 \pi \Delta E}{b_{1}}}}+\frac{1}{1-e^{-\beta \omega_{k}}} \frac{1}{e^{\frac{2 \pi \Delta E}{b_{1}}}-1}\right] \mathcal{C}_{3}\left(b_{1}\right),
$$

where the expression of $\mathcal{C}_{3}\left(b_{1}\right)$ is given by

$$
\begin{aligned}
\mathcal{C}_{3}\left(b_{1}\right)=\frac{b_{1}}{\Delta E_{2}} F_{3}\left(\frac{1}{2}, 1 ; \frac{3}{2}, 1\right. & \left.-\frac{i \Delta E}{b_{1}}, \frac{i \Delta E}{b_{1}}+1 ; \frac{\omega_{k}^{2}}{b_{1}^{2}}\right) \\
+2 \cosh \left(\frac{\pi \Delta E}{b_{1}}\right) \operatorname{Im} & {\left[\Gamma\left(-\frac{2 i \Delta E}{b_{1}}-1\right)\left(\frac{\omega_{k}}{b_{1}}\right)^{\frac{2 i \Delta E}{b_{1}}}\right.} \\
& \left.\times{ }_{1} F_{2}\left(\frac{i \Delta E}{b_{1}}+\frac{1}{2} ; \frac{i \Delta E}{b_{1}}+\frac{3}{2}, \frac{2 i \Delta E}{b_{1}}+1 ; \frac{\omega_{k}^{2}}{b_{1}^{2}}\right)\right] .
\end{aligned}
$$

Here ${ }_{p} F_{q}(m ; n ; z)$ denotes the generalized hypergeometric function. Note again that the above expression is not symmetric under the interchange $\beta \leftrightarrow(2 \pi) / b_{1}$ and as we mentioned earlier, it is due to our choice of mode which is Minkowski here.

One can get the transition probability for the transitions from the symmetric and antisymmetric states to the excited and ground states in this case as well. The expressions will be given by (5.9) and (5.10) again, but in this case $\mathcal{F}_{j l}$ are determined by (5.14). The Transition probabilities are depicted in figure 3 .

Note again that the entanglement provides enhancement in transition for the symmetric case whereas decrement to that for anti-symmetric situation. Here also the anti-Unruh-like effects are evident and we shall properly discuss about it later in this paper. But there is a distinct difference in the features of transition coefficients compared to $(1+1)$ dimensional case. Here $\gamma_{s e}$ shows anti-Unruh-like phenomenon for all values of $b_{1}$ while this is not true in $(1+1)$ dimensions in same parameter range. It should also be noted that all of the transition coefficients become equal to $\mathcal{F}_{11}\left(\Delta E, \omega_{k}\right)$ in the equal acceleration case $b_{2}=b_{1}$ and the transition probabilities from the anti-symmetric state to the collective excited or ground states become zero.

\section{Transition probability for accelerated atoms with the Rindler modes in a thermal background}

The scalar field in terms of the Rindler modes in RRW are meant to resemble the thermal characteristics with respect to Minkowski vacuum for an accelerated observer. From the $(1+1)$ and $(1+3)$ dimensional field decompositions (4.4) and (4.12), and the Green's functions (4.8), (4.9) and (4.15), (4.16) it can be observed that they are all represented in 


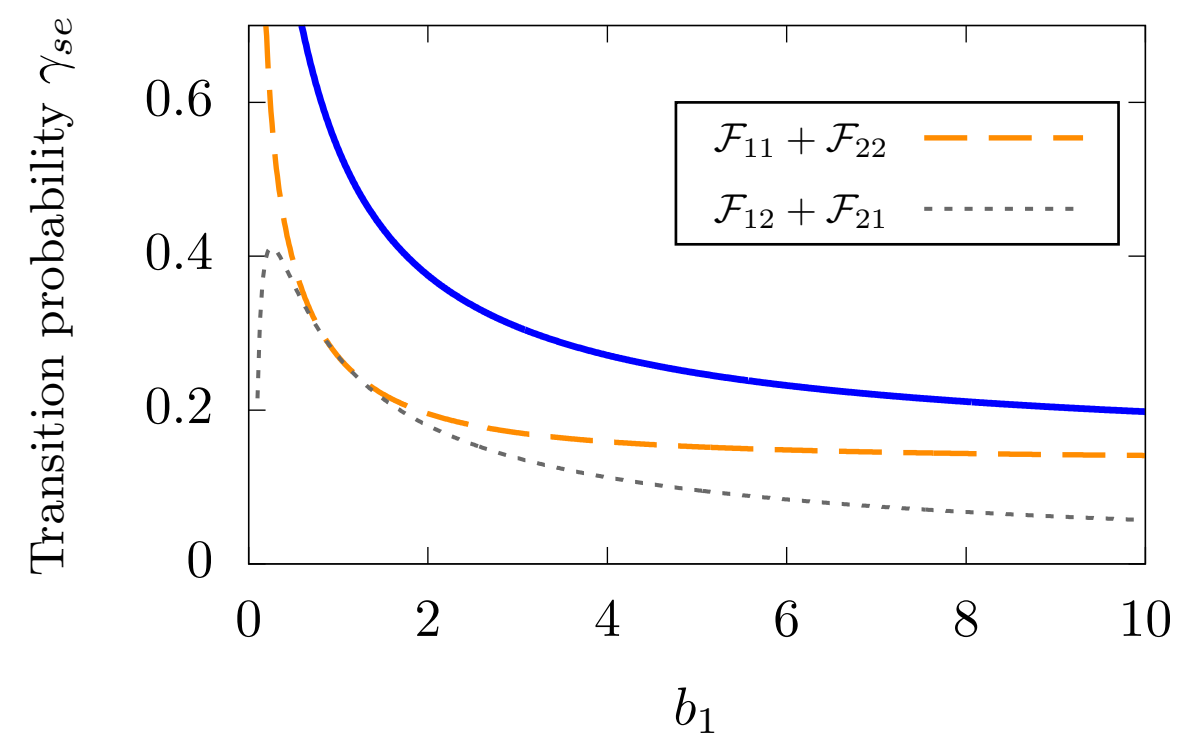

(a)

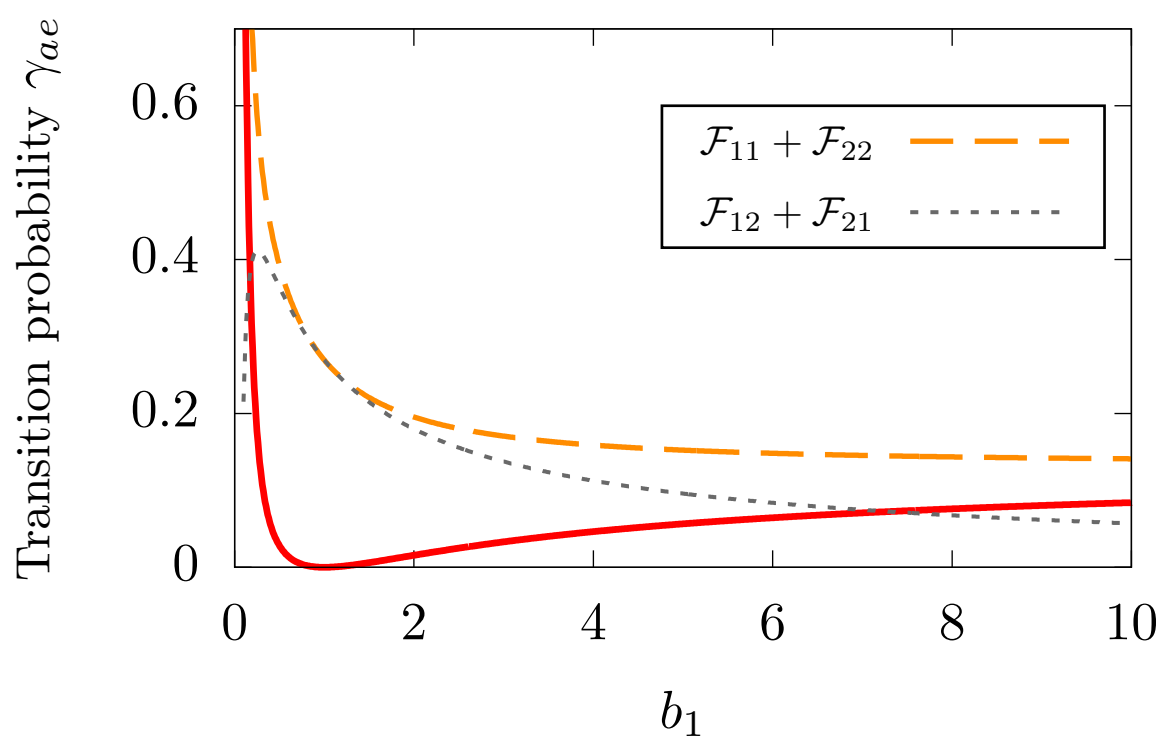

(b)

Figure 3. $(1+3)$ dimensions: (a) The transition probability from the symmetric state to the collective excited state denoted by the solid blue line. (b) The transition probability from the anti-symmetric state to the collective excited state denoted by the solid red line. In both of the cases $b_{2}=1$ is kept fixed and $b_{1}$ is varied. The orange dashed and gray dotted lines denote the contributions from $\left(\mathcal{F}_{11}+\mathcal{F}_{22}\right)$ and $\left(\mathcal{F}_{12}+\mathcal{F}_{21}\right)$ respectively. We have considered the values of the other parameters to be $\Delta E=0.1, \omega_{k}=0.1$ and $\beta=2 \pi$. 
terms of the Rindler parameters $a_{j}$ and the Rindler coordinates $\eta_{j}, \xi_{j}$. To evaluate the transition coefficients using these Green's functions one has to move to the proper time and proper acceleration which will be quite convoluted in this case. On the other hand, one can approach this issue in a more straight forward manner, where the convenient way is to keep these quantities in terms of $a_{j}, \eta_{j}$, and $\xi_{j}$ as they are. Then consider the same system of two accelerated observers in a different manner so that the transformation from these coordinates to the proper time and acceleration follows easily. In this regard we consider the parameters are different $a_{1} \neq a_{2}$ corresponding to the two observers. The proper accelerations and proper times are given by $b_{j}=a_{j} e^{-a_{j} \xi_{j}}$ and $\tau_{j}=\eta_{j} e^{a_{j} \xi_{j}}$, respectively. Now as one considers $\xi_{j}=0$ the proper accelerations and proper times become $b_{j}=a_{j}$ and $\tau_{j}=\eta_{j}$. Then for observers with equal Rindler time, the proper times are also equal (a discussion on this has been presented in appendix D). We have seen from our previous analysis that the exact form of the proportionality constant in the relation between the proper times has no role in the transition probabilities. Therefore, it should not be absurd to take the previous consideration of equal proper time.

We are going to utilize these above mentioned considerations to obtain the transition probabilities of eq. (2.8) for two accelerated atoms immersed in a thermal bath considering the Rindler modes. Then one can use $\Delta \xi_{j l}=0$ and $\Delta \eta_{j l}=\Delta \tau_{j l}=\tau_{j, 2}-\tau_{l, 1}$ in the Green's function of eq. (4.9) and (4.16). It should be noted that these Green's functions here are time translation invariant. Then one may perform the integration in eq. (2.10) by switching to the coordinates, $u_{j l}=\tau_{j, 2}-\tau_{l, 1}$ and $v_{j l}=\tau_{j, 2}+\tau_{l, 1}$. After dividing the transition coefficients by, $\left(\mu^{2} \int_{-\infty}^{\infty} d v_{j l}\right)$ one can get the response functions, which signify the transition probabilities per unit time, as

$$
R_{j l}(\Delta E)=\int_{-\infty}^{\infty} d u_{j l} e^{-i u_{j l} \Delta E} G_{\beta_{R}}^{+}\left(u_{j l}\right)
$$

In the subsequent analysis we shall use the above one here to find the transition probabilities.

\section{$6.1(1+1)$ dimensions}

In $(1+1)$ dimensions using the Green's function from eq. (4.9) we evaluate these response functions (6.1) and get

$$
\begin{array}{r}
R_{j l}(\Delta E)=\int_{0}^{\infty} \frac{d \omega_{k}}{4 \omega_{k} \sqrt{\sinh \frac{\pi \omega_{k}}{a_{j}} \sinh \frac{\pi \omega_{k}}{a_{l}}}}\left[\delta\left(\omega_{k}-\Delta E\right)\left\{\frac{e^{-\frac{\pi \omega_{k}}{2}\left(\frac{1}{a_{j}}+\frac{1}{a_{l}}\right)}}{1-e^{-\beta \omega_{k}}}+\frac{e^{\frac{\pi \omega_{k}}{2}\left(\frac{1}{a_{j}}+\frac{1}{a_{l}}\right)}}{e^{\beta \omega_{k}}-1}\right\}\right. \\
\left.+\delta\left(\omega_{k}+\Delta E\right)\left\{\frac{e^{\frac{\pi \omega_{k}}{2}\left(\frac{1}{a_{j}}+\frac{1}{a_{l}}\right)}}{1-e^{-\beta \omega_{k}}}+\frac{e^{-\frac{\pi \omega_{k}}{2}\left(\frac{1}{a_{j}}+\frac{1}{a_{l}}\right)}}{e^{\beta \omega_{k}}-1}\right\}\right] \cdot \quad(6.2)
\end{array}
$$

Here $\delta(x-a)$ denotes the Dirac delta distribution. In both of our considered transitions from the symmetric and anti-symmetric entangled states to the collective excited or the 
ground state the transition energy $\Delta E>0$. In the previous equation $\omega_{k}$ could only take positive values. Then from eq. (6.2) considering $\Delta E>0$ we get

$$
R_{j l}(\Delta E)=\frac{1}{4 \Delta E \sqrt{\sinh \frac{\pi \Delta E}{a_{j}} \sinh \frac{\pi \Delta E}{a_{l}}}}\left[\frac{e^{-\frac{\pi \Delta E}{2}\left(\frac{1}{a_{j}}+\frac{1}{a_{l}}\right)}}{1-e^{-\beta \Delta E}}+\frac{e^{\frac{\pi \Delta E}{2}\left(\frac{1}{a_{j}}+\frac{1}{a_{l}}\right)}}{e^{\beta \Delta E}-1}\right]
$$

For $j=l=1$ we get the expression of the response function $R_{11}(\Delta E)$ to be

$$
R_{11}(\Delta E)=\frac{1}{2 \Delta E}\left[\frac{1}{e^{\beta \Delta E}-1} \frac{1}{1-e^{\frac{-2 \pi \Delta E}{a_{1}}}}+\frac{1}{1-e^{-\beta \Delta E}} \frac{1}{e^{\frac{2 \pi \Delta E}{a_{1}}}-1}\right] .
$$

Note that, contrary to Minkowski mode analysis, here $R_{11}$, which is regarded as the single detector's response function, is symmetric under the exchange $\beta \leftrightarrow(2 \pi) / a_{1}$. This probably provides a justification in replacing thermal bath by a uniformly accelerated observer with respect to Rindler mode (with Unruh operators), rather than Minkowski mode.

With the help of these response functions one can obtain the transition probabilities per unit time $\gamma_{s e}^{R}$ and $\gamma_{a e}^{R}$, between different atomic states in a similar manner as done in eq. (5.9) and (5.10), as

$$
\gamma_{s e}^{R}=\left[\left\{R_{11}\left(\omega_{0}, \omega_{k}\right)+R_{22}\left(\omega_{0}, \omega_{k}\right)\right\}+\left\{R_{12}\left(\omega_{0}, \omega_{k}\right)+R_{21}\left(\omega_{0}\right), \omega_{k}\right\}\right],
$$

and

$$
\gamma_{a e}^{R}=\left[\left\{R_{11}\left(\omega_{0}, \omega_{k}\right)+R_{22}\left(\omega_{0}, \omega_{k}\right)\right\}-\left\{R_{12}\left(\omega_{0}, \omega_{k}\right)+R_{21}\left(\omega_{0}, \omega_{k}\right)\right\}\right] .
$$

These have been depicted in figure 4 .

It should be noted that we have ignored the $1 / 2$ factor coming from the expectation value of the monopole moments, as it will not affect any qualitative prediction. It should also be mentioned that these transition probabilities (6.5) are qualitatively different from the previous ones (5.9) and (5.10). In the previous case the transition probability corresponded to certain mode frequency whereas here it corresponds to unit time.

From this figure, one can observe that the transition probability for the transition from the symmetric state to the collective excited state has no anti-Unruh effect for the considered values of the fixed parameters. For the transition from the anti-symmetric state to the collective excited state, there is a visible occurrence of the anti-Unruh effect for the same considered values of the fixed parameters. We will again take up this issue in the next section.

\section{$6.2(1+3)$ dimensions}

In a similar fashion as done in the $(1+1)$ dimensional case we consider the Green's function from eq. (4.16), which corresponds to two accelerated atoms in a thermal bath described with respect to the Rindler modes. Using this Green's function we evaluate the response 


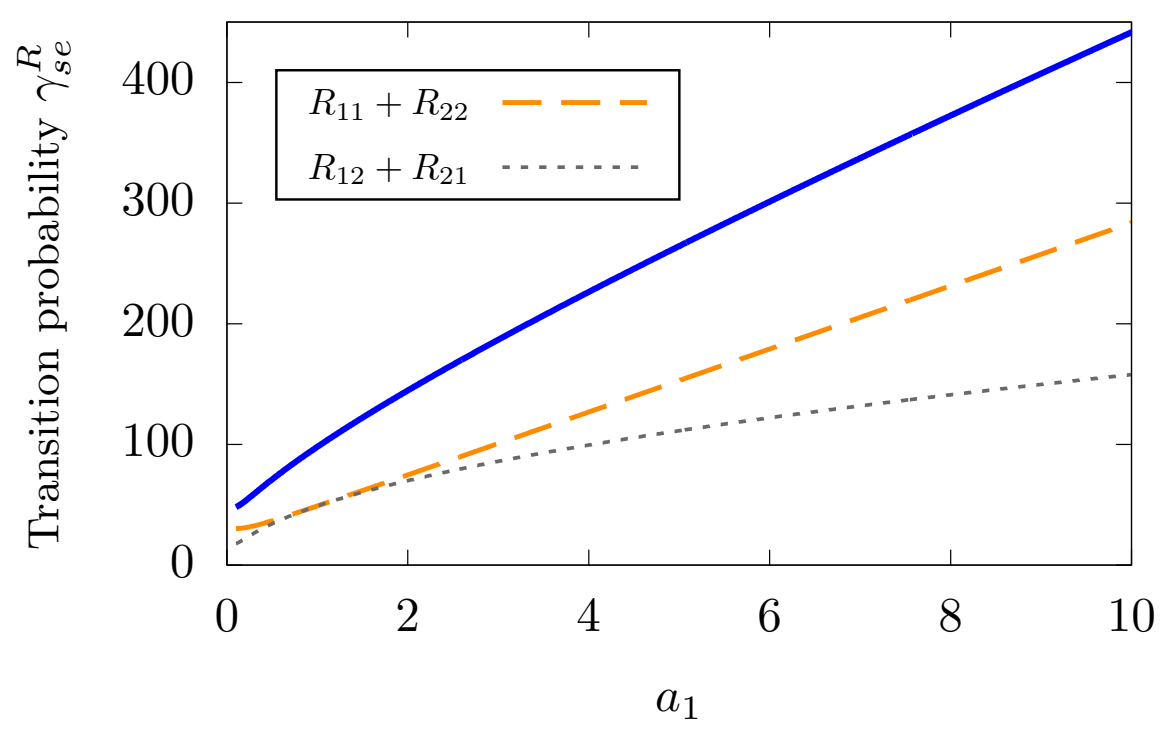

(a)

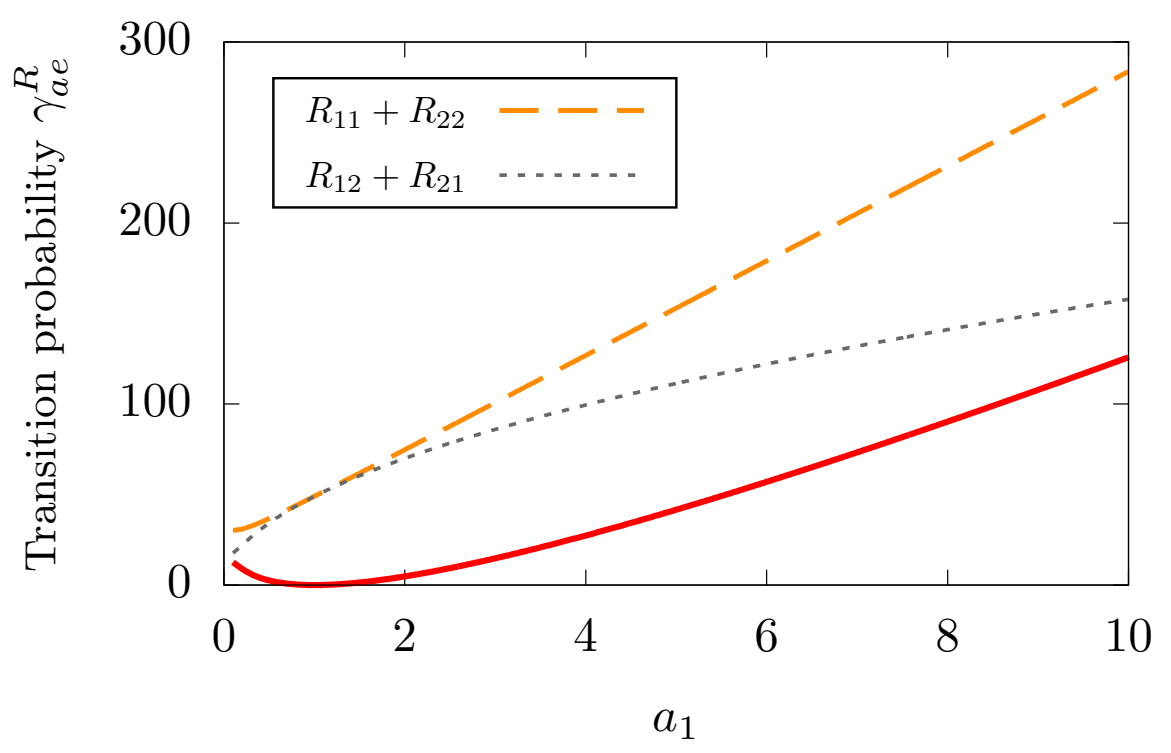

(b)

Figure 4. $(1+1)$ dimensions: (a) The transition probability from the symmetric state to the collective excited state for two observers with the Rindler modes, immersed in a thermal bath. The transition probability is denoted by solid blue line. (b) The transition probability from the antisymmetric state to the collective excited state for two observers with the Rindler modes, immersed in a thermal bath, denoted by solid red line. In both of the cases $a_{2}=1$ is kept fixed and $a_{1}$ is varied. The other parameters are $\Delta E=0.1$, and $\beta=2 \pi$. The orange dashed and gray dotted lines denote the contributions from $\left(R_{11}+R_{22}\right)$ and $\left(R_{12}+R_{21}\right)$ respectively. 
functions of eq. (6.1) and get

$$
\begin{aligned}
R_{j l}(\Delta E)= & \int_{0}^{\infty} d \omega \int \frac{d^{2} k_{p}}{(2 \pi)^{3}} \frac{2}{\sqrt{a_{j} a_{l}}}\left[\delta(\omega-\Delta E)\left\{\frac{e^{-\frac{\pi \omega}{2}\left(\frac{1}{a_{j}}+\frac{1}{a_{l}}\right)}}{1-e^{-\beta \omega}}+\frac{e^{\frac{\pi \omega}{2}\left(\frac{1}{a_{j}}+\frac{1}{a_{l}}\right)}}{e^{\beta \omega}-1}\right\}\right. \\
& \left.+\delta(\omega+\Delta E)\left\{\frac{e^{\frac{\pi \omega}{2}\left(\frac{1}{a_{j}}+\frac{1}{a_{l}}\right)}}{1-e^{-\beta \omega}}+\frac{e^{-\frac{\pi \omega}{2}\left(\frac{1}{a_{j}}+\frac{1}{a_{l}}\right)}}{e^{\beta \omega}-1}\right\}\right] \\
& \times \mathcal{K}\left[\frac{i \omega}{a_{j}}, \frac{\left|k_{p}\right|}{a_{j}}\right] \mathcal{K}\left[\frac{i \omega}{a_{l}}, \frac{\left|k_{p}\right|}{a_{l}}\right],
\end{aligned}
$$

where, we have considered $\xi_{j}=0$ for both of the observers. Here $\delta(x-a)$ denotes the Dirac delta distribution. From this eq. (6.6) considering $\Delta E>0$ we get

$$
\begin{aligned}
R_{j l}(\Delta E)= & \frac{2}{(2 \pi)^{2} \sqrt{a_{j} a_{l}}}\left[\frac{e^{-\frac{\pi \Delta E}{2}\left(\frac{1}{a_{j}}+\frac{1}{a_{l}}\right)}}{1-e^{-\beta \Delta E}}+\frac{e^{\frac{\pi \Delta E}{2}\left(\frac{1}{a_{j}}+\frac{1}{a_{l}}\right)}}{e^{\beta \Delta E}-1}\right] \\
& \times \int_{0}^{\infty} k_{p} d k_{p} \mathcal{K}\left[\frac{i \Delta E}{a_{j}}, \frac{\left|k_{p}\right|}{a_{j}}\right] \mathcal{K}\left[\frac{i \Delta E}{a_{l}}, \frac{\left|k_{p}\right|}{a_{l}}\right] .
\end{aligned}
$$

The expression of the response function $R_{11}(\Delta E)$ is obtained from this equation with $j=l=1$ as

$$
R_{11}(\Delta E)=\frac{\Delta E}{2 \pi}\left[\frac{1}{e^{\beta \Delta E}-1} \frac{1}{1-e^{\frac{-2 \pi \Delta E}{a_{1}}}}+\frac{1}{1-e^{-\beta \Delta E}} \frac{1}{e^{\frac{2 \pi \Delta E}{a_{1}}}-1}\right],
$$

which signifies the contribution of a single detector accelerated in a thermal bath described in terms of the Rindler modes. This also exhibits the $\beta \leftrightarrow(2 \pi) / a_{1}$ symmetry.

With the help of the response functions of eq. (6.7) and using eq. (6.5), one can obtain the transition probabilities per unit time $\gamma_{s e}^{R}$ and $\gamma_{a e}^{R}$, between different atomic states in $(1+3)$ dimensions. These have been depicted in figure 5 .

Note that the features of the transition probabilities are identical to $(1+1)$ dimensional case. For both dimensions, $\gamma_{s e}^{R}$ shows no anti-Unruh effect while $\gamma_{a e}^{R}$ contains anti-Unruh phenomenon. This similarity was not there in Minkowski mode analysis. Furthermore, the expressions of the response functions (6.4) and (6.8) corresponding to a single detector with Rindler modes correctly provides those for an accelerated detector [61] in a zero temperature (i.e., $\beta \rightarrow \infty$ limit) Minkowski background. Whereas that is not apparent in the case with the Minkowski modes (see eq. (5.8) and (5.16)). Such consistency probably indicates a preference of choosing Rindler modes (with Unruh operators) over Minkowski ones in mimicking thermal behaviour by accelerated observer. But to be concrete, further investigations are needed.

\section{Detailed investigation of observed anti-Unruh(-like) phenomenon}

In our previous discussions we observed that several transition probabilities are decreasing with the increase of acceleration of the observer within a particular range. It is usually, 


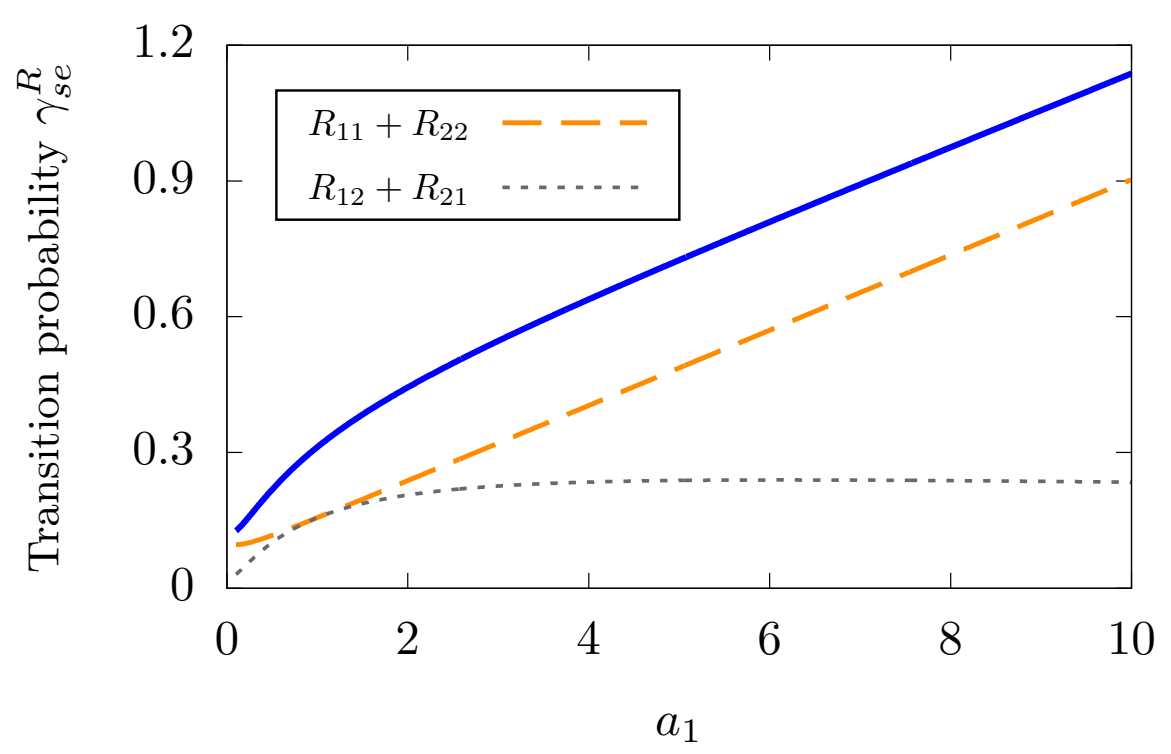

(a)

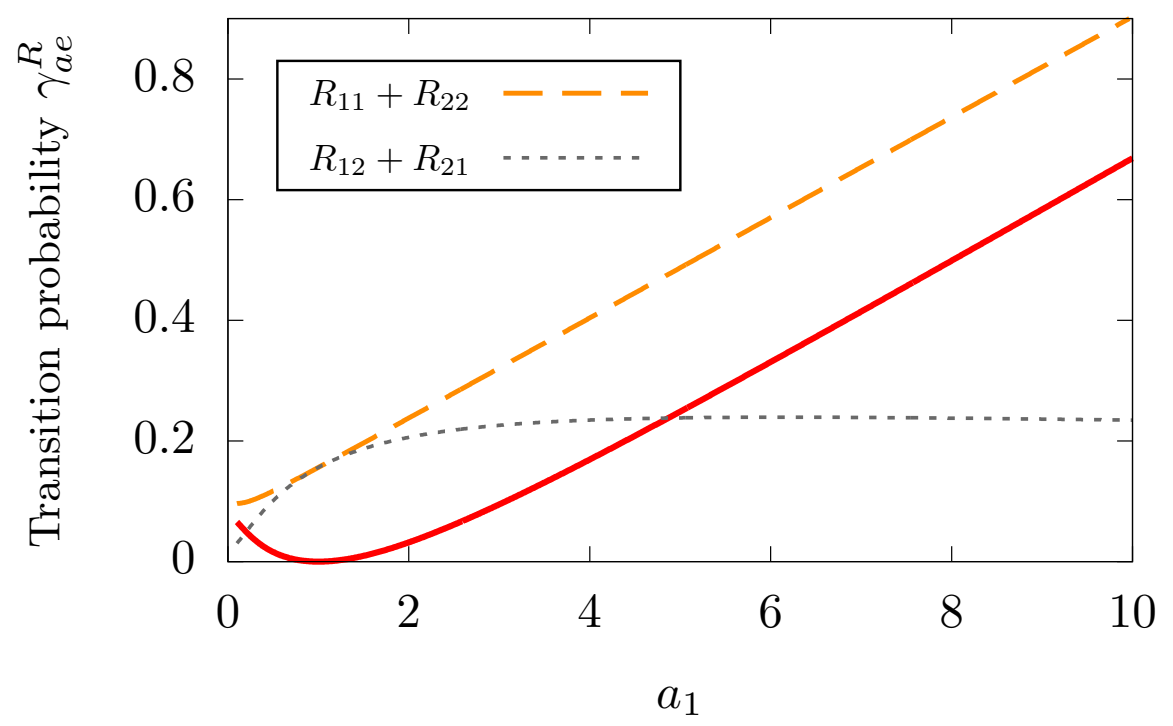

(b)

Figure 5. $(1+3)$ dimensions: (a) The transition probability from the symmetric state to the collective excited state denoted by the solid blue line. (b) The transition probability from the antisymmetric state to the collective excited state denoted by the solid red line. In both of the cases the Rindler modes are considered. In both of the cases $a_{2}=1$ is kept fixed and $a_{1}$ is varied. The value of the other parameters are $\Delta E=0.1$, and $\beta=2 \pi$. 
as observed earlier in $[48,49]$ for a different situation, called as anti-Unruh effect. In this section, the observed anti-Unruh(-like) effect for our model in the previous sections, will be further investigated in the light of required conditions for the same. In particular, we observed that for accelerated detectors in thermal bath considering the Minkowski modes there are anti-Unruh-like effects in $(1+1)$ and $(1+3)$ dimensions for both transitions from the symmetric and anti-symmetric states to the collective excited state. On the other hand, considering the same setup in terms of the Rindler modes with Unruh operators in $(1+1)$ and $(1+3)$ dimensions we observed that there is no anti-Unruh effect for the transition from the symmetric state to the collective excited state for the similar set of fixed parameters as the Minkowski modes. However, we observed that there is anti-Unruh effect for the transition from the anti-symmetric state to the collective excited state for the same values of the fixed parameters. Below we study them thoroughly and balustrade these facts by verifying the consistency with the required anti-Unruh conditions.

\subsection{Anti-Unruh effect: the conditions}

Let us first briefly summarise the conditions of anti-Unruh effect. In article [48] it was first shown that for short times the transition probability of an accelerated particle detector decreases with increasing acceleration, a phenomena better known as the anti-Unruh effect from then. In subsequent article [49] by the same authors the existence of the anti-Unruh effect for infinite time was also confirmed. The statement of the anti-Unruh effect goes like, "a uniformly accelerated particle detector coupled to the vacuum can cool down as its acceleration increases." To mathematically realize the existence of the anti-Unruh effect, done in [49], there are two particular conditions which have to be satisfied. These conditions are first mentioned below corresponding to our system of analysis.

\subsubsection{Weak anti-Unruh effect}

In our case the weak anti-Unruh effect is defined by the condition when the transition coefficients $\left(\mathcal{F}_{j l}\right)$, transition probabilities $\left(\gamma_{\omega \Omega}\right)$ or response functions $\left(R_{j l}\right)$ decrease with increasing acceleration of the atoms with all other parameters of the system fixed [49], i.e.,

$$
\partial_{b_{1}} \mathcal{F}_{j l}<0 ; \quad \partial_{b_{1}} \gamma_{\omega \Omega}<0 ; \quad \partial_{a_{1}} R_{j l}<0
$$

Note that here we have also considered taking the differentiation of the transition coefficients and response functions rather than only the transition probabilities, because these particular coefficients $\mathcal{F}_{11}$ and $R_{11}$ signify the transition probability of the single accelerated detector in thermal background. We also specify that the differentiation is taken with respect to the proper acceleration of the first detector as we have performed all of our calculations with respect to this particular frame.

\subsubsection{Strong anti-Unruh effect}

To talk about this condition a definition of excitation to de-excitation ratio (EDR) for the transition coefficients, transition probabilities and response functions is needed. The EDR 
corresponding to the transition coefficient $\mathcal{F}_{j l}(\Delta E)$, the transition probability $\gamma_{\Omega \omega}(\Delta E)$ and the response function $\mathcal{R}_{j l}(\Delta E)$ are defined as [49]

$$
\begin{aligned}
\mathcal{R}_{\mathcal{F}}(\Delta E) & =\frac{\mathcal{F}_{j l}(\Delta E)}{\mathcal{F}_{j l}(-\Delta E)} ; \\
\mathcal{R}_{\gamma}(\Delta E) & =\frac{\gamma_{\Omega \omega}(\Delta E)}{\gamma_{\Omega \omega}(-\Delta E)} ; \\
\mathcal{R}_{R}(\Delta E) & =\frac{R_{j l}(\Delta E)}{R_{j l}(-\Delta E)} .
\end{aligned}
$$

The corresponding EDR inverse temperature can be defined as [49]

$$
\mathcal{B}_{E D R}=-\frac{1}{\Delta E} \ln (\mathcal{R}) .
$$

The condition for strong anti-Unruh effect is met when the EDR temperature decreases with increasing detector acceleration. The mathematical representation of the strong antiUnruh effect is characterized by the condition of

$$
\partial_{b_{1}} \mathcal{B}_{E D R}\left(\Delta E, b_{2}, \omega_{k}\right)>0 .
$$

Below we shall check whether our observed phenomena are consistent with these mentioned conditions. This will not only provide a verification of our aforesaid claim, but also provide a classification of the anti-Unruh(-like) phenomenon. Before proceeding further, it may be noted that satisfaction of strong anti-Unruh condition implies automatic satisfaction of weak condition; while the reverse is may not be true (see [49] for details). The strong anti-Unruh effect always refers to the occurrence of the weak anti-Unruh effect unless the conditions

$$
\partial_{b_{1}} \mathbb{F}(-\Delta E)>0 \text {, and } \partial_{b_{1}} \mathbb{E}(\Delta E)>0 ;
$$

are satisfied simultaneously, see [49]. Here $\mathbb{E}(\Delta E)$ can be considered to be any of the $\mathcal{F}_{j l}(\Delta E), \gamma_{\omega \Omega}(\Delta E)$ or $R_{j l}(\Delta E)$, while for $R_{j l}(\Delta E)$ the derivative is taken with respect to $a_{1}$. The occurrence of strong anti-Unruh effect, when one of these conditions or both of them are violated signifies the definite satisfaction of the weak condition. On the other hand, when both of these conditions (7.5) are simultaneously satisfied, the satisfaction of the strong condition will not imply the satisfaction of the weak condition and in that case one cannot comment about the nature of this phenomena. We shall check both the conditions from eq. (7.1) and (7.4), and accordingly categorise the phenomenon as either weak or strong anti-Unruh effect.

\subsection{Case I: Minkowski mode}

\subsection{1 (1 + 1)-dimensions}

We have seen from our analysis that in the system of two entangled atoms accelerated in a thermal background, considering the $(1+1)$ dimensions and Minkowski modes, as one estimates the transition probability between different states one first gets a decreasing probability with increasing proper acceleration $b_{1}$, signifying anti-Unruh-like effect. Then 


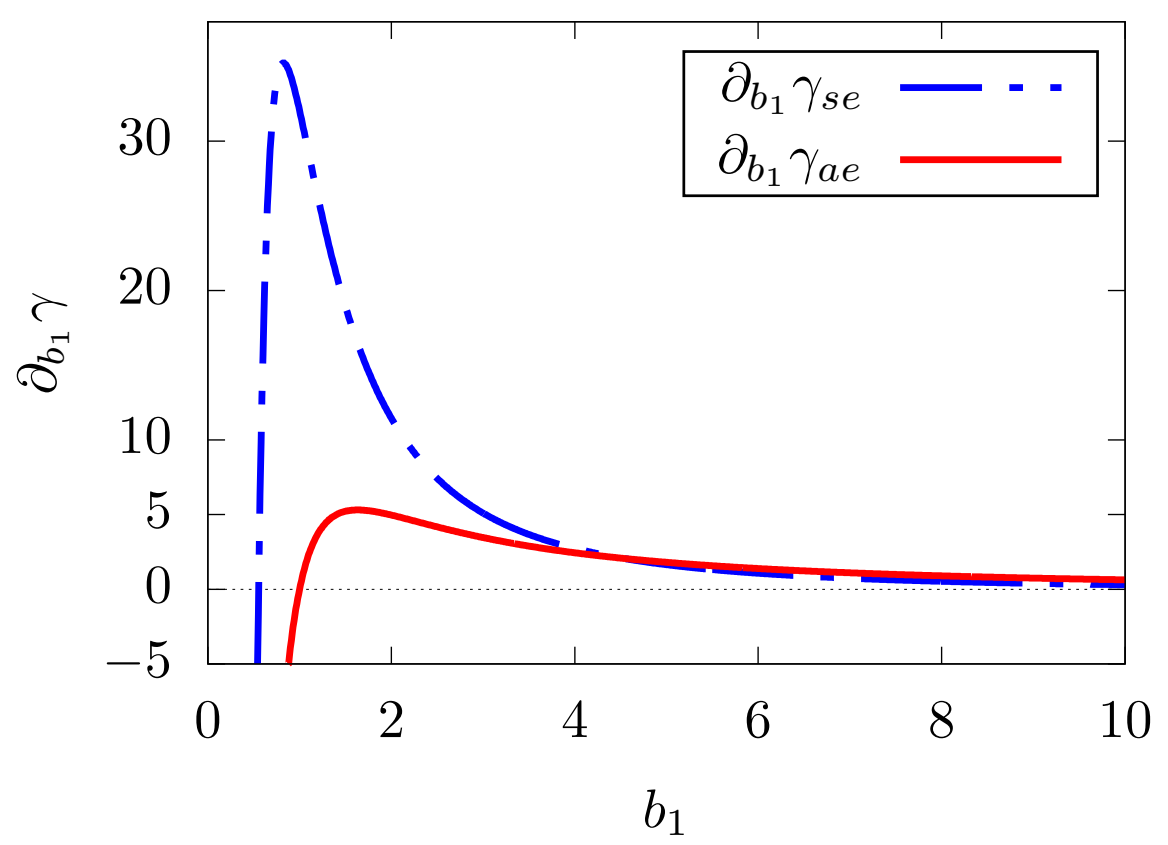

Figure 6. The quantity $\partial_{b_{1}} \gamma_{s e}$ is plotted with respect to varying $b_{1}$ for accelerated atoms in thermal background in $(1+1)$ dimensions, depicted by dash-dotted blue line. The quantity $\partial_{b_{1}} \gamma_{a e}$ is plotted with respect to varying $b_{1}$ for accelerated atoms in thermal background in $(1+1)$ dimensions, depicted by red line.

one gets an increasing transition probability with increasing acceleration $b_{1}$ signifying the Unruh-like effect. These phenomena can be understood from figure 2. Here we are going to check whether the transition coefficients and transition probabilities satisfies the weak and strong conditions of the so called anti-Unruh effect. After analyzing the results we shall like to predict some possible source of origin behind it.

First, to check the weak condition for certain values of the parameters $\omega_{k}=0.1$, $\Delta E=0.1$, and $b_{2}=1$ we plot $\partial_{b_{1}} \gamma_{\omega \Omega}$ VS $b_{1}$ in figure 6 corresponding to transitions from the symmetric and anti-symmetric states to the collective excited state (here we take the same values of parameters as taken in figure 2 in order to have a proper comparison).

These plots are meant to provide confirmation in support of the existence of the weak anti-Unruh effect when the functions have negative values. We observe that this is the case in the lower regimes of the proper acceleration $b_{1}$ - in the case of $\gamma_{s e}$ for $b_{1}$ less than 0.5 and in the case of $\gamma_{a e}$ for $b_{1}$ less than 1 . Note that these are the values of $b_{1}$ up to which the transition probabilities were decreasing (see figure 2).

Now to check whether this is complied with strong condition, in figure 7 we have plotted the differentiation of EDR inverse temperature with respect to $b_{1}$ for the same two transitions. Note that for both the cases $\partial_{b_{1}} \mathcal{B}_{E D R}$ are always negative throughout the range of $b_{1}$ and so it does not satisfy the strong condition. Hence here we have weak anti-Unruh effect.

Now the question arises what is the origin of this anti-Unruh-like phenomena in these transition probabilities $\gamma_{s e}$ and $\gamma_{a e}$ ? Is it happening solely because of the entanglement 


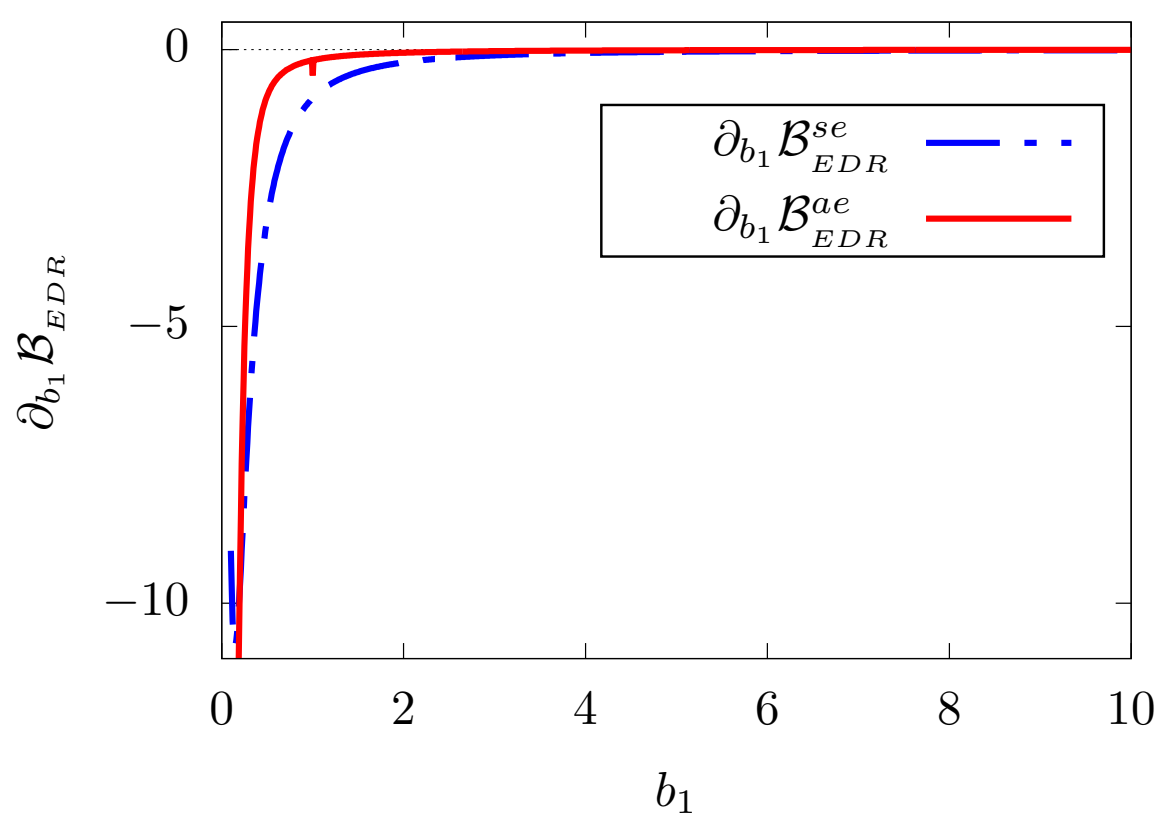

Figure 7. The quantity $\partial_{b_{1}} \beta_{\gamma_{s e}}$ is plotted with respect to varying $b_{1}$, depicted by dash-dotted blue line. The quantity $\partial_{b_{1}} \beta_{\gamma_{a e}}$ is plotted with respect to varying $b_{1}$, depicted by red line.

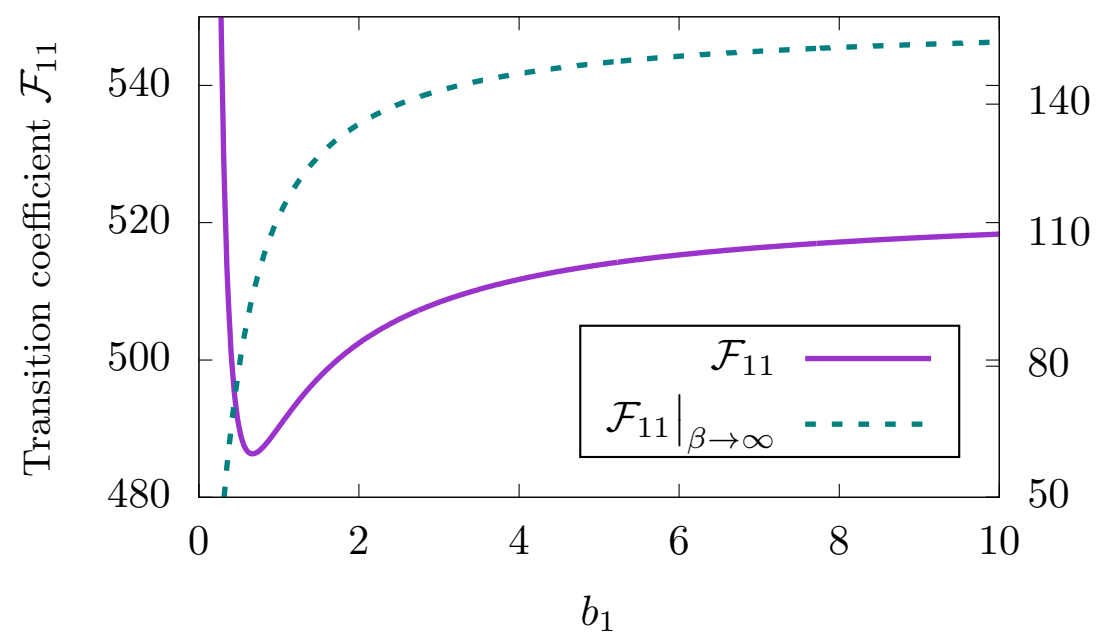

Figure 8. The transition coefficient $\mathcal{F}_{11}(\Delta E)$ with respect to varying $b_{1}$ for accelerated atoms in thermal background considering Minkowski modes. The solid violet line with the left vertical axis denotes this particular case. The transition coefficient $\mathcal{F}_{11}(\Delta E)$ in the same scenario with the temperature of the thermal bath now zero denoted by the dotted green line with right vertical axis.

between the atoms? In this regard, we want to mention that this phenomena is not only visualized in $\gamma_{s e}$ and $\gamma_{a e}$, but also in the transition coefficient $\mathcal{F}_{11}(\Delta E)$, figure 8.

It is to be noted that the transition coefficient $\mathcal{F}_{11}(\Delta E)$ signifies the situation when a single detector is accelerated in a thermal bath. In that case the entanglement do not 


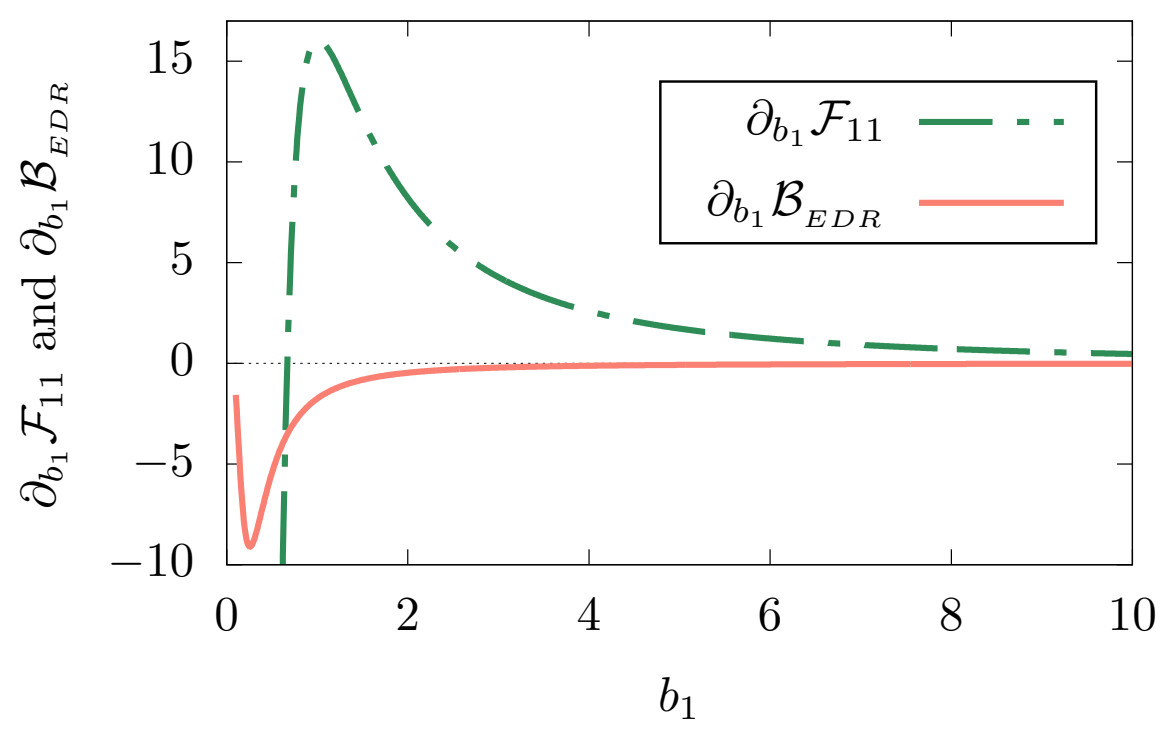

Figure 9. Differentiation of the transition coefficient $\mathcal{F}_{11}(\Delta E)$ with respect to $b_{1}$ plotted against varying $b_{1}$ for accelerated atoms in thermal background, denoted by the dash-dotted green line. The negative value of this quantity $\partial_{b_{1}} \mathcal{F}_{11}(\Delta E)$ gives the condition for weak anti-Unruh effect. Here the quantity $\partial_{b_{1}} \beta_{\mathcal{F}_{11}}$ is also plotted with respect to varying $b_{1}$ and it is denoted by the solid violet line. The positive value of this quantity gives the strong condition for the anti-Unruh-like effect.

play any role into the picture and one can assert that the source of the anti-Unruh-like effect is not from entanglement, at least in this case. Then the attention is bound to be shifted towards the effects of the thermal bath as a possible origin of the anti-Unruh-like phenomena. In this regard, in the same figure 8 we have also plotted the $\mathcal{F}_{11}(\Delta E)$ in the limit of $\beta \rightarrow \infty$ or for zero temperature of the thermal bath. Interestingly it shows no anti-Unruh-like effect, which suggests one reason behind the anti-Unruh-like effect to be the non zero temperature of the thermal bath in which the atoms are accelerating.

Subsequently, we have studied the nature of the anti-Unruh-like phenomenon arising in $\mathcal{F}_{11}(\Delta E)$. We can observe that the transition coefficient $\mathcal{F}_{11}(\Delta E)$ satisfies the condition for the weak anti-Unruh effect, but do not agree with the condition for the strong anti-Unruh effect (see figure 9).

\subsection{2 (1+3)-dimensions}

Next we consider the case for entangled atoms accelerated in a thermal bath as seen with respect to the Minkowski modes in a $(1+3)$ dimensional spacetime. For the same set of values of the parameters $\omega_{k}=0.1, \Delta E=0.1$, and $b_{2}=1$ as taken in figure 3 we have plotted, see figure 10 , the $\partial_{b_{1}} \gamma_{s e}$ and $\partial_{b_{1}} \gamma_{a e}$ with respect to $b_{1}$, which respectively correspond to transitions from the symmetric and anti-symmetric states to the collective excited state. For symmetric case, $\partial_{b_{1}} \gamma_{s e}$ is negative for all selected values of $b_{1}$ while for other case it is negative till $b_{1}=1$. These exactly comply with figure 3 and thereby provide confirmation in support of the satisfaction of the weak anti-Unruh condition. 


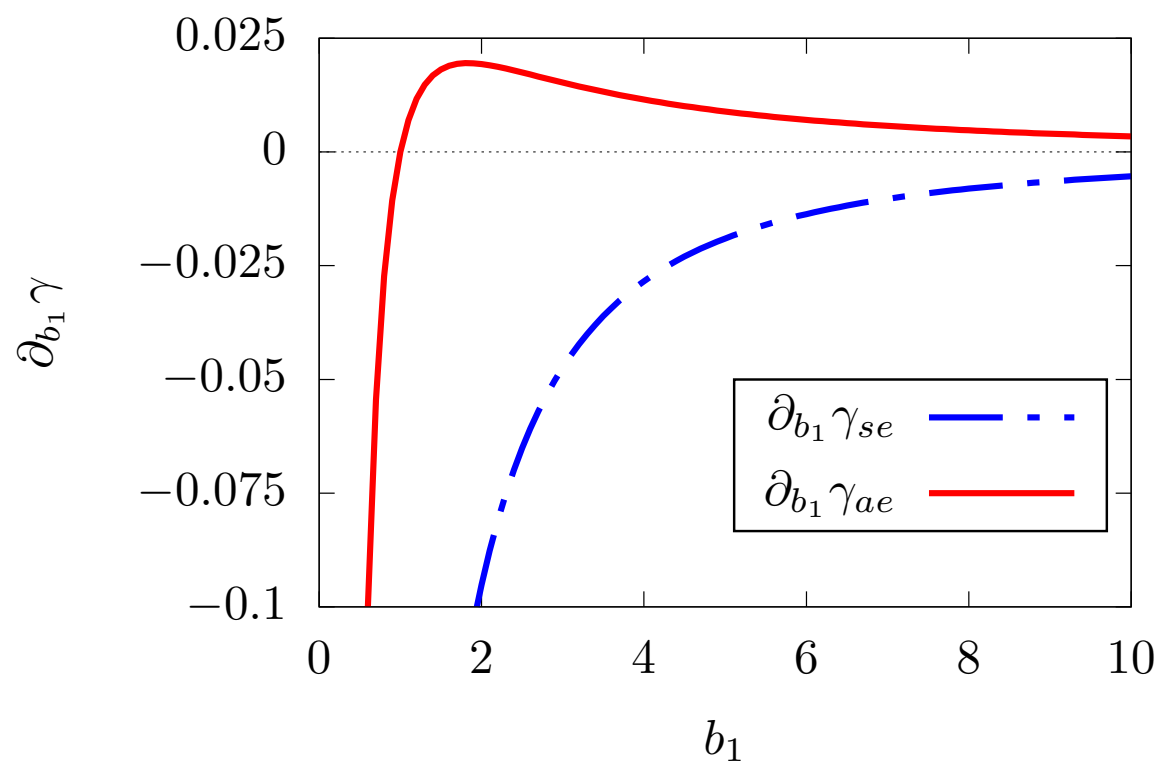

Figure 10. The quantity $\partial_{b_{1}} \gamma_{s e}$ is plotted with respect to varying $b_{1}$ for accelerated atoms in thermal background in $(1+3)$ dimensions, depicted by dash-dotted blue line. The quantity $\partial_{b_{1}} \gamma_{a e}$ is plotted with respect to varying $b_{1}$ for accelerated atoms in thermal background in $(1+3)$ dimensions, depicted by solid red line.

In figure 11 we have plotted the differentiation of EDR inverse temperature with respect to $b_{1}$ for the same two transitions, which provide the condition for the occurrence of the strong anti-Unruh effect for positive values. We observed that for the transition from the symmetric entangled state to the collective excited state the quantity $\partial_{b_{1}} \mathcal{B}_{E D R}^{s e}$ has negative value up to around $b_{1}=3$ and then it gets a small positive value and tends to decrease to zero for further increase in $b_{1}$. On the other hand, for the transition from the antisymmetric entangled state to the collective excited state the quantity $\partial_{b_{1}} \mathcal{B}_{E D R}^{a e}$ has positive value around $b_{1}=0.25$ and then it becomes negative and remains so for further increase in $b_{1}$. These analysis suggest that while the condition for weak anti-Unruh effect is satisfied for a wide range of the parameter $b_{1}$, the condition for strong anti-Unruh effect is satisfied in a much smaller range residing inside that of the weak case. Therefore, for the parameter values of $b_{1}$ when strong anti-Unruh effect is satisfied the weak anti-Unruh effect is always satisfied, consistent with our previous assertions.

Here also like the previous $(1+1)$ dimensional case we have tried to understand the origin of the anti-Unruh-like effect. In this direction in figure 12 we have plotted the transition coefficient $\mathcal{F}_{11}$ in $(1+3)$ dimensions considering the same sets of parameters. This particular transition coefficient signifies the situation if there were only one two-level atomic detector accelerating in the thermal bath. Like the $(1+1)$ dimensional case here also we have observed the anti-Unruh-like phenomenon, discarding any possibility of entanglement being the sole origin of this effect. Furthermore, in figure 13 we have studied whether the antiUnruh-like effect for $\mathcal{F}_{11}$ is of weak or strong origin. From this figure we observed that in $(1+3)$ dimensions it satisfies the condition for weak anti-Unruh effect but not the strong one. 


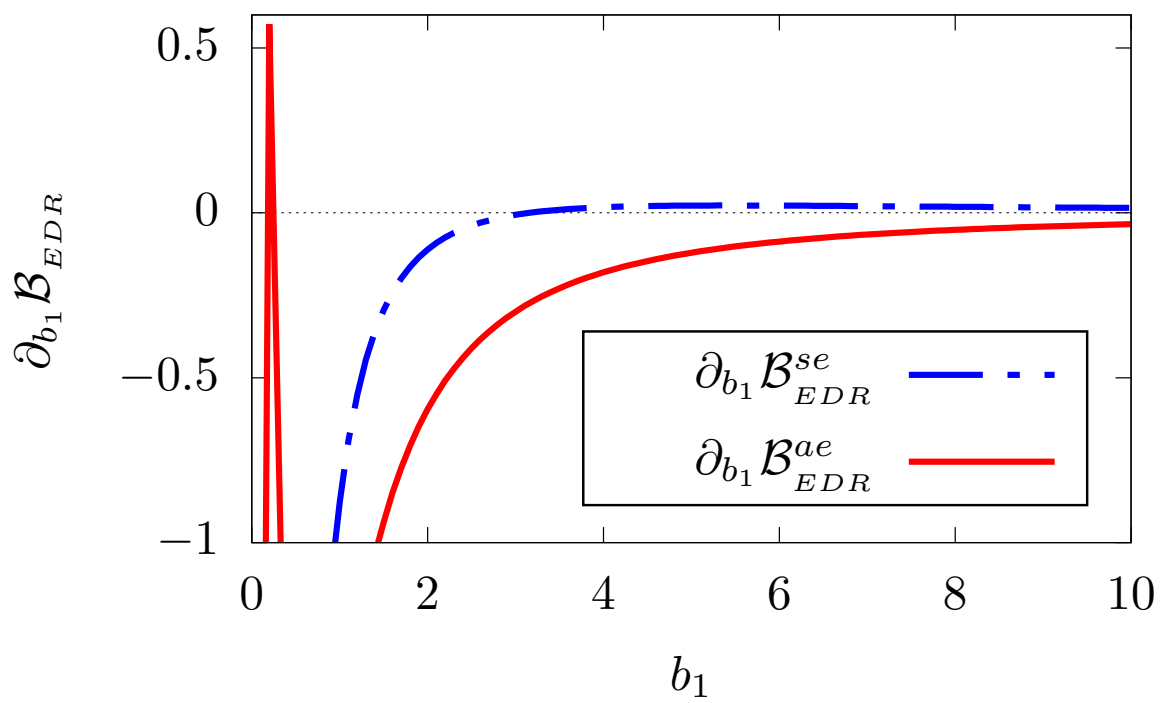

(a)

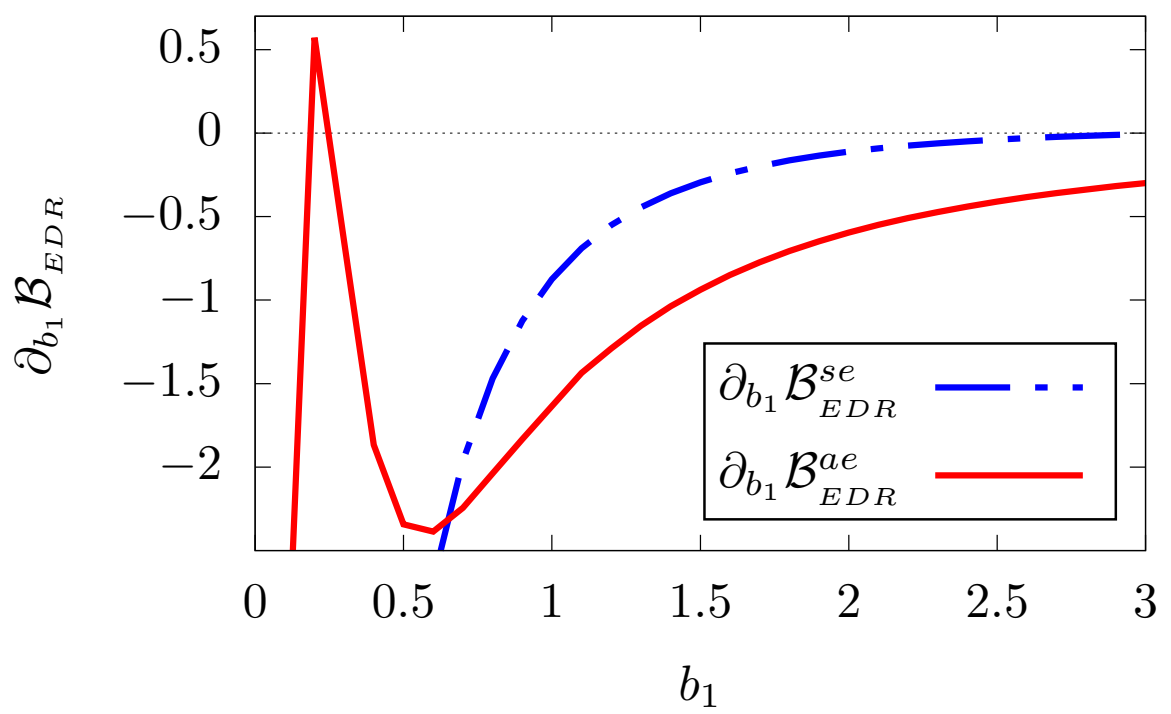

(b)

Figure 11. (a) The quantity $\partial_{b_{1}} \mathcal{B}_{E D R}^{\text {se }}$ is plotted with respect to varying $b_{1}$ for accelerated atoms in thermal background in $(1+3)$ dimensions, depicted by dash-dotted blue line. The quantity $\partial_{b_{1}} \mathcal{B}_{E D R}^{a e}$ is plotted with respect to varying $b_{1}$ for accelerated atoms in thermal background in $(1+3)$ dimensions, depicted by solid red line. (b) The same plot as depicted in the previous subfigure with the $x$ - range in the initial region now emphasized. 


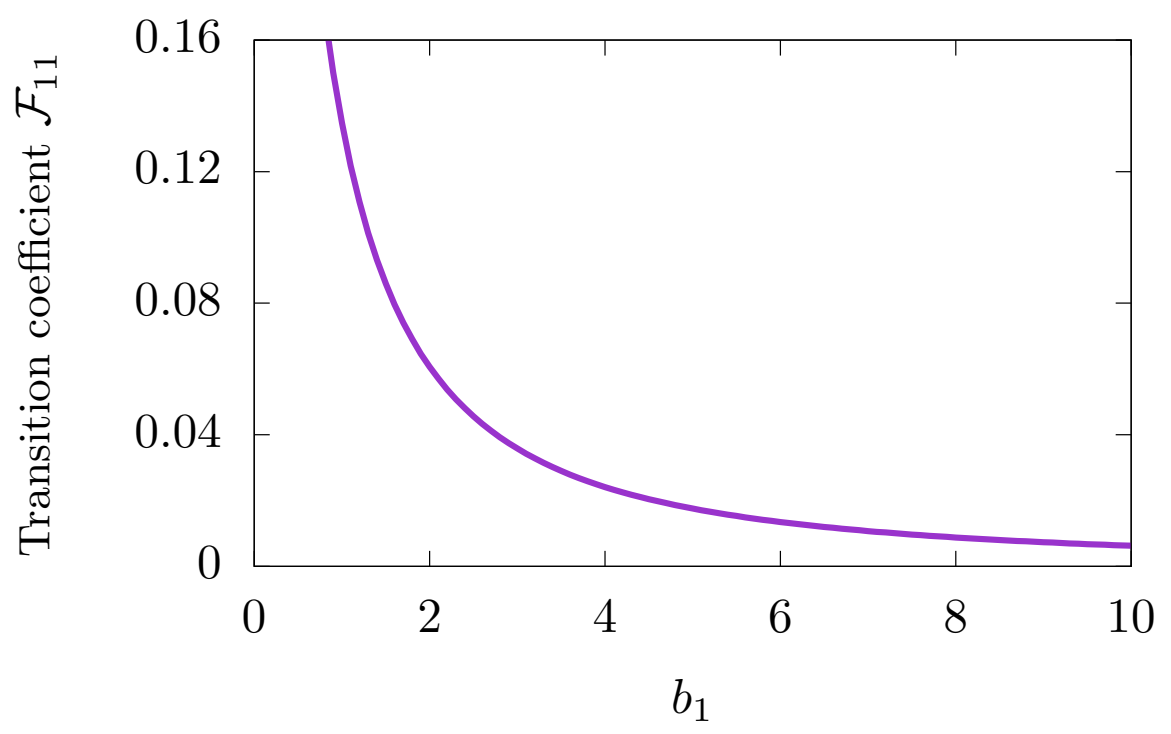

Figure 12. The transition coefficient $\mathcal{F}_{11}(\Delta E)$, plotted with respect to varying $b_{1}$ for accelerated atoms in thermal background considering Minkowski modes and $(1+3)$ dimensions.

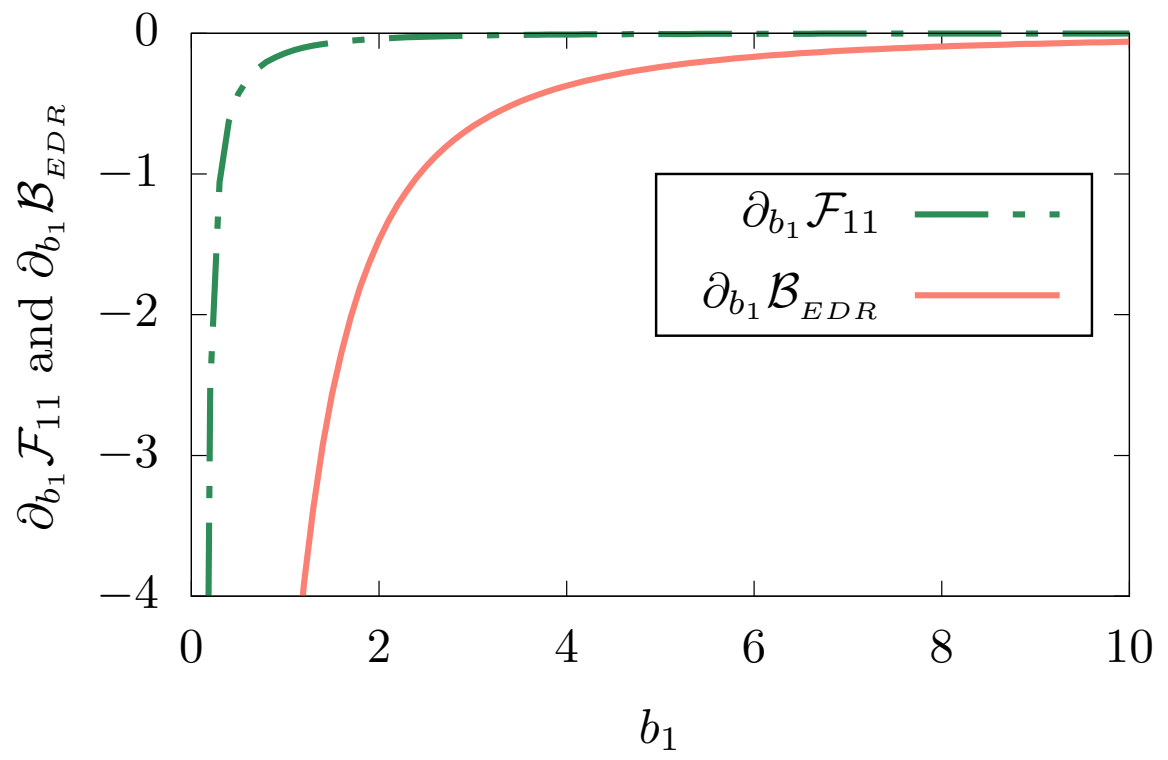

Figure 13. The derivative of the transition coefficient $\mathcal{F}_{11}(\Delta E)$, plotted with respect to varying $b_{1}$ for accelerated atoms in thermal background considering Minkowski modes and $(1+3)$ dimensions, denoted by dash-dotted green line. In this figure the quantity $\partial_{b_{1}} \mathcal{B}_{E D R}$ is also plotted, which is denoted by the solid line. 


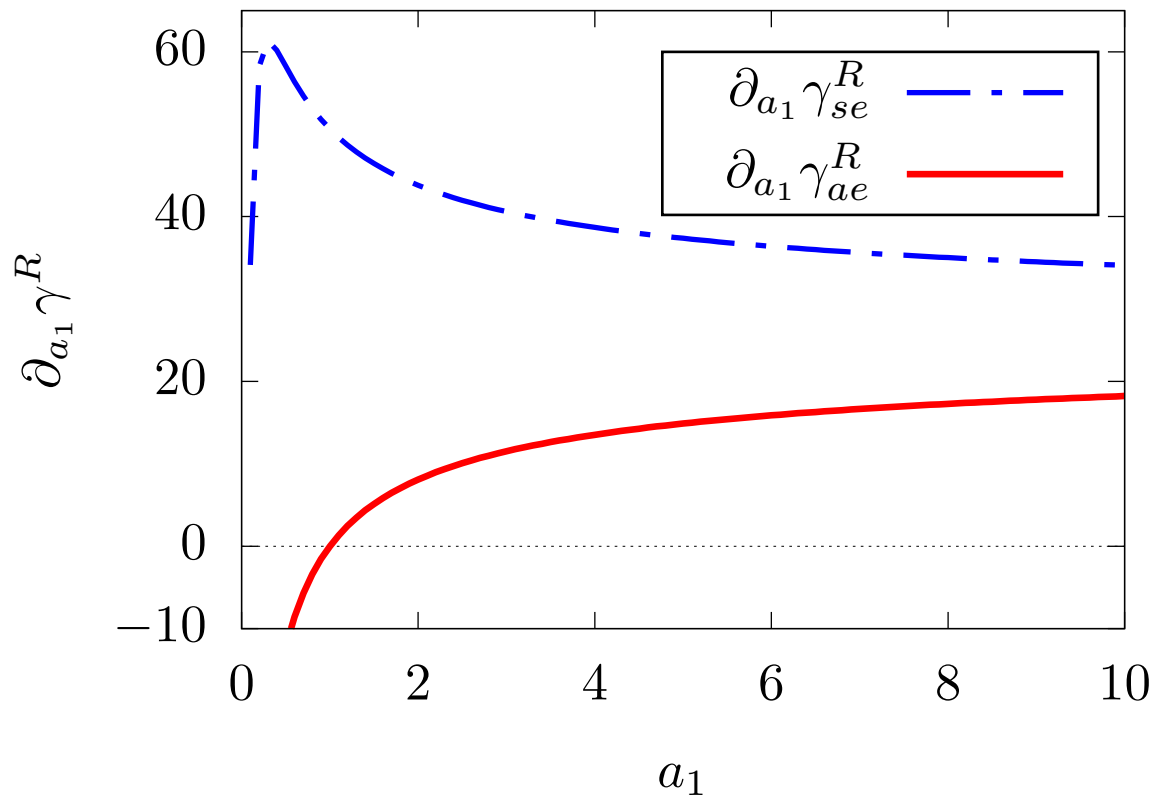

Figure 14. The quantity $\partial_{a_{1}} \gamma_{s e}^{R}$ is plotted with respect to varying $a_{1}$ for accelerated atoms in thermal background considering Rindler modes, depicted by dash-dotted blue line. The quantity $\partial_{a_{1}} \gamma_{a e}^{R}$ is plotted with respect to varying $a_{1}$ for accelerated atoms in thermal background considering Rindler modes, depicted by solid red line.

\subsection{Case II: Rindler mode}

\subsection{1 $(1+1)$-dimensions}

To understand the anti-Unruh effect for the transition probabilities considering the Rindler modes, we have plotted $\partial_{a_{1}} \gamma_{s e}^{R}$ and $\partial_{a_{1}} \gamma_{a e}^{R}$ with respect to varying $a_{1}$ in figure 14 . In obtaining this figure we have kept the other parameters fixed $\Delta E=0.1, a_{2}=1$, same as in the figure 4. We observed that for transitions from the symmetric entangled state to the collective excited state there is no weak anti-Unruh effect, confirmed from figure 14. On the other hand, for transition from the anti-symmetric state to the collective excited state there is weak anti-Unruh affect up to the value of $a_{1}=1$, complied from figure 14 .

In figure 15 we have plotted the quantity $\partial_{a_{1}} \mathcal{B}_{E D R}^{\text {se }}$ and $\partial_{a_{1}} \mathcal{B}_{E D R}^{a e}$ with respect to the acceleration $a_{1}$ considering the Rindler modes. These quantities correspond to the variation of the inverse EDR temperature for transitions from the symmetric and anti-symmetric states to the collective excited state with respect to $a_{1}$. These plots are meant to provide confirmation in support of occurrence of any strong anti-Unruh effect. We mention that while for the transition from the symmetric to excited state $\partial_{a_{1}} \mathcal{B}_{E D R}^{s e}$ is always negative and there is no sign of strong anti-Unruh effect, the case for anti-symmetric to excited state is different. In that case $\partial_{a_{1}} \mathcal{B}_{E D R}^{a e}$ is positive in the whole region compared to a smaller region where weak condition is valid. From figure 16 and figure 14 one can observe that $\partial_{a_{1}} \gamma_{a e}^{R}(-\Delta E)$ and $\partial_{a_{1}} \gamma_{a e}^{R}(\Delta E)$ are both positive for values of $a_{1}$ above $a_{1}=1$, and from eq. (7.5) it is to be noted that in that case strong anti-Unruh effect does not refer to the 


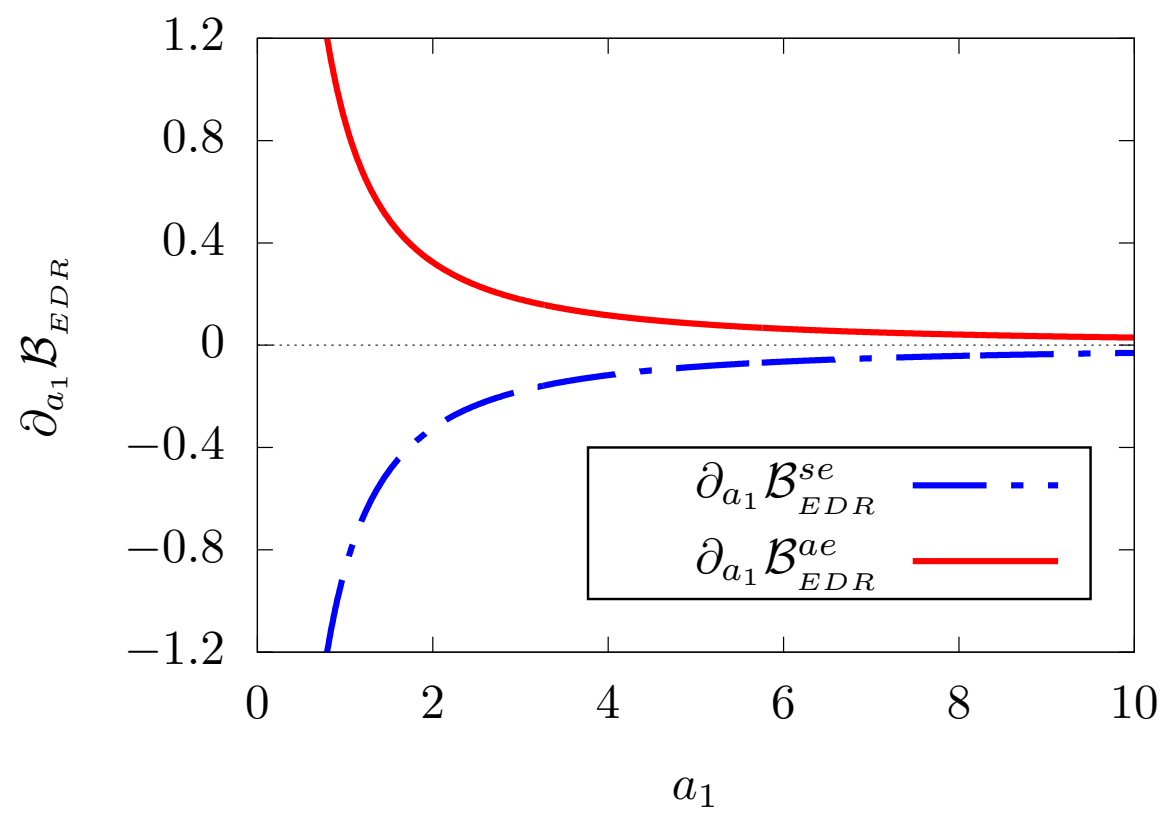

Figure 15. The quantity $\partial_{a_{1}} \mathcal{B}_{E D R}^{s e}$ is plotted with respect to varying $a_{1}$ for accelerated atoms in thermal background considering Rindler modes, depicted by dash-dotted blue line. The quantity $\partial_{a_{1}} \mathcal{B}_{E D R}^{a e}$ is plotted with respect to varying $a_{1}$ for accelerated atoms in thermal background considering Rindler modes, depicted by solid red line.

satisfaction of weak condition. Therefore, there is no shortcomings in the analysis. Then we note that the anti-Unruh effect occurring in this case is of both strong and weak nature below the value $a_{1}=1$, and above it there is no anti-Unruh effect.

Like the Minkowski mode case here also we have tried to understand the origin of the anti-Unruh effect. As discussed earlier the particular response function $\mathcal{R}_{11}(\Delta E)$ signifies the contribution of a single two-level atomic detector, accelerated in a thermal background. Therefore, we consider the contribution from the response function $R_{11}$ for $\Delta E>0$ from eq. (6.4) and observe

$$
\partial_{a_{1}} R_{11}(\Delta E)=\frac{\pi\left(e^{\beta \Delta E}+1\right) e^{\frac{2 \pi \Delta E}{a_{1}}}}{a_{1}^{2}\left(e^{\beta \Delta E}-1\right)\left(e^{\frac{2 \pi \Delta E}{a_{1}}}-1\right)^{2}},
$$

which is positive for all positive values of $\Delta E$, thus giving no anti-Unruh effect.

This response function $R_{11}(\Delta E)$ in $(1+1)$ dimensions considering the Rindler modes is plotted in figure 17, where we observed that there is no visible case of anti-Unruh effect for the same set of values of the parameters. It should be noted that this is in contrary to the case considering the Minkowski modes. Therefore, in this case entanglement must have played a significant role to provide this outcome. In fact one may plot the differentiation of the transition probability $\partial_{a_{1}} \gamma_{a e}^{R}$ with respect to $a_{1}$ in the $\beta \rightarrow \infty$ limit, the zero temperature case, to check that the anti-Unruh effect is present there too. Furthermore, in figure 18 we have checked the conditions for weak and strong anti-Unruh effect for $R_{11}(\Delta E)$ and 


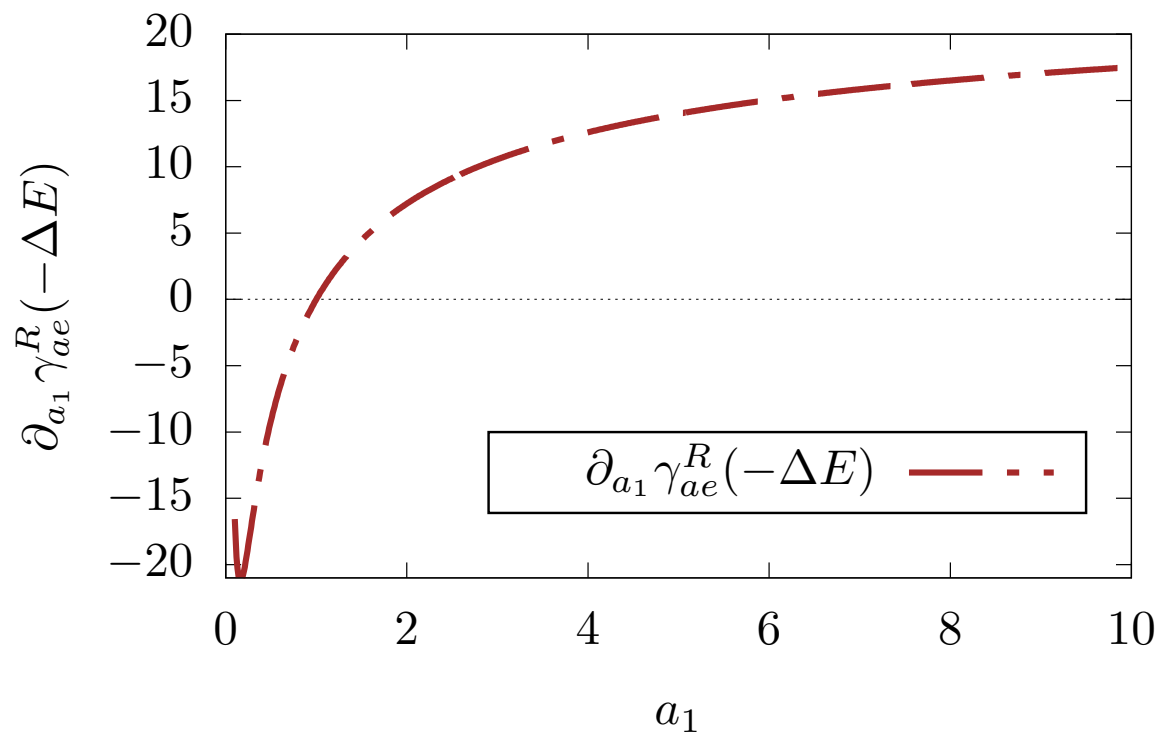

Figure 16. The quantity $\partial_{a_{1}} \gamma_{a e}^{R}(-\Delta E)$ corresponding to the transition from the anti-symmetric state to the collective excited state is plotted with respect to varying $a_{1}$ for accelerated atoms in thermal background with Rindler modes. This quantity is positive for values above $a_{1}=1$, like the $\partial_{a_{1}} \gamma_{a e}^{R}(\Delta E)$ previously depicted in figure 14 .

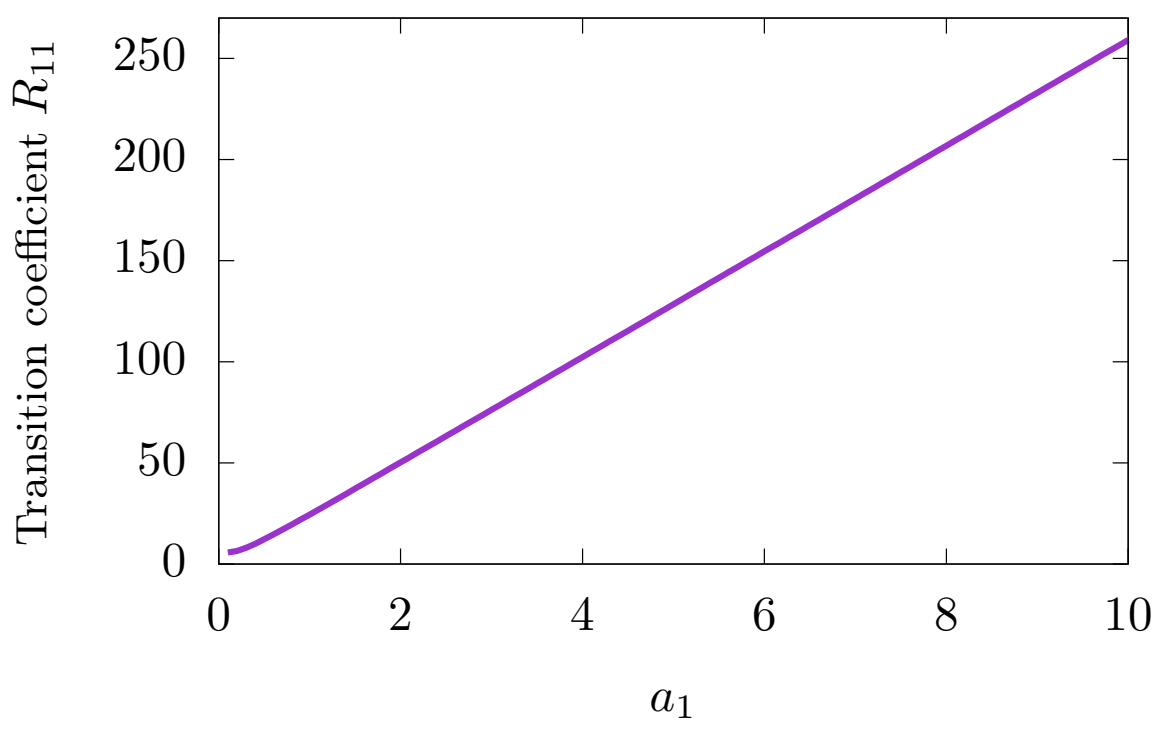

Figure 17. The response function $R_{11}(\Delta E)$, plotted with respect to varying $a_{1}$ for accelerated atoms in thermal background considering the Rindler modes in $(1+1)$ dimensions. 


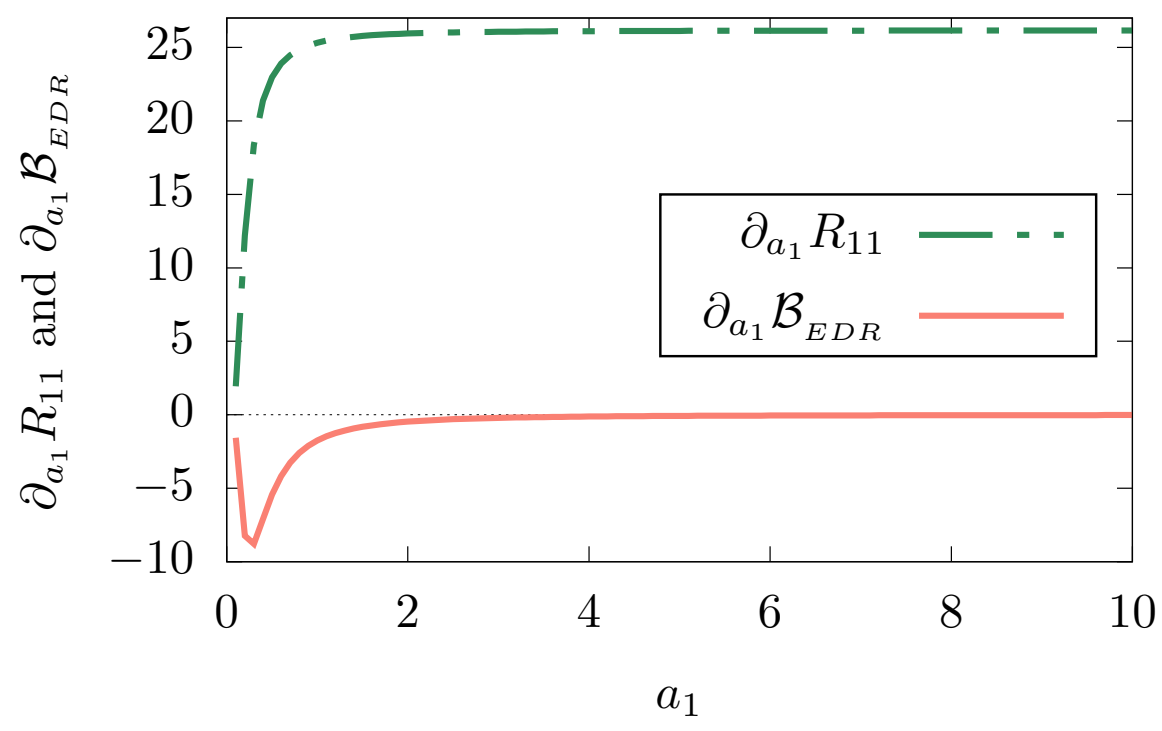

Figure 18. The differentiation of the response function $R_{11}(\Delta E)$, plotted with respect to varying $a_{1}$ for accelerated atoms in thermal background considering the Rindler modes in $(1+1)$ dimensions, denoted by dash-dotted green line. In this figure the quantity $\partial_{a_{1}} \mathcal{B}_{E D R}$ corresponding to the response function is also plotted, which is denoted by the solid line.

we confirm the absence of any anti-Unruh effect in that parameter range. This reconfirms that entanglement is crucial for anti-Unruh phenomenon in case of Rindler mode analysis.

\subsection{2 (1+3)-dimensions}

In figure 19 we have plotted $\partial_{a_{1}} \gamma_{s e}^{R}$ and $\partial_{a_{1}} \gamma_{a e}^{R}$, i.e., the derivatives of the transition probabilities corresponding to transitions from the symmetric and anti-symmetric states to the collective excited state considering the Rindler modes, with respect to varying $a_{1}$. The other parameters are kept fixed $\Delta E=0.1, a_{2}=1$, same as in the figure 5. Like earlier these plots denote the conditions for weak anti-Unruh effect. In particular, we observed that for transition from the symmetric entangled state to the collective excited state there is no weak anti-Unruh effect for these particular parameter values, see figure 19. However, for the same parameter values for the transition from the anti-symmetric state to the collective excited state there is weak anti-Unruh affect up to the value of $a_{1}=1$ also seen from figure 19 .

In figure 20 we have plotted the quantity $\partial_{a_{1}} \mathcal{B}_{E D R}^{\text {se }}$ and $\partial_{a_{1}} \mathcal{B}_{E D R}^{a e}$ with respect to the acceleration $a_{1}$ to understand the strong anti-Unruh effect in this case. These quantities correspond to the variation of the inverse EDR temperature for transitions from the symmetric and anti-symmetric states to the collective excited state with respect to $a_{1}$. These plots are meant to provide confirmation in support of the occurrence of any strong anti-Unruh effect. From this figure we observed that for both the transitions from the symmetric and antisymmetric states to the collective excited state there is no sign of strong anti-Unruh effect.

Like the previous cases here also we have tried to understand the origin of these antiUnruh effect. In this regard, we have plotted the response function $R_{11}(\Delta E)$ in $(1+3)$ dimensions considering the Rindler modes in figure 21 . Here also we observed that there 


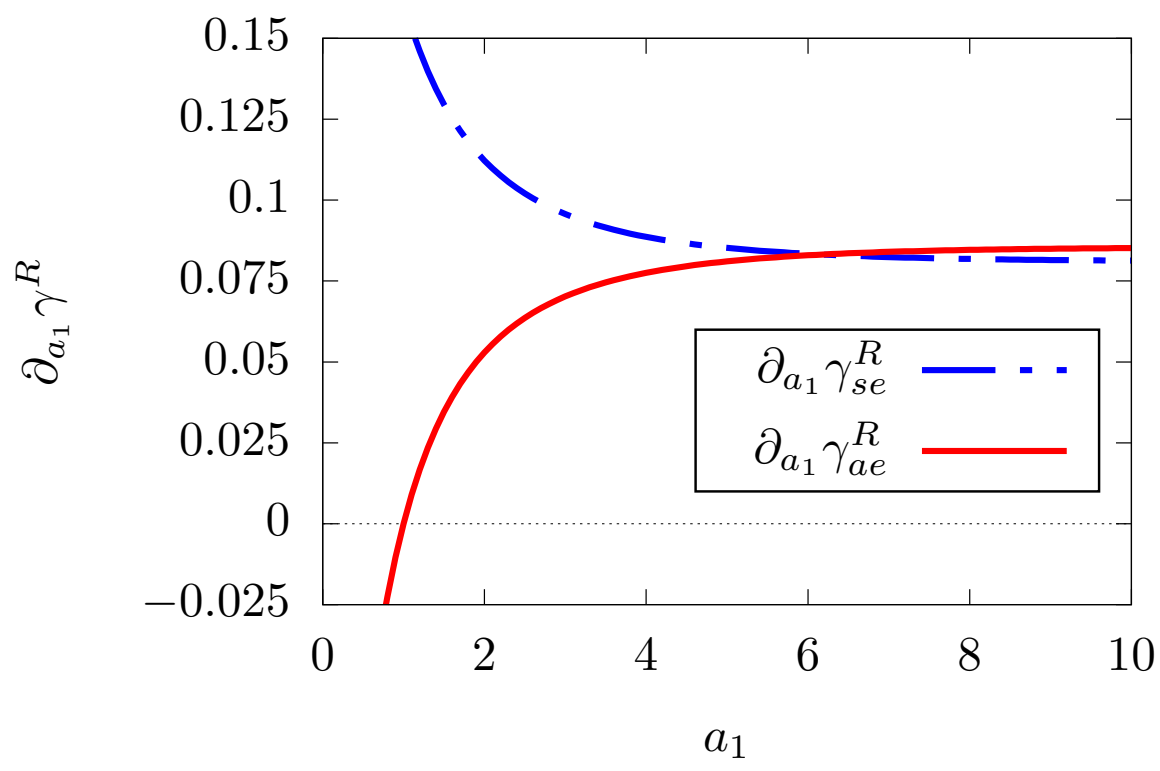

Figure 19. The quantity $\partial_{a_{1}} \gamma_{s e}^{R}$ is plotted with respect to varying $a_{1}$ for accelerated atoms in thermal background considering Rindler modes, depicted by dash-dotted blue line. The quantity $\partial_{a_{1}} \gamma_{a e}^{R}$ is plotted with respect to varying $a_{1}$ for accelerated atoms in thermal background considering Rindler modes in $(1+3)$ dimensions, depicted by solid red line.

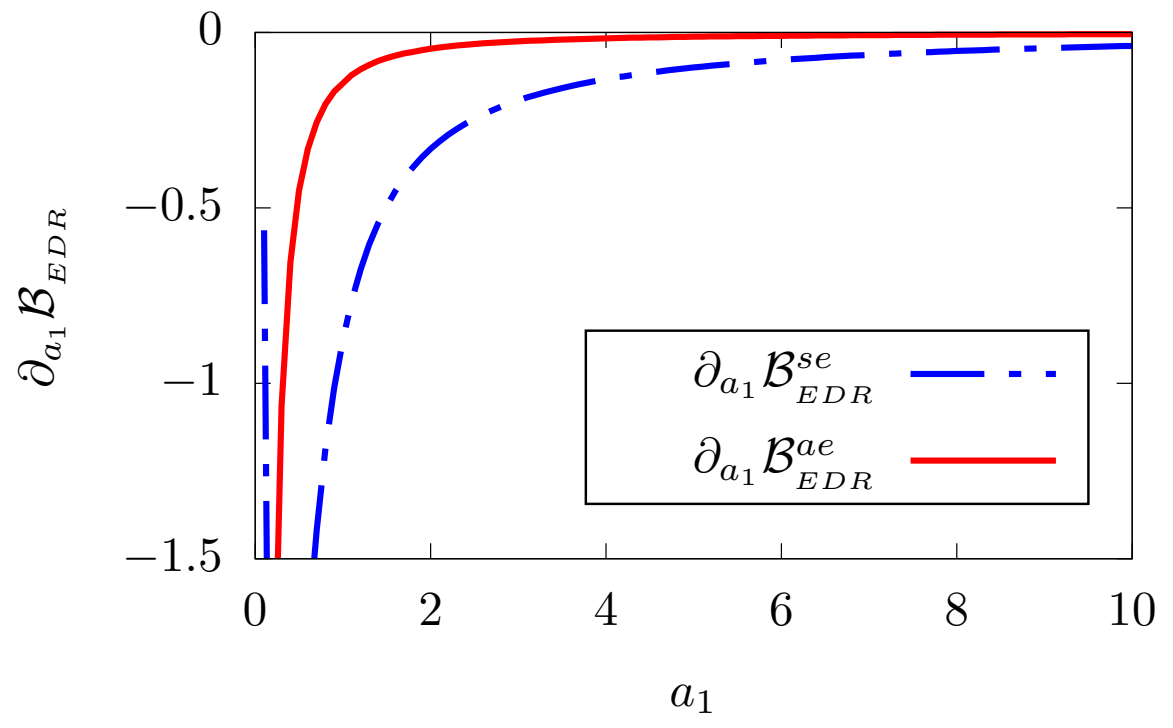

Figure 20. The quantity $\partial_{a_{1}} \mathcal{B}_{E D R}$ is plotted with respect to varying $a_{1}$ for accelerated atoms in thermal background considering Rindler modes in $(1+3)$ dimensions. For the transition from the symmetric state to the collective excited state the curve is given by the dash-dotted blue line. On the other hand, for the transition from the anti-symmetric state to the collective excited state the curve is given by the solid red line. The positivity of these curves are expected to provide the condition for strong anti-Unruh effect. 


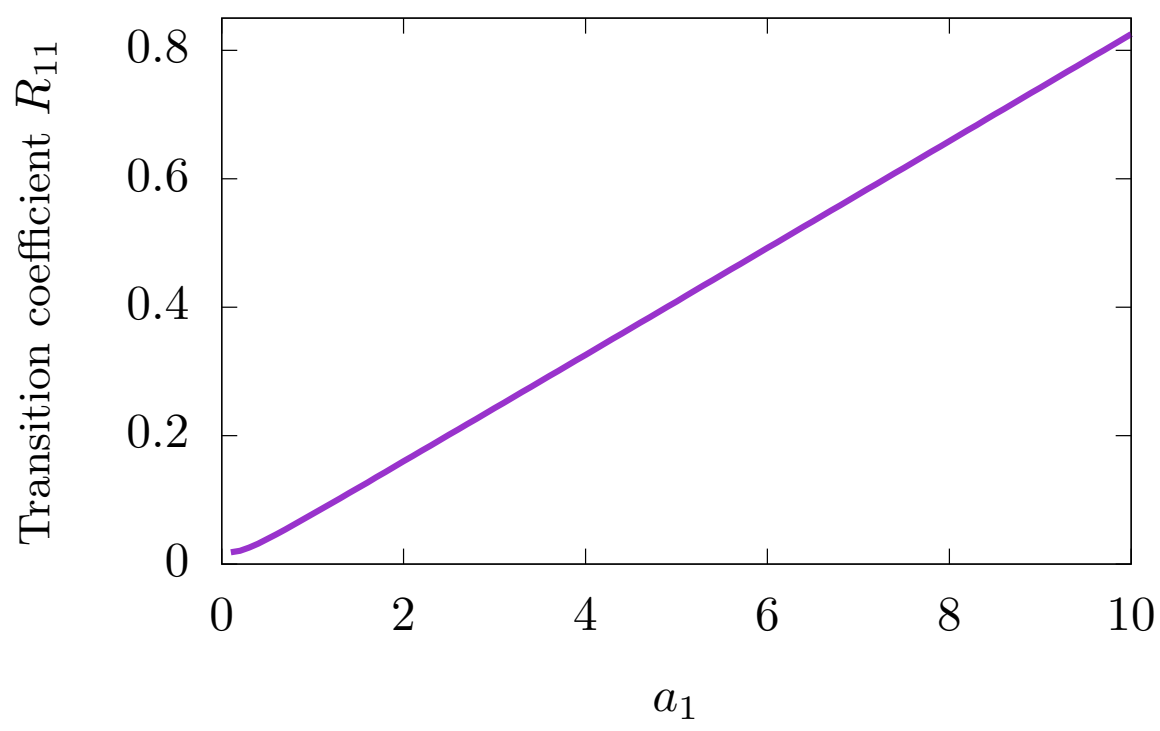

Figure 21. The response function $R_{11}(\Delta E)$, plotted with respect to varying $a_{1}$ for accelerated atoms in thermal background considering the Rindler modes in $(1+3)$ dimensions.

is no visible case of anti-Unruh effect for the selected set of values of the parameters, thus discarding the effects of the thermal bath in the occurrence of this anti-Unruh effect. It is in contrary to the case considering the Minkowski modes. Therefore, here also entanglement should be considered to be the significant contributor to the origin of the perceived antiUnruh phenomena, which can also be observed by plotting the $\partial_{a_{1}} \gamma_{a e}^{R}$ in the $\beta \rightarrow \infty$ limit. In figure 22 we have further checked the conditions for weak and strong anti-Unruh effect for $R_{11}(\Delta E)$, and found the absence of the same in the considered parameter range.

\subsection{Summarizing the outcomes regarding the anti-Unruh(-like) effect}

In this part we summarize the results that we have arrived on, while studying the antiUnruh effect considering two entangled atoms accelerated in a thermal bath. For the convenience of understanding the results will be tabulated below in a case by case manner. We shall first tabulate the results for the case with the Minkowski modes. Then we shall tabulate the results corresponding to the case with the Rindler modes.

In table 1 we have tabulated the results corresponding to the case with the Minkowski modes. On the other hand, in table 2 we have tabulated the results for the case with the Rindler modes.

In both of the cases the set of parameters signify the similar scenarios and are considered to be in the same range.

\section{Discussion}

In this work we have attempted to understand the radiative process of two entangled accelerated atoms interacting with a massless scalar field in a thermal bath. In particular, 


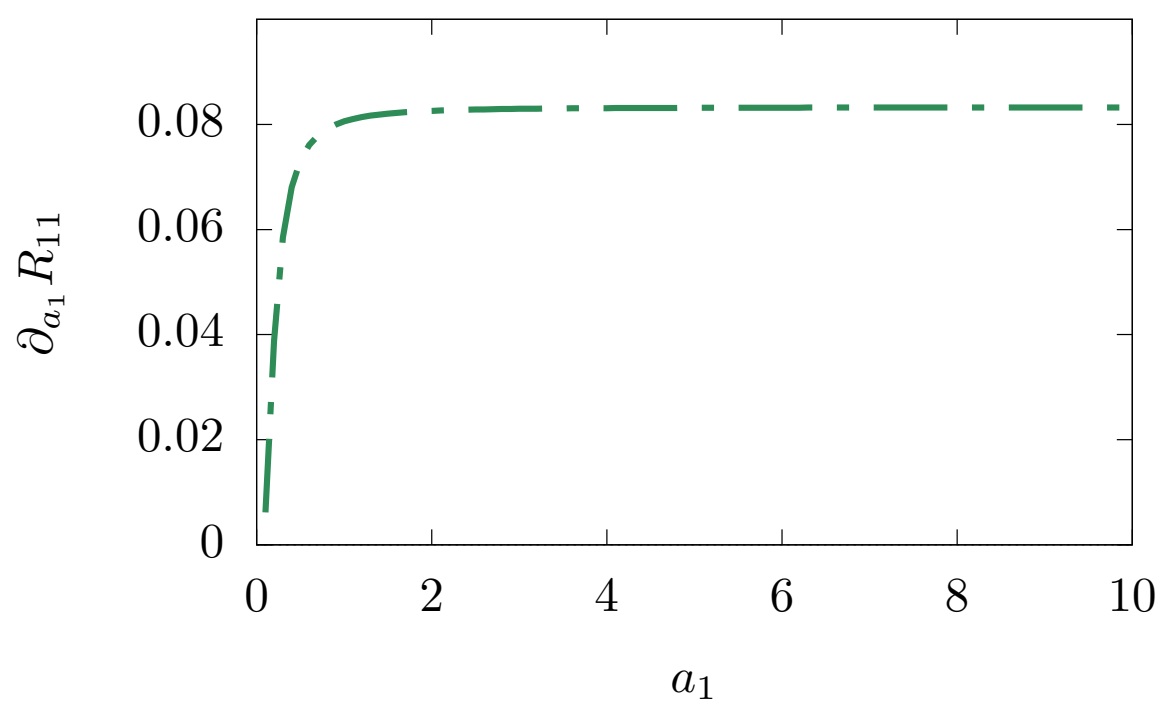

(a)

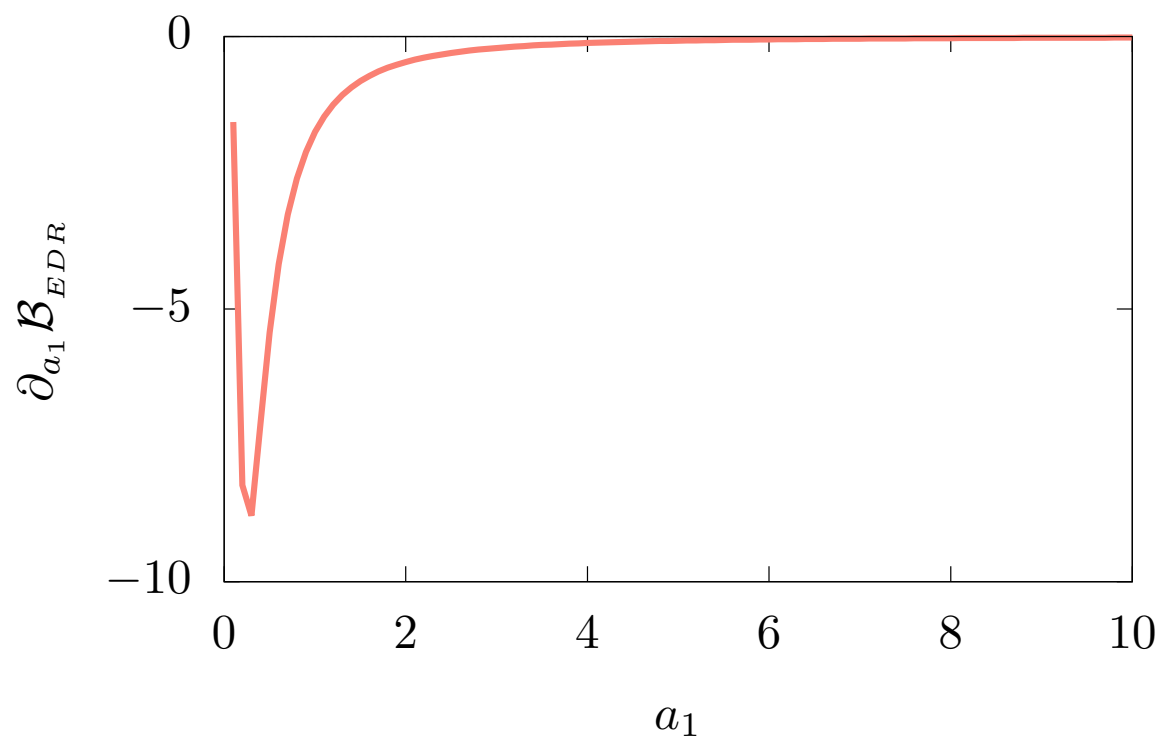

(b)

Figure 22. (a) The derivative of the response function $R_{11}(\Delta E)$, plotted with respect to varying acceleration $a_{1}$ for accelerated atoms in thermal background considering the Rindler modes in $(1+3)$ dimensions, denoted by dash-dotted green line. (b) In this figure the quantity $\partial_{a_{1}} \mathcal{B}_{E D R}$ corresponding to the response function is also plotted, which is denoted by the solid line. 


\begin{tabular}{|l|l|l|l|}
\hline \multirow{3}{*}{$(1+1)$ dimensions } & Transitions & Anti-Unruh-like effect & Nature \\
\cline { 2 - 4 } & $\gamma_{s e}$ & Yes & Entirely weak \\
\cline { 2 - 4 } & $\gamma_{a e}$ & Yes & Entirely weak \\
\cline { 2 - 4 } & $\mathcal{F}_{11}$ & Yes & Entirely weak \\
\hline \multirow{3}{*}{$(1+3)$ dimensions } & $\gamma_{s e}$ & Yes & Mostly weak, strong in some region \\
\cline { 2 - 4 } & $\gamma_{a e}$ & Yes & Mostly weak, strong in some region \\
\cline { 2 - 4 } & $\mathcal{F}_{11}$ & Yes & Entirely weak \\
\hline
\end{tabular}

Table 1. The case with the Minkowski modes.

\begin{tabular}{|l|l|l|l|}
\hline & Transitions & Anti-Unruh effect & Nature \\
\hline \multirow{5}{*}{$(1+1)$ dimensions } & $\gamma_{s e}^{R}$ & No & - \\
\cline { 2 - 4 } & $\gamma_{a e}^{R}$ & Yes & Both strong and weak \\
\cline { 2 - 4 } & $\mathcal{R}_{11}$ & No & - \\
\hline \multirow{3}{*}{$(1+3)$ dimensions } & $\gamma_{s e}^{R}$ & No & - \\
\cline { 2 - 4 } & $\gamma_{a e}^{R}$ & Yes & Entirely weak \\
\cline { 2 - 4 } & $\mathcal{R}_{11}$ & No & - \\
\hline
\end{tabular}

Table 2. The case with the Rindler modes.

the transitions form the symmetric and anti-symmetric entangled states to the collective excited or ground states are studied. It is to be noted that vacuum fluctuations effects acts as the cause for these transitions. In section 5 we have provided the estimations of the transition probabilities considering the Green's function, constructed from the Minkowski modes with a Rindler coordinate transformation, in both $(1+1)$ and $(1+3)$ dimensions. These transition probabilities correspond to certain frequencies of the field modes and they do not resemble the transition probabilities for unit time. In this case we observed that for both $(1+1)$ and $(1+3)$ dimensions there are visible cases of anti-Unruh-like effect in the transition probabilities. However, the $(1+1)$ dimensional results are qualitatively different from the $(1+3)$ dimensional one. For the transition from the symmetric state to the collective excited state we observed that in $(1+1)$ dimensions there is first antiUnruh-like effect and then Unruh-like effect, see from figure 2. However, for the same transition in $(1+3)$ dimensions there is only anti-Unruh-like effect in the same parameter range, see figure 3 . Therefore, there is a bit of difference between the $(1+1)$ and $(1+3)$ dimensional results. Another evidence of this mismatch is observed when the nature of the anti-Unruh-like effect is studied. In section 7 we observed that in $(1+1)$ dimensions the anti-Unruh-like effect is of purely weak nature in the considered parameter range. On the other hand, in $(1+3)$ dimensions in the same parameter range we observed that there are also some regions where strong condition is satisfied. The regions where the strong condition is satisfied are always contained inside the regions for the weak condition. We have further plotted the quantities $\mathcal{F}_{11}$ and observed the anti-Unruh-like effect here also, suggesting that in the Minkowski mode case entanglement do not play a significant role in the occurrence of the anti-Unruh-like effect, i.e., thermal background plays the major role. 
Furthermore, in section 6 we have considered the Green's functions in terms of the Rindler modes, obtained using the Unruh operators in the Unruh Vacuum, for the estimation of the transition probabilities. These transition probabilities are time translation invariant and a unit time prescription can be provided for them. Unlike the Minkowski mode case here the $(1+1)$ and $(1+3)$ dimensional results are in agreement with each other, see figure 4 and 5. For this case the occurrence of the anti-Unruh effect is confirmed only for the transition from the anti-symmetric state to the collective excited state, section 7 . However, the quantities $R_{11}$, which signify the contribution if a single detector were accelerated in the thermal bath, do not show any anti-Unruh effect in the selected parameter range. Thus suggesting that here entanglement has a significant role in the occurrence of the anti-Unruh effect.

Subsequently, the transition coefficients $\mathcal{F}_{11}$ considering the Minkowski modes are not symmetric under the interchange between the temperature of the thermal bath and the Unruh temperature, i.e., under $\beta \leftrightarrow 2 \pi / b$. However, for the case with the Rindler modes with Unruh operators $R_{11}$ is symmetric under the interchange $\beta \leftrightarrow 2 \pi / b$ (see appendix. A for qualitative difference in terms of time translation invariance, between the Green's functions considering the Minkowski and Rindler modes). Then the later case of Rindler modes with Unruh operators give a much more suitable representation for an accelerated observer, where the analogy with a thermal bath is concerned.

In summary through this work we have not only studied the radiative process of entangled atoms accelerated in a thermal bath but also provided understandings as to when an accelerated observer is invariably comparable to a static observer in thermal bath. These calculations and understandings motivate one to pursue other entanglement related studies $[21,22,26]$ such as entanglement dynamics, which includes rate of variation of the atomic energy, generation and decay of entangled states due to the contributions of vacuum fluctuations and radiation reaction, etc., for accelerated atoms in thermal bath.

\section{Acknowledgments}

S.B. would like to thank Indian Institute Technology Guwahati (IIT Guwahati) for supporting this work through a Post-Doctoral Fellowship.

\section{A Different Green's functions and the consequence of their consideration}

The fact that an accelerated observer in a Minkowski spacetime resembles an inertial observer in a thermal background is widely debated in literature. There are some studies $[46,50,51]$ in favour of this resemblance and there also articles pointing out some very crucial contradictions [54]. The discourse is still open and here we are going to provide some insightful results in this regard. Here we are going to point out the similarities and dissimilarities between observers in a thermal bath or in a non-inertial motion with uniform acceleration at the Green's function level. 


\section{A.1 Analogy between Green's functions of uniformly accelerated and static in thermal bath observers}

We mention that the momentum integral in eq. (3.4) can be explicitly carried out to provide a position space representation of the $(1+1)$ dimensional thermal Green's function as

$$
G_{\beta}^{+}\left(X_{2} ; X_{1}\right)=-\frac{1}{4 \pi}\left(\ln \left[1-e^{-\frac{2 \pi}{\beta}(\Delta T-\Delta X)}\right]+\ln \left[1-e^{-\frac{2 \pi}{\beta}(\Delta T+\Delta X)}\right]\right) .
$$

Similarly in $(1+3)$ dimensions also the thermal Green's function of eq. (3.5) can be explicitly evaluated in position space, see [54], as

$$
G_{\beta}^{+}\left(X_{2} ; X_{1}\right)=\frac{1}{8 \pi \beta|\Delta \mathbf{X}|}\left[\operatorname{coth}\left(\frac{\pi}{\beta}(\Delta T+|\Delta \mathbf{X}|)\right)-\operatorname{coth}\left(\frac{\pi}{\beta}(\Delta T-|\Delta \mathbf{X}|)\right)\right] .
$$

In the following discussions we are going to study these Green's functions in different limits and scenarios.

$(1+1)$ dimensions. One can take the expression of thermal Green's function in $(1+1)$ dimensions from eq. (A.1) and represent it in a more suitable manner for the subsequent analysis as

$$
G_{\beta}^{+}\left(X_{2} ; X_{1}\right)=-\frac{1}{4 \pi} \ln \left[\frac{\sinh \left(\frac{\pi}{\beta}(\Delta T-\Delta X)\right) \sinh \left(\frac{\pi}{\beta}(\Delta T+\Delta X)\right)}{\left(\frac{\pi}{\beta}\right)^{2}}\right] \frac{1}{2 \pi} \ln \left(\frac{2 \pi}{\beta} e^{-\frac{\pi \Delta T}{\beta}}\right) .
$$

From this expression of the Green's function one can arrive at the $(1+1)$ dimensional Minkowski Green's function as the limit $\beta \rightarrow \infty$ is taken. The corresponding expression is

$$
G_{M}^{+}\left(X_{2} ; X_{1}\right)=\frac{1}{4 \pi} \ln [(\Delta T-\Delta X)(\Delta T+\Delta X)] .
$$

However, it should be mentioned that this entire expression comes from the first term on the right hand side of eq. (A.3). The second term provides an infinite contribution, which is neglected out of convenience. Then neglecting this second term of eq. (A.3) one can observe that as one takes the limit $\Delta X \rightarrow 0$ the $(1+1)$ dimensional thermal Green's function reduces to

$$
G_{\beta}^{+}\left(X_{2} ; X_{1}\right)=-\frac{1}{2 \pi} \ln \left[\frac{\beta}{\pi} \sinh \left(\frac{\pi \Delta T}{\beta}\right)\right],
$$

which gives the Green's function corresponding to a static observer in thermal bath in $(1+1)$ dimensions. On the other hand, from the expression of $(1+1)$ dimensional Minkowski Green's function from eq. (A.4), and using the Rindler transformation of eq. (3.9) one can get the Green's function for an accelerated observer in $(1+1)$ dimensions as

$$
G_{M}^{+}\left(X_{2} ; X_{1}\right)=-\frac{1}{2 \pi} \ln \left[\frac{2}{b} \sinh \left(\frac{b \Delta \tau}{2}\right)\right] .
$$

It can be observed that the Green's functions from eq. (A.5) and eq. (A.6) are the same with $\Delta T$ and $\beta$ replaced by $\Delta \tau$ and $2 \pi / b$. 
$(\mathbf{1}+\mathbf{3})$ dimensions. We note that in eq. (A.2) as one takes $|\Delta \mathbf{X}| \rightarrow 0$, one shall get

$$
G_{\beta}^{+}\left(X_{2} ; X_{1}\right)=-\frac{1}{4 \beta^{2}} \frac{1}{\sinh ^{2}(\pi \Delta T / \beta)} .
$$

On the other hand, as one takes $1 / \beta \rightarrow 0$ in the expression of the Green's function from the same eq. (A.2), one can obtain the Minkowski Green's function

$$
G_{M}^{+}\left(X_{2} ; X_{1}\right)=\frac{1}{4 \pi^{2}} \frac{1}{\left(-\Delta T^{2}+|\Delta \mathbf{X}|^{2}\right)},
$$

which for an accelerated observer in terms of the Rindler proper time (3.9) can be expressed as

$$
G_{M}^{+}\left(X_{2} ; X_{1}\right)=-\frac{b^{2}}{16 \pi^{2}} \frac{1}{\sinh ^{2}(b \Delta \tau / 2)} .
$$

It should be noted that this Green's function and the thermal Green's function from eq. (A.7) have the exact same expression with $\Delta T$ and $\beta$ now replaced by $\Delta \tau$ and $2 \pi / b$. We have tabulated characteristics of these different Green's functions corresponding to static observer in thermal bath or uniformly accelerated observer in non-thermal background in table 3 .

\section{A.2 Analogy between Green's functions of accelerated atoms in thermal bath considering the Minkowski or Rindler modes, and atoms with double acceleration}

Accelerated observer in thermal bath. From eq. (3.10) with the coordinate transformation of eq. (3.11) one can find out the Green's function corresponding to an accelerated observer in a thermal bath in $(1+1)$ dimensions with respect to the Minkowski modes. Similarly, in $(1+3)$ dimensions the Green's function corresponding to an accelerated observer in thermal bath with respect to the Minkowski modes is given by the expression of eq. (3.12) with the coordinate transformation of eq. eq. (3.13). It should be noted that none of these Green's functions are time translation invariant with respect to the proper time of the accelerated observer.

On the other hand, the $(1+1)$ and $(1+3)$ dimensional Green's functions corresponding to accelerated observers in thermal bath with respect to the Rindler modes are given by eq. (4.9) and (4.16). It is to be noted that these Green's functions are time translation invariant. We further briefly discuss about the Green's function in a Rindler-Rindler frame.

Rindler-Rindler. In the first Rindler spacetime defined by the coordinate transformation of eq. (3.7) if one considers another analogous coordinate transformation, one can form the so called Rindler-Rindler spacetime. This coordinate transformation is

$$
\begin{aligned}
& \eta=\frac{e^{a^{\prime} x}}{a^{\prime}} \sinh a^{\prime} t \\
& \xi=\frac{e^{a^{\prime} x}}{a^{\prime}} \cosh a^{\prime} t,
\end{aligned}
$$




\begin{tabular}{|c|c|c|c|}
\hline & Green's function & $\begin{array}{l}\text { Time } \\
\text { translation } \\
\text { invariance }\end{array}$ & $\begin{array}{l}\text { Analogous } \\
\text { function }\end{array}$ \\
\hline \multirow[t]{6}{*}{$(1+1)$ dimensions } & \multirow[t]{2}{*}{ Thermal static } & \multirow[t]{2}{*}{ Yes } & $\begin{array}{l}\text { Uniformly accelerated } \\
\text { with Minkowski modes }\end{array}$ \\
\hline & & & $\begin{array}{l}\text { Uniformly accelerated } \\
\text { with Rindler modes }\end{array}$ \\
\hline & \multirow[t]{2}{*}{$\begin{array}{l}\text { Uniformly accelerated } \\
\text { with Minkowski modes }\end{array}$} & \multirow[t]{2}{*}{ Yes } & $\begin{array}{l}\text { Uniformly accelerated } \\
\text { with Rindler modes }\end{array}$ \\
\hline & & & Thermal static \\
\hline & \multirow[t]{2}{*}{$\begin{array}{l}\text { Uniformly accelerated } \\
\text { with Rindler modes }\end{array}$} & \multirow[t]{2}{*}{ Yes } & $\begin{array}{l}\text { Uniformly accelerated } \\
\text { with Minkowski modes }\end{array}$ \\
\hline & & & Thermal static \\
\hline \multirow[t]{6}{*}{$(1+3)$ dimensions } & \multirow[t]{2}{*}{ Thermal static } & \multirow[t]{2}{*}{ Yes } & $\begin{array}{l}\text { Uniformly accelerated } \\
\text { with Minkowski modes }\end{array}$ \\
\hline & & & $\begin{array}{l}\text { Uniformly accelerated } \\
\text { with Rindler modes }\end{array}$ \\
\hline & \multirow[t]{2}{*}{$\begin{array}{l}\text { Uniformly accelerated } \\
\text { with Minkowski modes }\end{array}$} & \multirow[t]{2}{*}{ Yes } & $\begin{array}{l}\text { Uniformly accelerated } \\
\text { with Rindler modes }\end{array}$ \\
\hline & & & Thermal static \\
\hline & \multirow[t]{2}{*}{$\begin{array}{l}\text { Uniformly accelerated } \\
\text { with Rindler modes }\end{array}$} & \multirow[t]{2}{*}{ Yes } & $\begin{array}{l}\text { Uniformly accelerated } \\
\text { with Minkowski modes }\end{array}$ \\
\hline & & & Thermal static \\
\hline
\end{tabular}

Table 3. Characteristics of different Green's functions (observers with uniform acceleration or static in thermal bath).

which enables one to express the line element as

$$
d s^{2}=e^{2 a^{\prime} x} \exp \left\{\frac{2 a}{a^{\prime}} e^{a^{\prime} x} \cosh a^{\prime} t\right\}\left[-d t^{2}+d x^{2}\right] .
$$

It should be mentioned that here $a$ and $a^{\prime}$ denote the acceleration parameters corresponding to the first and the second Rindler transformations. The relation between the Minkowski coordinates $(T, X)$ and thees Rindler-Rindler coordinates $(t, x)$ is

$$
\begin{aligned}
& T=\frac{\tilde{X}}{a} \sinh \tilde{T} \frac{e^{-a / a^{\prime}}}{2 a}\left\{\exp \left[\frac{a}{a^{\prime}} e^{a^{\prime}(x+t)}\right]-\exp \left[\frac{a}{a^{\prime}} e^{a^{\prime}(x-t)}\right]\right\} \\
& X=\frac{\tilde{X}}{a} \cosh \tilde{T}=\frac{e^{-a / a^{\prime}}}{2 a}\left\{\exp \left[\frac{a}{a^{\prime}} e^{a^{\prime}(x+t)}\right]+\exp \left[\frac{a}{a^{\prime}} e^{a^{\prime}(x-t)}\right]\right\} .
\end{aligned}
$$

Here $\tilde{X}=\exp \left[\frac{a}{a^{\prime}} e^{a^{\prime} x} \cosh a^{\prime} t\right]$ and $\tilde{T}=\frac{a}{a^{\prime}} e^{a^{\prime} x} \sinh a^{\prime} t$. Then we have the expression of

$$
\begin{aligned}
T+X & =\frac{e^{-a / a^{\prime}}}{a} \exp \left[\frac{a}{a^{\prime}} e^{a^{\prime}(x+t)}\right] \\
T-X & =-\frac{e^{-a / a^{\prime}}}{a} \exp \left[\frac{a}{a^{\prime}} e^{a^{\prime}(x-t)}\right],
\end{aligned}
$$




\begin{tabular}{|c|c|c|c|}
\hline & Green's function & $\begin{array}{l}\text { Time translation } \\
\text { invariance }\end{array}$ & $\begin{array}{l}\text { Analogous Green's } \\
\text { function }\end{array}$ \\
\hline \multirow[t]{3}{*}{$(1+1)$ dimensions } & $\begin{array}{l}\text { Accelerated in } \\
\text { thermal bath with } \\
\text { Minkowski modes }\end{array}$ & No & - \\
\hline & $\begin{array}{l}\text { Accelerated in } \\
\text { thermal bath with } \\
\text { Rindler modes }\end{array}$ & Yes & - \\
\hline & Double acceleration & - & - \\
\hline \multirow[t]{3}{*}{$(1+3)$ dimensions } & $\begin{array}{l}\text { Accelerated in } \\
\text { thermal bath with } \\
\text { Minkowski modes }\end{array}$ & No & - \\
\hline & $\begin{array}{l}\text { Accelerated in } \\
\text { thermal bath with } \\
\text { Rindler modes }\end{array}$ & Yes & - \\
\hline & Double acceleration & - & - \\
\hline
\end{tabular}

Table 4. Characteristics of different Green's functions (observers with uniform acceleration in thermal bath or with double acceleration).

which can be used to obtain the $(1+1)$ and $(1+3)$ dimensional Green's functions from eq. (A.4) and (A.8) corresponding to an observer in a Rindler-Rindler frame with respect to the Minkowski modes. It should be mentioned that in a Rindler-Rindler frame the exact expression of the proper time is not yet known up to our knowledge. Then it is not readily possible to comment about the time translational invariance for this Green's function. However, structure wise it can be observed that it is different than the thermal-Rindler case. We have tabulated characteristics of different Green's functions, corresponding to accelerated observers in thermal bath or observer in Rindler-Rindler frame, and the analogy between them in table 4 .

\section{B Green's function of accelerated observer considering Rindler modes}

To obtain the Green's function of an accelerated observer considering the Rindler modes we first take the definition of the Green's function

$$
G_{R}^{+}\left(X_{2}, X_{1}\right)=\left\langle 0_{M}\left|\Phi^{R}\left(X_{2}\right) \Phi^{R}\left(X_{1}\right)\right| 0_{M}\right\rangle .
$$

In this expression we put the expression of the scalar field from eq. (4.7), which denotes the scalar field decomposition in the right Rindler wedge. We also assume that for both of the spacetime points acceleration is the same $a$. Then the above Green's function (B.1) 
becomes

$$
\begin{aligned}
G_{R}^{+}= & \left\langle 0_{M}\right| \sum_{k, k^{\prime}=-\infty}^{\infty} \frac{1}{2 \sqrt{\sinh \frac{\pi \omega_{k}}{a} \sinh \frac{\pi \omega_{k^{\prime}}}{a}}} \\
& \times\left[\left(d_{k}^{1} e^{\frac{\pi \omega_{k}}{2 a} R} u_{k}+d_{k}^{2} e^{-\frac{\pi \omega_{k}}{2 a}} R u_{-k}^{*}\right)\left(d_{k^{\prime}}^{1^{\dagger}} e^{\frac{\pi \omega_{k^{\prime}}}{2 a}} R u_{k^{\prime}}^{*}+d_{k^{\prime}}^{2^{\dagger}} e^{-\frac{\pi \omega_{k^{\prime}}}{2 a} R} u_{-k^{\prime}}\right)\right]\left|0_{M}\right\rangle \\
= & \sum_{k=-\infty}^{\infty} \frac{1}{2 \sinh \frac{\pi \omega_{k}}{a}}\left[e^{\frac{\pi \omega_{k}}{a}} R u_{k} R_{k^{*}}+e^{-\frac{\pi \omega_{k}}{a}} R u_{-k}^{*} R_{-k}\right],
\end{aligned}
$$

where, we have used the commutation relation $\left[d_{k}^{j}, d_{k^{\prime}}^{j^{\dagger}}\right]=\delta_{k, k^{\prime}}$. Now by putting the expression of the modes ${ }^{R} u_{k}$ from eq. (4.1) one can obtain the Green's function to be

$$
G_{R}^{+}=\sum_{k=-\infty}^{\infty} \frac{1}{4 \pi \omega_{k}}\left[\frac{e^{i k \Delta \xi-i \omega_{k} \Delta \eta}}{1-e^{\frac{-2 \pi \omega_{k}}{a}}}+\frac{e^{i k \Delta \xi+i \omega_{k} \Delta \eta}}{e^{\frac{2 \pi \omega_{k}}{a}}-1}\right],
$$

which, in the continuum momentum limit yields the desired expression of the Green's function for an accelerated observer in terms of the Rindler modes as given in eq. (4.8). Note that this evaluation is done for $(1+1)$ dimensions. A similar evaluation can be done in the $(1+3)$ dimensions also considering the scalar field expansion in RRW from eq. (4.14) and putting it in eq. (B.1) and then using the expressions of the mode from eq. (4.10) to evaluate the Green's function corresponding to an accelerated observer in terms of the Rindler modes, the expression of which is given in eq. (4.15).

Next we construct the Green's function in the Minkowski vacuum considering the field decomposition given by eq. (4.4), i.e., in terms of the Unruh modes and Unruh operators in $(1+1)$ dimensions. This Green's function is obtained as

$$
G_{U}^{+}\left(X_{2}, X_{1}\right)=\left\langle 0_{M}\left|\Phi\left(X_{2}\right) \Phi\left(X_{1}\right)\right| 0_{M}\right\rangle=G_{R}^{+}\left(X_{2}, X_{1}\right)+G_{L}^{+}\left(X_{2}, X_{1}\right)+G_{R L}^{+}\left(X_{2}, X_{1}\right),
$$

where the expressions $G_{R}^{+}\left(X_{2}, X_{1}\right)$ and $G_{L}^{+}\left(X_{2}, X_{1}\right)$ correspond to accelerated observers in the right and in left Rindler wedges respectively, and $G_{R L}^{+}\left(X_{2}, X_{1}\right)$ denotes the cross term. The expression of $G_{R}^{+}\left(X_{2}, X_{1}\right)$ is already given in eq. (B.2) and the other two quantities are given by

$$
\begin{aligned}
G_{L}^{+}\left(X_{2}, X_{1}\right) & =\sum_{k=-\infty}^{\infty} \frac{1}{2 \sinh \frac{\pi \omega_{k}}{a}}\left[e^{\frac{\pi \omega_{k}}{a}} L_{u_{k}}{ }^{L} u_{k}^{*}+e^{-\frac{\pi \omega_{k}}{a} L} u_{-k}^{*}{ }^{L} u_{-k}\right], \\
G_{R L}^{+}\left(X_{2}, X_{1}\right) & =\sum_{k=-\infty}^{\infty} \frac{1}{2 \sinh \frac{\pi \omega_{k}}{a}}\left[{ }^{R} u_{k}^{L} u_{-k}+{ }^{L} u_{-k}^{*}{ }^{R} u_{k}^{*}+{ }^{L} u_{k}^{R} u_{-k}+{ }^{R} u_{-k}^{*}{ }^{L} u_{k}^{*}\right] .
\end{aligned}
$$

One can use the explicit expressions of the Rindler field modes ${ }^{R} u_{k}$ and ${ }^{L} u_{k}$ from eq. (4.1) and obtain the expression of $G_{R}^{+}\left(X_{2}, X_{1}\right)$ same as given in eq. (B.3) and other expressions of $G_{L}^{+}\left(X_{2}, X_{1}\right)$ and $G_{R L}^{+}\left(X_{2}, X_{1}\right)$ in a similar manner. A $(1+3)$ dimensional representation of this Green's function can be obtained in a similar manner. 


\section{Green's function of accelerated observer in thermal bath considering Rindler modes}

Considering Gibbs ensemble average definition from eq. (3.1), and Rindler mode decomposition of the scalar field from eq. (4.7) we obtain the Green's function of an accelerated observer in a thermal bath as

$$
\begin{aligned}
G_{\beta_{R}}^{+}\left(X_{j, 2}, X_{l, 1}\right)= & \sum_{k, k^{\prime}=-\infty}^{\infty} \frac{1}{2 \sqrt{\sinh \frac{\pi \omega_{k}}{a_{j}} \sinh \frac{\pi \omega_{k^{\prime}}}{a_{l}}}} \\
& \times\left\langle\left[ d_{k}^{1} d_{k^{\prime}}^{1^{\dagger}} e^{\frac{\pi}{2}\left(\frac{\omega_{k}}{a_{j}}+\frac{\omega_{k^{\prime}}}{a_{l}}\right)} R u_{k}^{j} R u_{k^{\prime}}^{l *}+d_{k}^{1^{\dagger}} d_{k^{\prime}}^{1} e^{\frac{\pi}{2}\left(\frac{\omega_{k}}{a_{j}}+\frac{\omega_{k^{\prime}}}{a_{l}}\right) R} u_{k}^{j * R} u_{k^{\prime}}^{l}\right.\right. \\
& \left.\left.+d_{k}^{2} d_{k^{\prime}}^{2^{\dagger}} e^{-\frac{\pi}{2}\left(\frac{\omega_{k}}{a_{j}}+\frac{\omega_{k^{\prime}}}{a_{l}}\right)} R u_{-k}^{j *} R u_{-k^{\prime}}^{l}+d_{k}^{2^{\dagger}} d_{k^{\prime}}^{2} e^{-\frac{\pi}{2}\left(\frac{\omega_{k}}{a_{j}}+\frac{\omega_{k^{\prime}}}{a_{l}}\right)} R u_{-k}^{j}{ }^{R} u_{-k^{\prime}}^{l *}\right]\right\rangle_{\beta}
\end{aligned}
$$

Here $\langle\hat{O}\rangle_{\beta}$ denotes the Gibbs ensemble average and the superscript $j$ (or $l$ ) denotes the $j^{\text {th }}$ (or $l^{\text {th }}$ detector) which corresponds to the second(or first) spacetime point. We mention that the observer is considered to be confined in the right Rindler wedge. Furthermore, we consider the Hamiltonian corresponding to the $k^{\text {th }}$ excitation to be $H_{k}=\left(d_{k}^{1^{\dagger}} d_{k}^{1}+\right.$ $\left.d_{k}^{2^{\dagger}} d_{k}^{2}\right) \omega_{k}$. Then the Gibbs ensemble average of the operators $\left\langle d_{k}^{j} d_{k^{\prime}}^{j^{\dagger}}\right\rangle_{\beta}=\delta_{k, k^{\prime}} /\left(1-e^{-\beta \omega_{k}}\right)$ and $\left\langle d_{k}^{j^{\dagger}} d_{k^{\prime}}^{j}\right\rangle_{\beta}=\delta_{k, k^{\prime}} /\left(e^{\beta \omega_{k}}-1\right)$. These results can be used along with the expression of the modes ${ }^{R} u_{k}$ from eq. (4.1) to obtain the desired expression of the Green's function from eq. (4.9). Here also this evaluation is provided for $(1+1)$ dimensions. One can evaluate the Green's function corresponding to accelerated detectors in thermal bath for $(1+3)$ dimensions considering the Rindler modes as given in eq. (4.16) in a similar manner. However, in the later case the scalar field expansion in RRW and the expression of the Rindler modes are taken from eq. (4.7) and (4.10).

Next we consider the Gibbs ensemble average definition from eq. (3.1) to obtain the Green's function in a thermal bath taking the field decomposition given by eq. (4.4), i.e., in terms of the Unruh modes and Unruh operators in $(1+1)$ dimensions. This Green's function looks like

$$
G_{\beta_{U}}^{+}\left(X_{j, 2}, X_{l, 1}\right)=G_{\beta_{R}}^{+}\left(X_{j, 2}, X_{l, 1}\right)+G_{\beta_{L}}^{+}\left(X_{j, 2}, X_{l, 1}\right)+G_{\beta_{R L}}^{+}\left(X_{j, 2}, X_{l, 1}\right)
$$

Here the expressions $G_{\beta_{R}}^{+}\left(X_{j, 2}, X_{l, 1}\right)$ and $G_{\beta_{L}}^{+}\left(X_{j, 2}, X_{l, 1}\right)$ correspond to accelerated observers in thermal bath in the right and in left Rindler wedges respectively, and $G_{\beta_{R L}}^{+}\left(X_{j, 2}, X_{l, 1}\right)$ denotes the cross term. The expression of $G_{\beta_{R}}^{+}\left(X_{j, 2}, X_{l, 1}\right)$ can be obtained 
from eq. (C.1) and the other two quantities are given by

$$
\begin{aligned}
& G_{\beta_{L}}^{+}\left(X_{j, 2}, X_{l, 1}\right)=\sum_{k=-\infty}^{\infty} \frac{1}{2 \sqrt{\sinh \frac{\pi \omega_{k}}{a_{j}} \sinh \frac{\pi \omega_{k}}{a_{l}}}} \\
& \times\left[\frac{1}{1-e^{-\beta \omega_{k}}}\left\{e^{\frac{\pi \omega_{k}}{2}\left(\frac{1}{a_{j}}+\frac{1}{a_{l}}\right)} L u_{k}^{j} L u_{k}^{l *}+e^{-\frac{\pi \omega_{k}}{2}\left(\frac{1}{a_{j}}+\frac{1}{a_{l}}\right)} L_{u_{-k}^{j *}} L_{u_{-k}^{l}}^{l}\right\}\right. \\
& \left.+\frac{1}{e^{\beta \omega_{k}}-1}\left\{e^{\frac{\pi \omega_{k}}{2}\left(\frac{1}{a_{j}}+\frac{1}{a_{l}}\right)} L_{u_{k}^{j} * L} u_{k}^{l}+e^{-\frac{\pi \omega_{k}}{2}\left(\frac{1}{a_{j}}+\frac{1}{a_{l}}\right)} L_{u_{-k}^{j}} L_{u_{-k} l^{*}}\right\}\right], \\
& G_{\beta_{R L}}^{+}\left(X_{j, 2}, X_{l, 1}\right)=\sum_{k=-\infty}^{\infty} \frac{1}{2 \sqrt{\sinh \frac{\pi \omega_{k}}{a_{j}} \sinh \frac{\pi \omega_{k}}{a_{l}}}} \\
& \times\left[\frac { 1 } { 1 - e ^ { - \beta \omega _ { k } } } \left\{e^{\frac{\pi \omega_{k}}{2}\left(\frac{1}{a_{j}}-\frac{1}{a_{l}}\right)} R u_{k}^{j}{ }^{L} u_{-k}^{l}+e^{-\frac{\pi \omega_{k}}{2}\left(\frac{1}{a_{j}}-\frac{1}{a_{l}}\right)}{ } u_{-k}^{j * R} u_{k}^{l} *\right.\right. \\
& \left.+e^{\frac{\pi \omega_{k}}{2}\left(\frac{1}{a_{j}}-\frac{1}{a_{l}}\right)} L u_{k}^{j} R u_{-k}^{l}+e^{-\frac{\pi \omega_{k}}{2}\left(\frac{1}{a_{j}}-\frac{1}{a_{l}}\right)} R u_{-k}^{j * L} u_{k}^{l *}\right\} \\
& +\frac{1}{e^{\beta \omega_{k}}-1}\left\{e^{\frac{\pi \omega_{k}}{2}\left(\frac{1}{a_{j}}-\frac{1}{a_{l}}\right)} R u_{k}^{j * L} u_{-k}^{l *}+e^{-\frac{\pi \omega_{k}}{2}\left(\frac{1}{a_{j}}-\frac{1}{a_{l}}\right)} L_{u_{-k}^{j}} R u_{k}^{l}\right. \\
& \left.\left.+e^{\frac{\pi \omega_{k}}{2}\left(\frac{1}{a_{j}}-\frac{1}{a_{l}}\right) L} u_{k}^{j * R} u_{-k}^{l *}+e^{-\frac{\pi \omega_{k}}{2}\left(\frac{1}{a_{j}}-\frac{1}{a_{l}}\right)} R u_{-k}^{j} L u_{k}^{l}\right\}\right] .
\end{aligned}
$$

One can use the explicit expressions of the Rindler field modes ${ }^{R} u_{k}$ and ${ }^{L} u_{k}$ from eq. (4.1) and further express the quantities $G_{\beta_{R}}^{+}\left(X_{2}, X_{1}\right), G_{\beta_{L}}^{+}\left(X_{2}, X_{1}\right)$, and $G_{\beta_{R L}}^{+}\left(X_{2}, X_{1}\right)$ in terms of Rindler coordinates. Here also a $(1+3)$ dimensional representation of this Green's function can be provided in a similar manner.

\section{Relation between the detector proper times}

We take the Minkowski to Rindler coordinate transformation from eq. (3.7) to express the two accelerated observers. In particular the coordinate transformation corresponding to our first accelerated observer is

$$
\begin{aligned}
T_{1} & =\frac{e^{a_{1} \xi_{1}}}{a_{1}} \sinh a_{1} \eta_{1} \\
X_{1} & =\frac{e^{a_{1} \xi_{1}}}{a_{1}} \cosh a_{1} \eta_{1},
\end{aligned}
$$

and a similar coordinate transformation corresponding to the second accelerated observer is

$$
\begin{aligned}
T_{2} & =\frac{e^{a_{2} \xi_{2}}}{a_{2}} \sinh a_{2} \eta_{2} \\
X_{2} & =\frac{e^{a_{2} \xi}}{a_{2}} \cosh a_{2} \eta_{2} .
\end{aligned}
$$




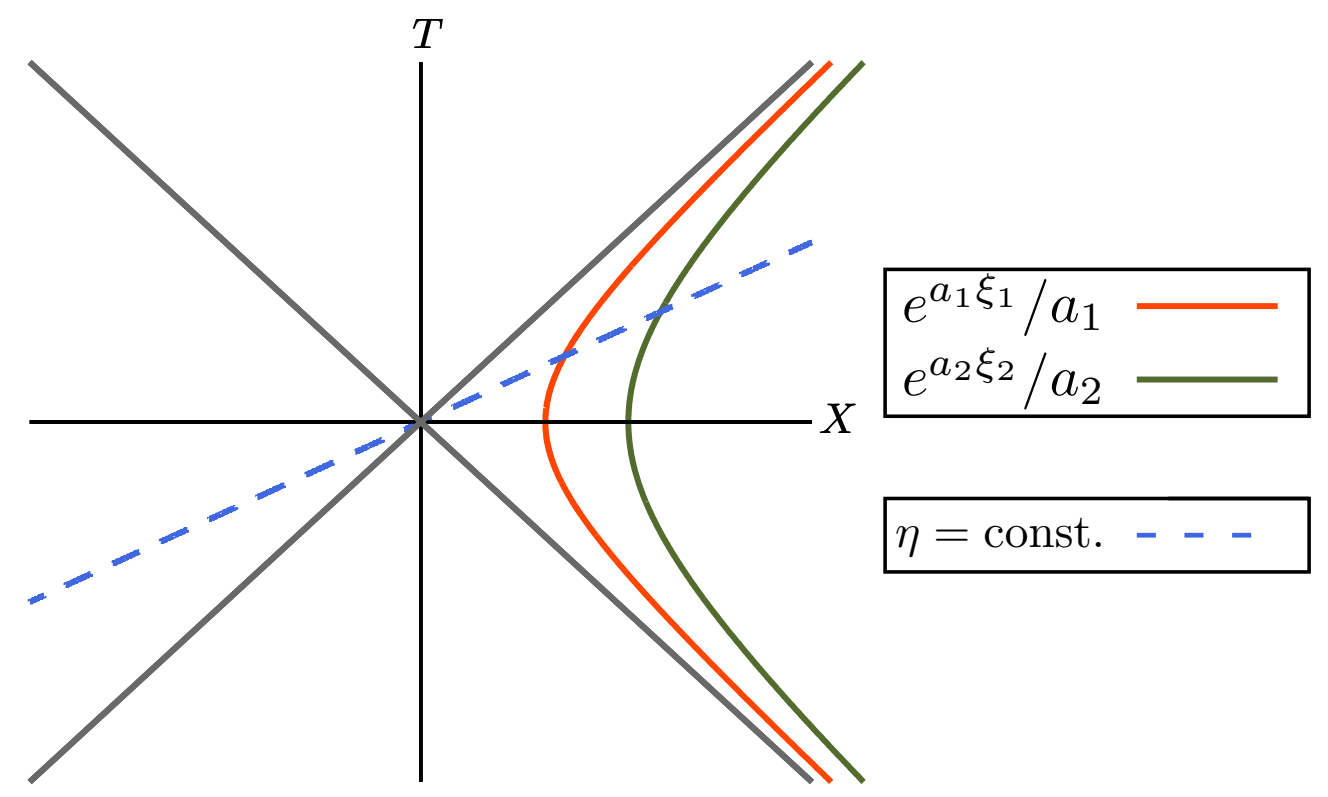

Figure 23. Here the trajectories of two observers with two different proper accelerations are depicted in the right Rindler wedge.

From these coordinate transformations one can observe that $\eta_{j}=\left(1 / a_{j}\right) \tanh ^{-1}\left(T_{j} / X_{j}\right)$ and $e^{a_{j} \xi_{j}} / a_{j}=\left(X_{j}^{2}-T_{j}^{2}\right)^{-1 / 2}$. It means constant Rindler times signify straight lines in the Minkowski $T-X$ plane, see figure 23. On the other hand observers with constant proper accelerations $b_{j}=a_{j} e^{-a_{j} \xi_{j}}$ follow the hyperbolic trajectories, also depicted in the figure 23 . Now the scenario of constant proper acceleration can be achieved in a few different ways. One can take two accelerated observers with the same Rindler parameter $a_{1}=a_{2}$ and keep them in two different Rindler spatial points $\xi_{1} \neq \xi_{2}$. On the other, hand one can also take the $a_{1} \neq a_{2}$ from the beginning with the consideration of $\xi_{1}=\xi_{2}$. In both of the cases the observers have different proper acceleration, i.e., they signify two different hyperbolic trajectories in the Minkowski $T-X$ plane. Interestingly in both of the cases these trajectories can be cut by a single $\eta=$ const. line. It is noticed that if one considers both of the observers to be described by equal Rindler time $\eta$ then a relation between the proper times of the two different observers can be obtained.

Open Access. This article is distributed under the terms of the Creative Commons Attribution License (CC-BY 4.0), which permits any use, distribution and reproduction in any medium, provided the original author(s) and source are credited.

\section{References}

[1] B.T. Kirby and J.D. Franson, Nonlocal interferometry using macroscopic coherent states and weak nonlinearities, Phys. Rev. A 87 (2013) 053822.

[2] B. Hensen et al., Loophole-free Bell inequality violation using electron spins separated by 1.3 kilometres, Nature 526 (2015) 682 [arXiv:1508.05949] [INSPIRE]. 
[3] W. Tittel, J. Brendel, H. Zbinden and N. Gisin, Violation of Bell inequalities by photons more than $10 \mathrm{~km}$ apart, Phys. Rev. Lett. 81 (1998) 3563 [quant-ph/9806043] [INSPIRE].

[4] D. Salart, A. Baas, C. Branciard, N. Gisin and H. Zbinden, Testing the speed of 'spooky action at a distance', Nature 454 (2008) 861.

[5] I. Fuentes-Schuller and R.B. Mann, Alice falls into a black hole: entanglement in non-inertial frames, Phys. Rev. Lett. 95 (2005) 120404 [quant-ph/0410172] [INSPIRE].

[6] B. Reznik, Entanglement from the vacuum, Found. Phys. 33 (2003) 167 [quant-ph/0212044] [INSPIRE].

[7] S.-Y. Lin and B.L. Hu, Entanglement creation between two causally disconnected objects, Phys. Rev. D 81 (2010) 045019 [arXiv:0910.5858] [InSPIRE].

[8] J.L. Ball, I. Fuentes-Schuller and F.P. Schuller, Entanglement in an expanding spacetime, Phys. Lett. A 359 (2006) 550 [quant-ph/0506113] [INSPIRE].

[9] M. Cliche and A. Kempf, The relativistic quantum channel of communication through field quanta, Phys. Rev. A 81 (2010) 012330 [arXiv:0908.3144] [INSPIRE].

[10] E. Martin-Martinez and N.C. Menicucci, Cosmological quantum entanglement, Class. Quant. Grav. 29 (2012) 224003 [arXiv: 1204.4918] [INSPIRE].

[11] G. Salton, R.B. Mann and N.C. Menicucci, Acceleration-assisted entanglement harvesting and rangefinding, New J. Phys. 17 (2015) 035001 [arXiv:1408.1395] [INSPIRE].

[12] E. Martin-Martinez, A.R.H. Smith and D.R. Terno, Spacetime structure and vacuum entanglement, Phys. Rev. D 93 (2016) 044001 [arXiv:1507.02688] [INSPIRE].

[13] H. Cai and Z. Ren, Transition processes of a static multilevel atom in the cosmic string spacetime with a conducting plane boundary, Sci. Rep. 8 (2018) 11802 [INSPIRE].

[14] G. Menezes, Entanglement dynamics in a Kerr spacetime, Phys. Rev. D 97 (2018) 085021 [arXiv: 1712.07151] [INSPIRE].

[15] G. Menezes, N.F. Svaiter and C.A.D. Zarro, Entanglement dynamics in random media, Phys. Rev. A 96 (2017) 062119 [arXiv:1709.08702] [InSPIRE].

[16] W. Zhou and H. Yu, Resonance interatomic energy in a Schwarzschild spacetime, Phys. Rev. D 96 (2017) 045018 [INSPIRE].

[17] L.J. Henderson, R.A. Hennigar, R.B. Mann, A.R.H. Smith and J. Zhang, Harvesting entanglement from the black hole vacuum, Class. Quant. Grav. 35 (2018) $21 \mathrm{LT02}$ [arXiv: 1712.10018] [INSPIRE].

[18] L.J. Henderson and N.C. Menicucci, Bandlimited entanglement harvesting, Phys. Rev. D 102 (2020) 125026 [arXiv:2005.05330] [INSPIRE].

[19] N. Stritzelberger, L.J. Henderson, V. Baccetti, N.C. Menicucci and A. Kempf, Entanglement harvesting with coherently delocalized matter, Phys. Rev. D 103 (2021) 016007 [arXiv: 2006.11291] [INSPIRE].

[20] C.D. Rodríguez-Camargo, N.F. Svaiter and G. Menezes, Finite-time response function of uniformly accelerated entangled atoms, Annals Phys. 396 (2018) 266 [arXiv: 1608.03365] [INSPIRE].

[21] G. Menezes and N.F. Svaiter, Radiative processes of uniformly accelerated entangled atoms, Phys. Rev. A 93 (2016) 052117 [arXiv:1512.02886] [INSPIRE]. 
[22] J. Hu and H. Yu, Entanglement dynamics for uniformly accelerated two-level atoms, Phys. Rev. A 91 (2015) 012327 [arXiv: 1501.03321] [INSPIRE].

[23] L. Rizzuto et al., Nonthermal effects of acceleration in the resonance interaction between two uniformly accelerated atoms, Phys. Rev. A 94 (2016) 012121 [arXiv:1601.04502] [INSPIRE].

[24] E. Arias, J.G. Dueñas, G. Menezes and N.F. Svaiter, Boundary effects on radiative processes of two entangled atoms, JHEP 07 (2016) 147 [arXiv:1510.00047] [INSPIRE].

[25] G. Picanço, N.F. Svaiter and C.A.D. Zarro, Radiative processes of entangled detectors in rotating frames, JHEP 08 (2020) 025 [arXiv: 2002.06085] [INSPIRE].

[26] W. Zhou and H. Yu, Radiation-reaction-induced transitions of two maximally entangled atoms in noninertial motion, Phys. Rev. D 101 (2020) 025009 [arXiv:2001.00750] [INSPIRE].

[27] H. Cai and Z. Ren, Radiative properties of an inertial multilevel atom in a compactified Minkowski spacetime, Class. Quant. Grav. 36 (2019) 165001 [InSPIRE].

[28] F.N. Lima, R.P.A. Lima and M.L. Lyra, Spontaneous radiation of a two-level system confined in a reflective spherical shell quantum dot, Braz. J. Phys. 49 (2019) 423 [INSPIRE].

[29] X. Liu, Z. Tian, J. Wang and J. Jing, Radiative process of two entanglement atoms in de Sitter spacetime, Phys. Rev. D 97 (2018) 105030 [arXiv:1805.04470] [INSPIRE].

[30] R. de León Ardón, Semiclassical p-branes in hyperbolic space, Class. Quant. Grav. 37 (2020) 237001 [arXiv: 2007.03591] [INSPIRE].

[31] W. Zhou, L. Rizzuto and R. Passante, Vacuum fluctuations and radiation reaction contributions to the resonance dipole-dipole interaction between two atoms near a reflecting boundary, Phys. Rev. A 97 (2018) 042503 [arXiv:1711.08249] [INSPIRE].

[32] G. Menezes, Radiative processes of two entangled atoms outside a Schwarzschild black hole, Phys. Rev. D 94 (2016) 105008 [arXiv: 1512.03636] [INSPIRE].

[33] G. Flores-Hidalgo, M. Rojas and O. Rojas, Entanglement of a two-atom system driven by the quantum vacuum in arbitrary cavity size, arXiv:1511.01416 [INSPIRE].

[34] G. Menezes and N.F. Svaiter, Vacuum fluctuations and radiation reaction in radiative processes of entangled states, Phys. Rev. A 92 (2015) 062131 [arXiv:1508.04513] [INSPIRE].

[35] W. Zhou, R. Passante and L. Rizzuto, Resonance interaction energy between two accelerated identical atoms in a coaccelerated frame and the Unruh effect, Phys. Rev. D 94 (2016) 105025 [arXiv: 1609.06931] [INSPIRE].

[36] W.G. Unruh, Notes on black hole evaporation, Phys. Rev. D 14 (1976) 870 [InSPIRE].

[37] W.G. Unruh and R.M. Wald, What happens when an accelerating observer detects a Rindler particle, Phys. Rev. D 29 (1984) 1047 [InSPIRE].

[38] M.O. Scully, S. Fulling, D. Lee, D.N. Page, W. Schleich and A. Svidzinsky, Quantum optics approach to radiation from atoms falling into a black hole, Proc. Nat. Acad. Sci. 115 (2018) 8131 [arXiv: 1709.00481] [INSPIRE].

[39] K. Chakraborty and B.R. Majhi, Detector response along null geodesics in black hole spacetimes and in a Friedmann-Lemaître-Robertson-Walker universe, Phys. Rev. D 100 (2019) 045004 [arXiv: 1905.10554] [INSPIRE].

[40] B.R. Majhi, Are non-vacuum states much relevant for retrieving shock wave memory of spacetime?, Phys. Lett. B 808 (2020) 135640 [arXiv:2006.04486] [INSPIRE]. 
[41] G. Compère, J. Long and M. Riegler, Invariance of Unruh and Hawking radiation under matter-induced supertranslations, JHEP 05 (2019) 053 [arXiv:1903.01812] [INSPIRE].

[42] M.B. Plenio, S.F. Huelga, A. Beige and P.L. Knight, Cavity loss induced generation of entangled atoms, Phys. Rev. A 59 (1999) 2468 [quant-ph/9811003] [INSPIRE].

[43] Z. Ficek and R. Tanaś, Entanglement induced by spontaneous emission in spatially extended two-atom systems, J. Mod. Opt. 50 (2003) 2765.

[44] R. Tanaś and Z. Ficek, Entangling two atoms via spontaneous emission, J. Opt. B 6 (2004) S90.

[45] S.S. Costa and G.E.A. Matsas, Background thermal contributions in testing the Unruh effect, Phys. Rev. D 52 (1995) 3466 [gr-qc/9412030] [INSPIRE].

[46] S. Kolekar and T. Padmanabhan, Quantum field theory in the Rindler-Rindler spacetime, Phys. Rev. D 89 (2014) 064055 [arXiv:1309.4424] [INSPIRE].

[47] L. Hodgkinson, J. Louko and A.C. Ottewill, Static detectors and circular-geodesic detectors on the Schwarzschild black hole, Phys. Rev. D 89 (2014) 104002 [arXiv:1401.2667] [INSPIRE].

[48] W.G. Brenna, R.B. Mann and E. Martin-Martinez, Anti-Unruh phenomena, Phys. Lett. B 757 (2016) 307 [arXiv: 1504.02468] [INSPIRE].

[49] L.J. Garay, E. Martin-Martinez and J. de Ramon, Thermalization of particle detectors: the Unruh effect and its reverse, Phys. Rev. D 94 (2016) 104048 [arXiv: 1607.05287] [InSPIRE].

[50] S. Kolekar, Uniformly accelerated observer in a thermal bath, Phys. Rev. D 89 (2014) 044036 [arXiv: 1309.3261] [INSPIRE].

[51] S. Kolekar and T. Padmanabhan, Indistinguishability of thermal and quantum fluctuations, Class. Quant. Grav. 32 (2015) 202001 [arXiv: 1308.6289] [INSPIRE].

[52] A. Adhikari, K. Bhattacharya, C. Chowdhury and B.R. Majhi, Fluctuation-dissipation relation in accelerated frames, Phys. Rev. D 97 (2018) 045003 [arXiv: 1707.01333] [INSPIRE].

[53] A. Das, S. Dalui, C. Chowdhury and B.R. Majhi, Conformal vacuum and the fluctuation-dissipation theorem in a de Sitter universe and black hole spacetimes, Phys. Rev. D 100 (2019) 085002 [arXiv: 1902.03735] [INSPIRE].

[54] C. Chowdhury, S. Das, S. Dalui and B.R. Majhi, How robust is the indistinguishability between quantum fluctuation seen from noninertial frame and real thermal bath, Phys. Rev. D 99 (2019) 045021 [arXiv: 1902.06900] [INSPIRE].

[55] A.P.C.M. Lima, G. Alencar and R.R. Landim, Asymptotic states of accelerated qubits in nonzero background temperature, Phys. Rev. D 101 (2020) 125008 [arXiv:2002.02020] [INSPIRE].

[56] R. Banerjee and B.R. Majhi, Fluctuation-dissipation relation from anomalous stress tensor and Hawking effect, Eur. Phys. J. C 80 (2020) 435 [arXiv: 1909.03760] [InSPIRE].

[57] R.H. Dicke, Coherence in spontaneous radiation processes, Phys. Rev. 93 (1954) 99 [INSPIRE].

[58] M. Mijic, Green functions and thermal nature of black holes, in Belgrade workshop (Danube 93), (1993) [hep-th/9311030] [INSPIRE].

[59] H.A. Weldon, Thermal Green functions in coordinate space for massless particles of any spin, Phys. Rev. D 62 (2000) 056010 [hep-ph/0007138] [INSPIRE]. 
[60] L.C.B. Crispino, A. Higuchi and G.E.A. Matsas, The Unruh effect and its applications, Rev. Mod. Phys. 80 (2008) 787 [arXiv:0710.5373] [InSPIRE].

[61] N.D. Birrell and P.C.W. Davies, Quantum fields in curved space, Cambridge Univ. Press, Cambridge, U.K. (1984) [INSPIRE].

[62] S. Carroll, Spacetime and geometry. An introduction to general relativity, Addison Wesley, San Francisco, CA, U.S.A. (2004).

[63] A. Higuchi, S. Iso, K. Ueda and K. Yamamoto, Entanglement of the vacuum between left, right, future, and past: the origin of entanglement-induced quantum radiation, Phys. Rev. D 96 (2017) 083531 [arXiv: 1709.05757] [INSPIRE].

[64] T. Padmanabhan, Gravitation: foundations and frontiers, first edition, Cambridge University Press, Cambridge, U.K. (2009). 